\title{
ANÁLISE DISCRIMINANTE PARA SÉRIES TEMPORAIS
}

Séraio Mikio Kovama

DISSERTAÇÃO APRESENTADA

$\mathrm{AO}$

INSTITUTO DE MATEMÁTICA E ESTATÍSTICA

DA UNIVERSIDADE DE SÃO PAULO

PARA OBTENÇÃO DO GRAU DE MESTRE

EM ESTATISTICA

Área de Concentração: Estatística

Orientadora: Prof. Dra. Clélia Maria de Castro Toloi

Este trabalho foi financiado pelo $\mathrm{CNPQ}$ 
Este exemplar corresponde a redação

da dissertação devidamente corrigida

e apresentado por Sérgio Mikio Koyama

e aprovado pela Comissão Julgadora

São Paulo, 22 de maio de 1997

Banca Examinadora:

- Profa. Dra. Clélia Maria de Castro Toloi - IME - USP

- Prof. Dr. Pedro Morettin - IME - USP

- Prof. Dr. Luiz Kodi Hotta - IMECC - Unicamp 


\section{Agradecimentos}

Em verdade, se necessário fosse colocar aqui o nome de todas as pessoas que ajudaram para que este trabalho fosse realizado, com certeza esta seção seria maior que o próprio corpo do mesmo. Desta forma, gostaria apenas de ressaltar alguns nomes que diretamente contribuíram para a confecção desta dissertação, em particular:

- a Profa. Dra. Clélia Maria de Castro Toloi, paciente orientadora, conselheira e amiga que apesar do longo tempo de espera e sufoco da última instância, não desistiu de mim. A ela sou grato, não só pela paciência e dedicação que me incentivou e transmitiu seus conhecimentos, mas também pela minha atual profissão, na qual teve participação decisiva em meu primeiro ano de faculdade.

- ao Prof. Dr. Júlio da Motta Singer, orientador de minha esposa, pela preocupação e apoio constantes.

- ao Prof. Dr. Denis Donaire, amigo e professor, que por tantas vezes incentivou para que eu pudesse concluir este mestrado, e pela concessão de um dia por semana dedicado a realização deste trabalho.

- ao Doutor Francisco, da Escola Paulista de Medicina, pelo auxílio imprescindível na indicação de colegas que pudessem colaborar no fornecimento de dados para o estudo prático. 
- ao Doutor Carlos Campos, do Setor de Investigação e Tratamento de Epilepsias da Escola Paulista de Medicina, pelo fornecimento dos dados para o estudo prático.

- à Trevisan Consultores, em especial ao Diretor da área de Avaliação Econômico-Financeira, sr. Luiz Nelson, pela concessão de afastamento para que pudesse desenvolver este trabalho.

- ao sr. Adriano Mandelli, Leila, Shirley e Eliana, membros de minha equipe, que mantiveram o departamento em pleno funcionamento nos momentos difíceis da confecção desta dissertação.

- aos amigos da Unitrabalho, em especial ao Prof.Dr. Newton e Prof. Dr. Francisco da UFSCar e a Profa. Marisa, pelo apoio e compreensão às minhas ausências, devido à necessidade de conclusão deste trabalho.

- à Faculdade Capital, em especial ao Prof. Cabral, Cidinha, Prof. Milton Farina, Profa. Chang Chiann e Prof. Wilson Furquim.

- ao Prof. Dr. Antônio Carlos e Prof. Dr. Rinaldo pelo apoio e torcida no término deste trabalho.

- à minha mãe e aos pais de minha esposa pelo apoio, compreensão nos momentos mais difíceis.

- à Marcos e Helena pelo apoio logístico.

- ao meu irmão Ricardo e sua família. 


\section{Resumo}

No presente trabalho apresentamos uma extensão da técnica de análise discriminante para o caso de séries temporais, tanto no domínio do tempo como da freqüência. Foram averiguados o desempenho, no domínio do tempo, de dois modelos: a partir da média dos parâmetros para obtenção do modelo de cada grupo e através da utilização da mediana. No domínio da frequência, comparamos

os estimadores: periodograma, periodograma suavisado e estimador espectral auto-regressivo. Para tanto, realizamos simulações e o estudo de um caso prático de necessidade de discriminação de padrões de eletroencéfalogramas de indivíduos normais e epiléticos. Os resultados apontam para um melhor desempenho do estimador no domínio do tempo utilizando-se a mediana, para os casos de modelos mais simples (sem muitas periodicidades envolvidas), sendo nos casos mais complexos (várias periodicidades) recomendado o modelo baseado apenas no periodograma. 


\section{ABSTRACT}

In this study we present an extension of discriminant analysis techniques to the time series cases in the time and frequency domain. In the time domain, the perform of two models has been checked, one using mean and the other using median of the parameters to obtain the model for each group. In the frequency domain, we compared the folowing estimators: periodogram, smoothed periodogram and spectral autoregressive estimator. In order to do that, we performed simulations and the study of a pratical case where we had the need to discriminated the pattern of electroencephalogram of normal and epileptic persons. In the time domain, the results indicate the median as a good estimator for the simple models case (without many periodicities involved) and in the case with many periodicities involved, we obtained good results using models based in the periodogram. 


\section{Índice}

Capítulo 1 - Introdução 1

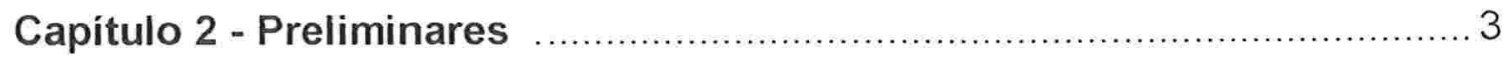

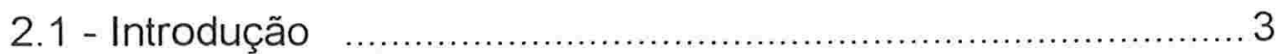

2.2 - Análise de Séries Temporais - Definições …………………... 4

2.3 - Análise Discriminante - Definições ......................................... 21

Capítulo 3 - Análise Discriminante em Séries Temporais .......................... 25

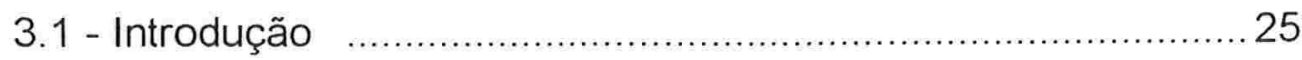

3.2 - Análise Discriminante no Domínio do Tempo …………....... 28

3.3 - Análise Discriminante no Domínio da Frequência ................. 32

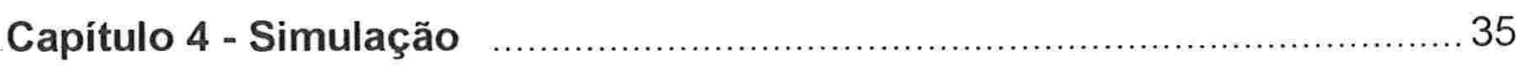

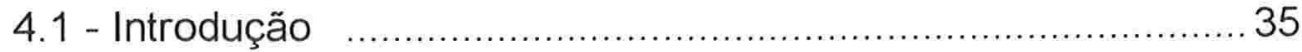

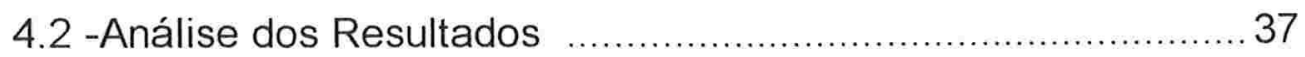

Capítulo 5 - Aplicação a Dados de Eletroencéfalogramas ……….............5 50

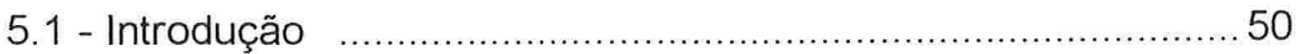

5.2 - Análise Discriminante no Domínio do Tempo ......................56

5.3 - Análise Discriminante no Domínio da Frequência ................65

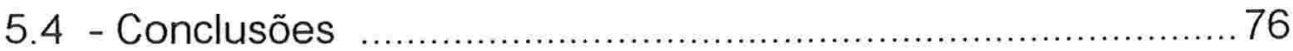

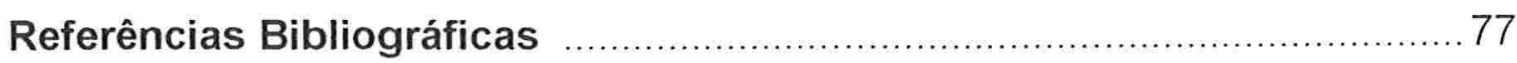

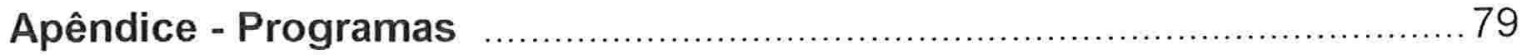

A.1 - Análise Discriminante no Domínio do Tempo ......................79 
A.2 - Análise Discriminante no Domínio da Frequência

Periodograma/ Periodograma Suavizado 83

A.3 - Análise Discriminante no Domínio da Frequência

Estimador Espectral Auto-regressiva. 86 


\section{Capítulo 1}

\section{Introdução}

A análise discriminante, ou análise classificatória, pode ser entendida como uma técnica para a classificação de uma nova observação em relação a grupos previamente existentes, dentre os quais temos interesse especial em alguns deles. Assim, a análise discriminatória tem como objetivo básico a alocação um novo indivíduo dentro de um grupo, a partir de um conjunto de variáveis que servirão para indicar os motivos das diferença entre os mesmos.

O presente trabalho tem por objetivo estudar a técnica de análise discriminante em séries temporais sob dois enfoques: no domínio do tempo e no domínio da frequência. No domínio do tempo, a partir do critério AIC de Akaike, determinamos modelos auto-regressivos comuns para cada grupo visando reduzirmos o número de parâmetros a serem estimados, e calculamos os estimadores dos mesmos, a partir da média ou da mediana dos modelos de cada série componente de cada grupo. Posteriormente a obtenção dos modelos, utilizando-se o critério de minimização do custo esperado de má classificação, obtemos a função discriminante de forma análoga à análise discriminante clássica.

No domínio da frequência, em virtude das propriedades assintóticas da transformada discreta de Fourier, o processo de determinação da função de verossimilhança torna-se simples e, por conseqüência, a função discriminante. Porém, em virtude da existência de diferentes estimadores espectrais, faz-se 
necessário a verificação do desempenho dos mesmos, a saber: periodograma, periodograma suavizado e estimador espectral auto-regressivo.

Desta forma, para atingirmos tal intento, subdividimos o presente trabalho da seguinte forma:

- No capítulo 2, apresentaremos algum resultados e definições básicas (referente tanto às técnicas de séries temporais como às da análise discriminante), de modo a padronizar a notação utilizada;

- No capítulo 3, discorreremos a respeito da técnica de análise discriminante no contexto de séries temporais;

- No capítulo 4, apresentamos os resultados de uma simulação realizada em 12 conjuntos de 220 séries cada, subdivididas em dois grupos;

- No capítulo 5, analisamos um conjunto de séries de eletroencefalogramas de pessoas normais e epiléticas. 


\section{Capítulo 2}

\section{Preliminares}

\subsection{Introdução}

Este capítulo tem por objetivo fornecer as informações básicas para o melhor entendimento da metodologia a ser desenvolvida no transcorrer deste trabalho.

Tendo em vista que, para a realização da análise discriminante em séries temporais será imprescindivel, no domínio do tempo, a imposição de um modelo, abordaremos brevemente os modelos de Box - Jenkins e, no domínio da freqüência, apresentaremos a transformada de Fourier, o periodograma, o periodograma suavizado e o estimador espectral auto-regressivo. Tais tópicos são justificados devido a necessidade de verificarmos a possibilidade da utilização das metodologias de reconhecimento de padrões, tanto no domínio do tempo, onde poderemos a partir de modelos auto-regressivos, reduzir o número de parâmetros a serem estimados, como no do domínio da freqüência, onde a independência assintótica das componentes do periodograma facilitam a determinação dos padrões de cada grupo formado. Além disso, a existência de estimadores espectrais auto-regressivos, os quais apresentam um comportamento mais suavizado e com melhor definição do que os obtidos a partir dos métodos tradicionais, mediante ao uso das técnicas não-paramétricas,ver Ara (1982), serão utilizados com o objetivo de comparar as performances destes dois estimadores no reconhecimento de padrões. 
Inicialmente, apresentaremos algumas definições de modo a padronizar nossa notação e a facilitar a compreensão de algumas propriedades e motivos que nos levaram a utilização das técnicas empregadas.

\subsection{Análise de Séries Temporais - Definições}

Definição 2.2.1: Um processo estocástico $X=\{X(t, \theta) ; t \in T, \quad \theta \in \Omega\}$ é uma coleção de variáveis aleatórias definidas sobre um espaço de probabilidade $(\Omega$, $F, P)$ e indexadas pelos elementos de um conjunto de parâmetros T. Por exemplo, se $T=[a, b] \in \mathfrak{R}$, diremos que $X(t, \theta)$ é um processo estocástico com parâmetro contínuo, ao passo que, se $T \subset Z$, teremos um processo estocástico com parâmetro discreto, também denominado seqüência aleatória.

Definição 2.2.2: Sendo $X(t, \theta)$ uma função de duas variáveis, $t \in T$ e $\theta \in \Omega$, para $t$ fixo, a função resultante $X(., \theta)$ é mensurável em $F$, para $\theta$ fixo, obtemos uma função $X(., \theta): T \rightarrow \mathfrak{R}$ que é chamada trajetória, realização, função amostral do processo ou simplesmente série temporal.

Portanto podemos dizer que uma série temporal é um conjunto de observações ordenadas segundo uma seqüência natural. Cabe ressaltar que apesar da titulação "temporal", é importante lembrar que a variável tempo, embora a mais usual, não é a única indexadora possivel desta série, sendo a existência de uma "lei" de formação (ou a inter-relação) existente entre as diversas observações desta seqüência o fator determinante, que diferencia este conjunto 
de valores de outros e que impossibilita, na maioria dos casos, a utilização das técnicas tradicionais de análise. Visando a simplificação da notação, a partir deste momento, denotaremos uma série temporal, ao invés de $X(t, \theta)$, simplesmente por $X(t)$.

Definição 2.2.3: Dado uma série temporal $X(t), t \in T$, definimos suas distribuições finito-dimensionais por

$$
\begin{aligned}
& F\left(x_{1}, x_{2}, \ldots, x_{n} ; t_{1}, t_{2}, \ldots, t_{n}\right)=P\left\{X\left(t_{1}\right) \leq x_{1}, \ldots, X\left(t_{n}\right) \leq x_{n}\right\} ; \\
& n \geq 1 ; t_{1}, t_{2}, \ldots, t_{n} \in T \text { e } x_{1}, x_{2}, \ldots, x_{n} \in X
\end{aligned}
$$

Definição 2.2.4 As funções média, variância e (auto)covariância de uma série temporal $X(t)$ são dadas por:

a) $\quad E\{X(t)\}=\int_{-\infty}^{+\infty} X \mathrm{dF}(\mathrm{X} ; \mathrm{t})=\mu(t)$

b) $\quad \operatorname{Var}\{X(t)\}=\int_{-\infty}^{+\infty}[x-\mu(t)]^{2} d F(x ; t)=\gamma_{x, x}(t, t)$

c) $\operatorname{Cov}\left\{X\left(t_{1}\right), X\left(t_{2}\right)\right\}=\int_{-\infty}^{+\infty} \int_{-\infty}^{+\infty}\left[x\left(t_{1}\right)-\mu\left(t_{1}\right)\right]\left[x\left(t_{2}\right)-\mu\left(t_{2}\right)\right] d F\left(x\left(t_{1}\right), x\left(t_{2}\right) ; t_{1}, t_{2}\right)$

$$
=\gamma_{x, x}\left(t_{1}, t_{2}\right),
$$


das quais podemos derivar as funções de autocorrelação e correlação cruzada, ver Brillinger (1981)

Definição 2.2.5: Sejam $X(t)$ e $Y(t), t \in T$, duas séries temporais. As funções de autocorrelação e correlação cruzada são dadas por:

a) $\quad \rho_{x, x}\left(t_{1}, t_{2}\right)=\frac{\gamma_{x . x}\left(t_{1}, t_{2}\right)}{\sqrt{\gamma_{x . x}\left(t_{1}, t_{1}\right)} \sqrt{\gamma_{x . x}\left(t_{2}, t_{2}\right)}}$,

b) $\quad \rho_{x, y}\left(t_{1}, t_{2}\right)=\frac{\gamma_{x, y}\left(t_{1}, t_{2}\right)}{\sqrt{\gamma_{x . x}\left(t_{1}, t_{1}\right)} \sqrt{\gamma_{y, y}\left(t_{2}, t_{2}\right)}}$.

onde:

$\gamma_{x, y}\left(t_{1}, t_{2}\right)=\int_{-\infty}^{+\infty} \int_{-\infty}^{+\infty}\left[x\left(t_{1}\right)-\mu_{x}\left(t_{1}\right)\right]\left[y\left(t_{2}\right)-\mu_{y}\left(t_{2}\right)\right] d F\left(x\left(t_{1}\right), y\left(t_{2}\right) ; t_{1}, t_{2}\right)$

As funções de autocovariância e de autocorrelação dadas por (2. 4) e (2.5), têm as seguintes propriedades:
a) $\gamma_{x, x}(0)=\operatorname{Var}\{X(t)\}>0$;
b) $\gamma_{x, x}(t, t+\Delta t)=\gamma_{x, x}(t+\Delta t, t)$;
c) $\left|\gamma_{x, x}(t+\Delta t, t)\right| \leq \gamma_{x, x}(0)$;
d) $\rho_{x, x}(t, t)=\rho_{x, x}(0)=1$;
e) $\rho_{x, x}(t+\Delta t, t)=\rho_{x, x}(\Delta t)$;
f) $\left|\rho_{x_{1} x}(\Delta t)\right| \leq 1$. 
Definição 2.2.6:(a) Seja $\left(X_{1}, \ldots, X_{r}\right)$ uma variável aleatória r-dimensional com $E\left|X_{j}\right|^{r}<\infty, j=1, \ldots, r$, onde $X_{j}$ são reais ou complexas. O cumulante conjunto de ordem r é dado por:

$$
\operatorname{Cum}\left(X_{1}, \ldots, X_{r}\right)=\sum_{v}(-1)^{p-1}(p-1) !\left(E \prod_{j \in v_{i}} X_{j}\right) \ldots\left(E \prod_{j \in v_{p}} X_{j}\right)
$$

onde a soma é sobre todas as partições $v=\left(v_{1}, \ldots, v_{p}\right)$ dos inteiros $(1, \ldots, r)$.

(b) Seja uma série temporal $\{X(t), t \in T\}$ satisfazendo $E|X(t)|^{k}<\infty$, então:

$$
c_{x}\left(t_{1}, \ldots, t_{r}\right)=\operatorname{Cum}\left(X\left(t_{1}\right), \ldots, X\left(t_{r}\right)\right)
$$

é denominada função cumulante conjunta de ordem $r$ da série $X(t)$.

As integrais correspondentes as equações 2.2, 2.3 e 2.4, nem sempre existem, sendo necessárias, portanto, algumas condições que garantam sua existência. Desta forma, definiremos condições que restrinjam o conjunto de séries tal que estes valores existam.

Definição 2.2.7: Uma série $\{X(t), t \in T\}$ é estritamente estacionária se todas as distribuições finito-dimensionais forem invariantes sob uma translação comum em t. Assim, teremos:

$$
\begin{aligned}
& F\left(x_{1}, x_{2}, \ldots, x_{n} ; t_{1}, t_{2}, \ldots, t_{n}\right)=F\left(x_{1}, x_{2}, \ldots, x_{n} ; t_{1}+\Delta t, t_{2}+\Delta t, \ldots, t_{n}+\Delta t\right) \\
& \text { quaisquer } t_{1}, t_{2}, \ldots, t_{n}, \Delta t \in T
\end{aligned}
$$


de onde segue-se que todos os momentos de ordem $n$ dependem apenas das diferenças $t_{j}-t_{1}$, o qual devido a extensão, torna-se difícil de ser verificada. Restringindo-nos apenas aos controle dos momentos de 1a. e 2a. ordem, construimos um segundo conjunto de séries com restrições mais brandas.

Definição 2.2.8: Uma série temporal $\{X(t), t \in T\}$ é estacionária de segunda ordem ou fracamente estacionária se:

a) $\quad E\{X(t)\}=\mu(t)=\mu, \forall t \in T$,

b) $\quad E\left\{X^{2}(t)\right\}<\infty, \forall t \in T$,

c) $\operatorname{Cov}\{X(t+\Delta t), X(t)\}=\gamma_{x, x}(t+\Delta t, t)=\gamma_{x, x}(\Delta t)$.

Para maiores informações, ver Priestley (1981).

Desta forma, notamos a existência de duas classes de processos estacionários, uma mais forte, restritiva e uma outra mais branda, fraca. Entretanto, apesar das designações "processo estritamente estacionário ou estacionariedade forte"e "processo fracamente estacionário ou estacionário de 2a. ordem", tais classes não apresentam as qualidades de "contido"e "contém" e, sim, a da "intersecção", sendo o caso mais interessante o que se segue:

Definição 2.2.9: Um processo estocástico $\{X(t), t \in T\}$ diz-se Gaussiano se para qualquer conjunto $t_{1}, \ldots t_{n} \in T$, as variáveis aleatórias $X\left(t_{1}\right), \ldots, X\left(t_{n}\right)$ possuem distribuição normal n-variada. Se um processo for Gaussiano (ou Normal) ele será 
determinado pelas médias e covariâncias, e em particular, se for estacionário de 2a. ordem, ele será também estritamente estacionário.

Devido a nossa maior preocupação com o conjunto de séries estacionárias de 2a. ordem, em virtude da maior facilidade de sua comprovação (enquadramento), iremos nos referir a esta classe, de maneira simplificada, apenas como séries estacionárias.

Em nosso estudo, trabalharemos com as séries denominadas estacionárias e não estacionárias homogêneas, que se transformam em estacionária mediante a aplicação de diferenças sucessivas.

Definição 2.2.10: Seja $\{X(t), t \in T\}$ uma série temporal estacionária. A função de autocorrelação parcial $\left(\phi_{k k}\right)$ corresponde à correlação entre $X(t)$ e $X(t+k)$ eliminando a dependência linear das variáveis intervenientes $\mathrm{X}(\mathrm{t}+1), \mathrm{X}(\mathrm{t}+2), \ldots$, $X(t+k-1)$. O cálculo de $\phi_{k k}$ conforme Wei (1990), pode ser obtido como o coeficiente da variável $X(t)$ num modelo de regressão linear com variável dependente $X(t+k)$ e variáveis independentes $X(t+k-1), X(t+k-2), \ldots, X(t)$. Assim a autocorrelação parcial é dada por: 


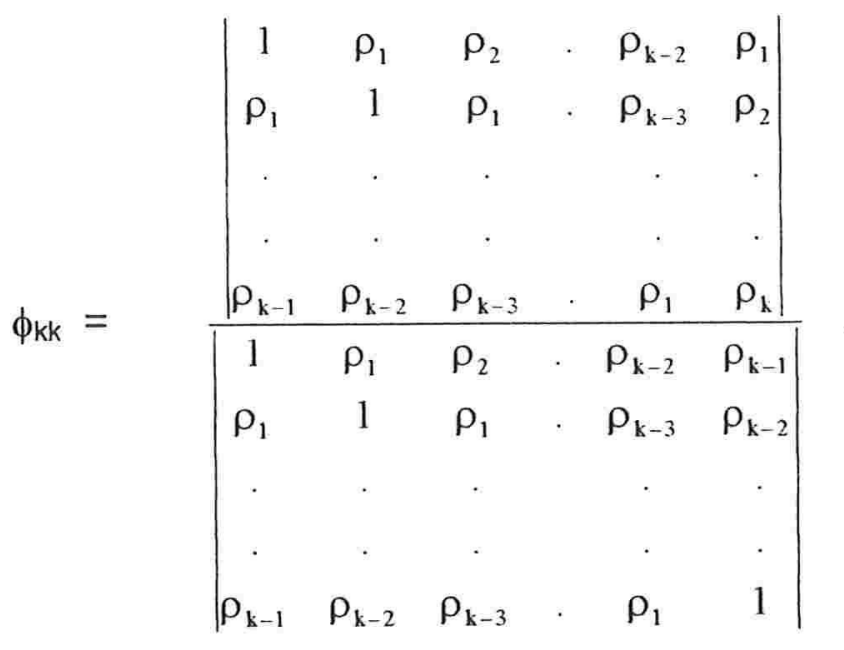

Definição 2.2.11: Um processo $\{a(t), t \in T\}$ é chamado de ruído branco se a ele corresponder uma sequência de variáveis aleatórias com as seguintes propriedades:
a) $E(a(t))=\mu_{a}\left(\right.$ usualmente $\left.\mu_{a}=0\right)$,
b) $\operatorname{Var}(a(t))=\sigma_{a}^{2}$,
c) $\gamma_{a, a}(k)=\operatorname{Cov}(a(t+k), a(t))=0, \forall k \neq 0$.

Definição 2.2.12: Seja $\{a(t), t \in T\}$ um ruído branco com média 0 e variância $\sigma_{a}^{2}$ e sejam $\phi_{1}, \phi_{2}, \ldots, \phi_{p}, \theta_{1}, \ldots, \theta_{\mathrm{q}}$ números reais constantes tais que $\phi_{\mathrm{p}} \neq 0$ e $\theta_{\mathrm{q}} \neq 0$. Então dizemos que $X(t)$ é um processo auto-regressivo - médias móveis de ordem $p, q, \operatorname{ARMA}(p, q)$, se

$$
\begin{aligned}
& \widetilde{X}(t)=\phi_{1} \widetilde{X}(t-1)+\phi_{2} \widetilde{X}(t-2)+\ldots+\phi_{p} \widetilde{X}(t-p)+a_{t}-\theta_{1} a(t-1)-\ldots-\theta_{q} a(t-q) \\
& t \in T \text {, onde } \widetilde{X}(t)=X(t)-\mu
\end{aligned}
$$


Definição 2.2.13: O operador translação para o passado, denotado por B é tal que:

$$
B^{k} \bar{X}(t)=\bar{X}(t-k)
$$

Utilizando (2.15) podemos reescrever (2.14) na forma reduzida:

$$
\phi(B) \bar{X}(t)=\theta(B) a(t)
$$

onde $\phi(B)=1-\phi_{1} B-\phi_{2} B^{2}-\ldots-\phi_{p} B^{p}$ e $\theta(B)=1-\theta_{1} B-\theta_{2} B^{2}-\ldots-\theta_{q} B^{4}$ são denominados operadores auto-regressivo de ordem p e de média móvel de ordem $q$, respectivamente. No caso em que $q=0$, temos um processo denominado processo auto-regressivo, e se $p=0$, denominamos como um processo de médias móveis.

Definição 2.2.14: Um processo auto-regressivo será estacionário se a série $\phi(\mathrm{B})$ convergir para $|\mathrm{B}|<1$. Para maiores detalhes, ver Box, Jenkins e Reinsel (1994)

A análise de Fourier, análise harmônica ou análise espectral, tem como objetivo básico escrever uma série temporal $X(t)$ como uma soma de componentes senoidais (senos e cossenos), buscando assim um maior entendimento dos dados e do sistema no qual foram produzidos.

Definição 2.2.15: Sejam $\phi_{k}(t)$ e $\phi_{j}(t)$, funções complexas definidas no domínio $T$, discreto, subconjunto dos reais. Diremos serem funções ortogonais se 


$$
\sum_{i-1} \phi_{k}(t) \bar{\phi}_{j}(t) \quad \begin{cases}=0 & k \neq j \\ \neq 0 & k=j\end{cases}
$$

onde $\bar{\phi}_{\mathrm{j}}(\mathrm{t})$ representa o conjugado de $\phi_{\mathrm{j}}(\mathrm{t})$.

$$
\text { O sistema }\left\{\operatorname{sen}\left(\frac{2 \pi \mathrm{kt}}{\mathrm{T}}\right), \cos \left(\frac{2 \pi \mathrm{kt}}{\mathrm{T}}\right), \mathrm{k}=0,1, \ldots,\left[\frac{\mathrm{T}}{2}\right]\right\} \text { representa uma }
$$

sequência de funções ortogonais, cujos produtos cruzados apresentam, conforme Wei (1990) e Priestley(1981), as seguintes propriedades:

a)

$$
\sum_{i=1}^{T} \cos \left(\frac{2 \pi k t}{T}\right) \cos \left(\frac{2 \pi j t}{T}\right)= \begin{cases}T & k=j=0, \frac{T}{2} \text { (para } T \text { par) } \\ \frac{T}{2} & k=j \neq 0, \frac{T}{2} \text { (para } T \text { par) } \\ 0 & k \neq j\end{cases}
$$

b)

$$
\sum_{\mathrm{i}=1}^{\mathrm{T}} \operatorname{sen}\left(\frac{2 \pi \mathrm{kt}}{\mathrm{T}}\right) \operatorname{sen}\left(\frac{2 \pi \mathrm{jt}}{\mathrm{T}}\right)= \begin{cases}\mathrm{T} & \mathrm{k}=\mathrm{j}=0, \frac{\mathrm{T}}{2} \text { (para } \mathrm{T} \text { par) } \\ \frac{\mathrm{T}}{2} & \mathrm{k}=\mathrm{j} \neq 0, \frac{\mathrm{T}}{2} \text { (para } \mathrm{T} \text { par) } \\ 0 & \mathrm{k} \neq \mathrm{j}\end{cases}
$$

c) $\quad \sum_{i=1}^{T} \operatorname{sen}\left(\frac{2 \pi k t}{T}\right) \cos \left(\frac{2 \pi j t}{T}\right)=0 \quad \forall k, j$

Definição 2.2.16 Seja $\{X(t), t=0,1,2, \ldots, T-1\}$ uma série temporal observada. $A$ série de Fourier para $X(t)$, segundo Bloomfield (1976) é dada por : 


$$
X(t)=\sum_{i=1}^{\left|\frac{T}{2}\right|}\left(A_{i} \cos ()_{i} t+B_{i} \operatorname{sen}()_{i} t\right), t=0,1, \ldots, T-1
$$

onde $\omega_{\mathrm{i}}=\frac{2 \pi \mathrm{i}}{\mathrm{T}}$ são as frequências de Fourier medidos em radianos por unidade de tempo e $A_{1}$ e $B_{1}$ são os coeficientes de Fourier, que podem ser obtidos utilizando-se as propriedades ortogonais (2.18) - (2.20).

Definição 2.2.17 A Transformada Discreta de Fourier $(d(k))$ de uma série temporal estacionária $\{X(t), t=0,1,2, \ldots, T-1\}$ é dada por :

$$
d_{x}(k)=(2 \pi T)^{-1 / 2} \sum_{i=0}^{T-1} X(t) \exp \left(-i \omega_{k} t\right), \quad k=0,1,2, \ldots, T-1
$$

onde $\omega_{k}=\frac{2 \pi k}{T}$.

Invertendo-se (2.22), temos a transformada inversa dada por:

$$
X(t)=(2 \pi T)^{-1 / 2} \sum_{k=0}^{T-1} d_{x}(k) \exp \left(i \omega_{k} t\right), t=0,1, \ldots, T-1 .
$$

Ainda com base em (2.22), nota-se que $d_{x}(k+T)=d_{x}(k)$, indicando que $d_{x}(k)$ apresenta uma periodicidade igual a $2 \pi\left(\omega_{\mathrm{k}+\mathrm{T}}=\frac{2 \pi \mathrm{k}}{\mathrm{T}}+2 \pi\right)$.

Definição 2.2.18: Seja $\left\{X_{t}, t=0, \pm 1, \pm 2, \ldots\right\}$ uma série temporal estacionária com 


$$
\sum_{\mathrm{m}=-\gamma_{x}}^{+}\left|\gamma_{x}(\mathrm{~m})\right|<x
$$

a funçã̇o densidade espectral de $X(t)$, na frequência (1. é dada por:

$$
f_{x x}(\omega)=\sum_{m=-x}^{r} \gamma_{x x}(m) \exp (-i \omega m),-x<\omega<-s
$$

Teorema 2.1: Seja $\left\{X_{t}, t=0,1,2, \ldots, T-1\right\}$ uma série temporal estacionária e $\left\{d_{x}(k)=d_{x}^{c}(k)+i d_{x}^{s}(k), k=0,1, \ldots, T / 2\right\}$ sua transformada de Fourier, onde $d_{x}^{c}(k)=T^{-1 / 2} \sum_{t=0}^{T-1} X(t) \cos \left(\omega_{k} t\right) e d_{x}^{s}(k)=T^{1 / 2} \sum_{t=0}^{T-1} X(t) \operatorname{sen}\left(\omega_{k} t\right)$. Supondo $E(X(t))=\mu e$ $\sum_{m=\infty}^{\infty}|m|\left|\gamma_{x x}(m)\right|<\infty$, temos que:

a) $d_{\mu}(k)=E\left(d_{x}(k)\right)=(2 \pi T)^{-1 / 2} \sum_{t=0}^{T-1} \mu \exp \left(-i \omega_{k} t\right), \forall k=0,1, \ldots, T-1$,

b)

$$
\begin{aligned}
& \operatorname{Cov}\left(d_{x}^{c}(k), d_{x}^{c}(v)\right)= \begin{cases}f_{x x}\left(\omega_{k}\right), & k=v=0, T / 2 \text { (para T par) } \\
f_{x x}\left(\omega_{k}\right)+\varepsilon_{1}, & k=v \neq 0, T / 2 \text { (para T par) } \\
\varepsilon_{1} & , k \neq 1\end{cases} \\
& \operatorname{Cov}\left(d_{x}^{s}(k), d_{x}^{s}(v)\right)= \begin{cases}f_{x x}\left(\omega_{k}\right) & , k=v=0, T / 2 \text { (para T par) } \\
f_{x x}\left(\omega_{k}\right)+\varepsilon_{1}, & k=v \neq 0, T / 2 \text { (para T par) } \\
\varepsilon_{1} & , k \neq 1\end{cases} \\
& \operatorname{Cov}\left(d_{x}^{c}(k), d_{x}^{s}(v)\right)=\varepsilon_{i},
\end{aligned}
$$

onde $\varepsilon_{\mathrm{t}}$ representa um erro limitado por $\left|\varepsilon_{\mathrm{t}}\right| \leq \mathrm{T}^{-1} \sum_{\mathrm{m}=--}^{\prime}|\mathrm{m}| \gamma_{\mathrm{xx}}(\mathrm{m})$. Para maiores detalhes, ver Shumway (1988). 
Do Teorema 2.1 verifica-se que para $\mathrm{k} \neq 0, \mathrm{~T} / 2$ (para $\mathrm{T}$ par):

$$
\operatorname{Cov}(d(k), d(1)) \cong\left\{\begin{array}{ll}
f_{x x}\left(\omega_{k}\right), & k=1 \\
0 & k \neq 1
\end{array} .\right.
$$

Definição 2.2.19: Um vetor $p$-dimensional $\mathbf{X}^{\top}=\left(X_{1}, X_{2}, \ldots, X_{p}\right)$ terá uma distribuição conjunta normal multivariada de dimensão $p$, isto é, $X \sim N_{p}\left(\mu, \Sigma_{x x}\right)$, se e somente se sua função densidade for dada por

$$
f_{x}(X)=(2 \pi)^{-p / 2}\left|\Sigma_{x_{x}}\right|^{-1} \exp \left(-\frac{1}{2}(X-\mu)^{T} \sum_{x_{x}}{ }^{-1}(X-\mu)\right)
$$

onde $E(X)=\mu$ e $\sum_{x x}$ é a matriz de covariância de $\mathbf{X}$.

Definição 2.2.20: Se $Z=X+i Y$ é um vetor de ordem $p \times 1$ com componentes complexos, dizemos que $\mathbf{Z}$ tem distribuição normal complexa multivariada com média $\mu_{z}$ e matriz de covariância $\sum_{z z}$, isto é , $Z \sim N_{p}^{c}\left(\mu_{z}, \sum_{z z}\right)$, onde

$$
\mu_{z}=\left(\begin{array}{l}
\mu_{x} \\
\mu_{y}
\end{array}\right) \text { e } \quad \sum_{z z}=\left[\begin{array}{cc}
\sum_{x x} & -\Sigma_{y y} \\
\sum_{y y} & \sum_{x x}
\end{array}\right]
$$

se e somente se a matriz $(\mathbf{X}, \mathbf{Y})^{\top}$ de componentes reais de ordem $2 p \times 1$ tem distribuição

$$
N_{2 p}\left(\left[\begin{array}{l}
\mu_{s} \\
\mu_{y}
\end{array}\right] ; \frac{1}{2}\left[\begin{array}{cc}
\Sigma_{x x} & -\Sigma_{y y} \\
\Sigma_{y y} & \Sigma_{x x}
\end{array}\right]\right)
$$


onde a matriz $\sum_{z z}$ é uma matriz Hermitiana não negativa definida de dimensão p×p. Maiores detalher, ver Brillinger (1981).

\section{Propriedades da distribuição normal multivariada:}

a) Se $\quad \sum_{z z}$ é uma matriz diagonal, então as componentes de $Z$ são estatisticamente independentes.

b) No caso de $p=1$, se $Z \sim N^{c}{ }_{1}\left(\mu_{z}, \sigma_{z z}\right)$ com $\sigma_{z z} \in R$, então $X$ e $Y$ são independentes com distribuições $X \sim N_{1}\left(\mu_{x}, \sigma_{z z} / 2\right)$ e $Y \sim N_{1}\left(\mu_{y}, \sigma_{z z} / 2\right)$.

Definição 2.2.21: Diremos que uma sequência vetorial $\left\{Z_{t}, t=0,1,2, \ldots\right\}$ é assintoticamente $\quad N_{p}\left(\mu_{t}, \sum_{t t}\right)$, se a sequência $\sum_{t t}^{-1 / 2}\left(Z_{t}-\mu_{t}\right) \stackrel{D}{\rightarrow} N_{p}(0, I)$.

Definição 2.2.22: Uma sequência vetorial $\left\{Z_{t}, t=0,1,2, \ldots\right\}$ é assintoticamente $N_{p}^{c}\left(\mu_{t}, \sum_{t t}\right)$ se a sequência $\sum_{t t}^{-1 / 2}\left(Z_{t}-\mu_{t}\right) \stackrel{D}{\rightarrow} N_{p}^{c}(0, I)$

Teorema 2. 2 : Seja $\quad\{X(t), t=0,1, \ldots, T-1\}$ uma série temporal $e$ $\left\{d_{x}(k), k=0,1, \ldots, T-1\right\}$ sua transformada de Fourier. Então $\left\{d_{x}(k), k=1, \ldots, T-1\right\}$ são aproximadamente $N^{c}{ }_{1}\left(d_{u 1}(k), f_{x x}\left(\omega_{k}\right)\right)$ independentes, onde $d_{u 1}(k)$ é dado por (2.30)

Definição 2.2.23: Seja uma série temporal estacionária $\{X(t), t=0,1,2, \ldots, T-1\}$, $d_{x}(k)=d_{x}^{c}(k)+i d_{w}^{s}(k)$, sua transformada de discreta de Fourier e $f_{x x}(\omega)$ sua função 
densidade espectral. O periodograma, estimador natural para $f_{x x}\left(\omega_{k}\right)$ é definido por:

$$
\hat{f}_{x . x}^{\prime}\left(\omega_{k}\right)=\left|d_{x}(k)\right|^{2}=\left(d_{x}^{c}(k)\right)^{2}+\left(d_{x}^{s}(k)\right)^{2}
$$

onde $\omega_{k}=\frac{2 \pi k}{T}$.

\section{Propriedades do periodograma:}

Se $\{X(t), t=0,1, \ldots, T-1\}$ é uma série temporal estacionária satisfazendo:

$$
\sum_{u_{1}, \ldots, \mathrm{c}_{k-1}=-\infty}^{s}\left|\mathrm{c}_{\mathrm{x}_{1}, \ldots, \mathrm{s}_{k}}\left(\mathrm{u}_{1}, \ldots, \mathrm{u}_{\mathrm{k}-1}\right)\right|<\infty
$$

então:

a) $\hat{\mathrm{f}}_{x . \mathrm{x}}^{1}\left(\omega_{\mathrm{k}}\right), \mathrm{k} \neq 0, \mathrm{~T} / 2$ são assintoticamente independentes com distribuição $\frac{f_{x x}\left(\omega_{k}\right)}{2} \chi_{2}^{2}$ e $f_{x x}\left(\omega_{k}\right) \chi_{2}^{2}$ para $k=0$, T/2, onde $c_{x_{1} \ldots x_{k}}\left(u_{1}, \ldots, u_{k}\right)$ é o cumulante conjunto de ordem $\mathrm{k}$ de $\mathrm{X}_{1}, \ldots, \mathrm{X}_{\mathrm{k}}$.

b) Se $\sum_{m=s c}^{\prime}|m|\left|\gamma_{x x}(m)\right|<\infty$ então:

- $E\left[\hat{f}_{x . x}^{1}\left(\omega_{1}\right)\right]=f_{x x}\left(\omega_{k}\right)+O\left(T^{-1}\right)$ (assintoticamente não viciado)

- $\operatorname{Var}\left[\hat{f}_{x x}^{1}\left(\omega_{k}\right)\right]=f_{x x}^{2}\left(\omega_{k}\right)+O\left(T^{1}\right)$

- $\operatorname{Cov}\left[\hat{\mathrm{f}}_{\mathrm{xx}}^{1}\left(\omega_{k}\right), \hat{\mathrm{f}}_{\mathrm{xx}}^{1}\left(\omega_{1}\right)\right]=\mathrm{O}\left(\mathrm{T}^{1}\right)$ 
ou seja, $\hat{f}_{x . x}^{1}\left(\omega_{k}\right)$ para $k \neq 0$, apesar de serem assintoticamente independentes, não são consistentes, pois suas variâncias não convergem para zero quando $\mathrm{T} \rightarrow \infty$, ver Brillinger (1981).

Definição 2.2.24 Seja $\{X(t), t=0,1, \ldots, T-1\}$ uma série temporal e $f_{x x}\left(\omega_{k}\right)$ o espectro de $X(t)$. Define-se o periodograma suavizado $\left(\hat{f}_{x . x}^{1}\left(\left(_{k}\right)\right)\right.$ como a média dos $L$ valores do periodograma centrado na frequência $\omega_{\mathrm{k}}$

$$
\begin{aligned}
\hat{\mathrm{f}}_{\mathrm{x} . \mathrm{x}}^{\mathrm{L}}\left(\omega_{\mathrm{k}}\right) & =\mathrm{L}^{-1} \sum_{\mathrm{l}=-(\mathrm{L}-1) / 2}^{(\mathrm{L}-1) / 2} \mathrm{~W}\left(\omega_{\mathrm{k}+1}\right) \hat{\mathrm{f}}_{\mathrm{xx}}^{1}\left(\omega_{\mathrm{k}}+\frac{2 \pi \mathrm{l}}{\mathrm{T}}\right)= \\
& =\mathrm{L}^{-1} \sum_{\mathrm{l}=-(\mathrm{L}-1) / 2}^{(\mathrm{L}-1) / 2} \mathrm{~W}\left(\omega_{\mathrm{k}+1}\right)\left|\mathrm{d}_{\mathrm{x}}(\mathrm{k}+1)\right|^{2},
\end{aligned}
$$

onde $W\left(\omega_{k}\right)$ é denominada janela espectral, que corresponde a uma função peso e $L=2 m+1, m=0,1, \ldots$ ou seja $L$ é ímpar.

\section{Propriedades do periodograma suavisado:}

Se $\{X(t), t=0,1, \ldots, T-1\}$ é uma série temporal estacionária satisfazendo (2.35) então

a) $\hat{f}_{x . .}^{L}\left(\omega_{k}\right), k \neq 0, T / 2$ são assintoticamente independentes com distribuição $\frac{f_{x x}\left(\omega_{k}\right)}{2 L} \chi_{2 L}^{2}$ e $\frac{f_{x x}\left(\omega_{k}\right)}{L-1} \chi_{2 L}^{2}$ para $k=0, T / 2$,

b) Se $\sum_{m=\infty}^{\infty}\left|\mathrm{m} \| \gamma_{\mathrm{x}}(\mathrm{m})\right|<\infty$ então:

- $E\left[\hat{f}_{x . x}^{L}\left(\omega_{k}\right)\right]=f_{x x}\left(\omega_{k}\right)+O\left(T^{-1}\right)$ (assintoticamente não viciado), 
- $\operatorname{Var}\left[\hat{f}_{x x}^{L}\left(\omega_{k}\right)\right]=\frac{f_{x . s}^{2}\left(\left(\omega_{h}\right)\right.}{L}+O\left(T^{1}\right)$

- $\operatorname{Cov}\left[\hat{\mathrm{f}}_{\mathrm{xx}}^{\mathrm{l}}\left(\omega_{\mathrm{k}}\right), \hat{\mathrm{f}}_{\mathrm{xx}}^{\mathrm{l} \cdot \mathrm{l}}\left(\omega_{1}\right)\right]=\mathrm{O}\left(\mathrm{T}^{1}\right)$

ou seja, $\hat{\mathrm{f}}_{\mathrm{x..x}}^{\mathrm{L}}\left(\left(_{\mathrm{k}}\right)\right.$ é estimador consistente.

Maiores detalhes, ver Brillinger (1981).

Definição 2.2.25 Seja $\{X(t), t=0,1, \ldots, T-1\}$ uma série temporal e $f_{x x}\left(\omega_{k}\right) 0$ espectro de $X(t)$. Seja $\hat{\phi}_{1}, \hat{\phi}_{2}, \ldots, \hat{\phi}_{\mathrm{p}}$ e $\hat{\sigma}_{a}^{2}$, estimativas de $\phi_{1}, \phi_{2}, \ldots, \phi_{\mathrm{p}}$ e $\sigma_{\mathrm{a}}^{2}$, respectivamente, de um modelo $A R(p)$. O estimador espectral auto-regressivo sugerido por Akaike (1969) e Parzen (1974) é definido por

$$
\hat{f}_{x . x}^{\Lambda}\left(\omega_{h}\right)=\frac{\hat{\sigma}_{3}^{2}}{2 \pi} \cdot \frac{1}{\hat{\Phi}_{p}(\exp (-i \omega)) \hat{\Phi}_{p}(\exp (i \omega))},
$$

onde $\hat{\Phi}_{\mathrm{p}}(\exp (-\mathrm{i} \omega))=1-\hat{\phi}_{1} \exp (-\mathrm{i} \omega)-\quad \ldots-\hat{\phi}_{\mathrm{p}} \exp (-\mathrm{ip} \omega)$

Propriedades do estimador espectral auto-regressivo:

Seja $\{X(t), t=0,1, \ldots, T-1\}$ uma série temporal estacionária representada por um modelo $\operatorname{ARMA}(p, q)$,

$$
\phi(B) \widetilde{X}(t)=\theta(B) a(t)
$$

Reescrevendo $X(t)$ em função apenas de seu passado e do ruido presente, temos

$$
\Pi(B) \bar{X}(t)=a(t)
$$

onde $\Pi(B)=1-\pi_{1} B-\pi_{2} B^{2}-\ldots$ o qual pode ser aproximado por 


$$
\left(1-\pi_{1} B-\pi_{2} B^{2}-\ldots \pi_{M} B^{M}\right) \widetilde{X}(t)=a(t) .
$$

Supondo válidas as seguintes restrições:

- $(\exp (\mathrm{i} \lambda))$ é não nula, $-\pi<\lambda<\pi$

$-E[a(t)]^{4}<\infty, \forall t$

- $O$ valor de $M$ é tal que $\frac{M^{3}}{\pi} \rightarrow 0$ e $M^{1 / 2}\left(\left|\pi_{M+1}\right|+\left|\pi_{M+2}\right|+\ldots\right) \rightarrow 0$

temos que :
a) $\hat{f}_{x x}^{A}\left(\omega_{k}\right) \stackrel{p}{\longrightarrow} f_{x x}\left(\omega_{k}\right)$
b) $\left(\frac{\mathrm{T}}{\mathrm{M}}\right)^{1 / 2}\left[\hat{\mathrm{f}}_{\mathrm{xx}}^{\mathrm{A}}\left(\omega_{\mathrm{k}}\right)-\mathrm{f}_{\mathrm{xx}}\left(\omega_{\mathrm{k}}\right)\right] \sim\left\{\begin{array}{l}N\left(0,2 \mathrm{f}_{\mathrm{xx}}^{2}\left(\omega_{\mathrm{k}}\right)\right), \mathrm{k} \neq 0, \mathrm{~T} / 2 \\ N\left(0,4 \mathrm{f}_{\mathrm{xx}}^{2}\left(\omega_{\mathrm{k}}\right)\right), \mathrm{k}=0, \mathrm{~T} / 2\end{array}\right.$

Assim, $\hat{\mathrm{f}}_{\mathrm{x}, \mathrm{x}}^{\mathrm{A}}\left(\omega_{\mathrm{k}}\right)$ é assintoticamente não viciado e consistente com distribuição assintoticamente normal. Maiores detalhes, ver ARA (1982). 


\subsection{Análise Discriminante- Definições}

Os objetivos de uma análise discriminante, segundo Johnson e Wichern (1982) são:

a) Descrever os padrões diferenciais de observações, ou séries, oriundas de várias populações conhecidas, através da determinação de funções denominadas discriminantes ou separadoras, cujos valores numéricos permitam distinguir, tanto quanto possivel, as referidas populações;

b) Atribuir novas observações, ou séries, a alguma dessas populações através de um critério que minimize o erro de classificação.

Definição 2.3.1 Seja $\Omega$ o espaço amostral associado ao vetor aleatório $X=\left[\begin{array}{llll}x_{1} & x_{2} & \cdots & x_{p}\end{array}\right]^{t}$. Definimos como partição de $\Omega$, os subconjuntos $R_{1}, R_{2}, \ldots, R_{g}$ tal que $\bigcup_{i=1}^{g} R_{i}=\Omega$ e $R_{i} \cap R_{j}=\phi$. para $i \neq j$ com $R i \neq \phi, i=1, \ldots, g$

Definição 2.3.2 Sejam $f_{1}(x), \ldots f_{g}(x)$, funções densidades associadas a um vetor de variáveis aleatórias, $\quad X=\left[\begin{array}{lllll}x_{1} & x_{2} & . & x_{p}\end{array}\right]^{t}$ das populações $\pi_{1}, \ldots, \pi_{9}$ respectivamente. Seja $R_{i} i=1 \ldots, g$ uma partição de $\Omega$, tal que $R_{i}$ seja o conjunto de valores de $\mathbf{X}$ para o qual classificamos um indivíduo como sendo proveniente da população $\pi_{i}$. Definimos 
a) Probabilidade total de má classificação (PTM):

$$
\operatorname{PTM}(\mathbf{X})=\sum_{i=1}^{g} \sum_{j=i} p(j \mid i) p_{i}
$$

b) Custo esperado de má classificação (CEM):

$$
\operatorname{CEM}(\mathbf{X})=\sum_{\mathbf{i}=1}^{\mathrm{g}} \sum_{\mathrm{j} \neq \mathrm{i}} \mathrm{c}(\mathrm{j} \mid \mathrm{i}) \mathrm{p}(\mathrm{j} \mid \mathrm{i}) \mathrm{p}_{\mathrm{i}}
$$

onde

$$
\begin{aligned}
& \mathrm{c}(j \mid \mathrm{i})=\text { custo da classificação na população } \pi_{\mathrm{j}} \text {, quando } X \text { pertence à } \\
& \text { população } \pi_{\mathrm{i}}, \\
& \mathrm{p}(\mathrm{j} \mid \mathrm{i})=\mathrm{P}\left\{\mathrm{X} \in \mathrm{R}_{\mathrm{j}} \mid \pi_{\mathrm{i}}\right\}=\int_{\mathrm{R}_{\mathrm{j}}} \mathrm{f}_{\mathrm{i}}(\mathbf{X}) \mathrm{dx}, \\
& \mathrm{p}_{\mathrm{i}}=\text { probabilidade a priori da população } \pi_{\mathrm{i}} .
\end{aligned}
$$

Um vetor $X$ será classificado como sendo proveniente da população $\pi_{k}$ se

$$
\min _{i} g_{i}(\mathbf{X})=\min _{i} \sum_{\substack{i=1 \\ i=k}}^{\underline{g}} p_{i} f_{i}(\mathbf{X}) c(k \mid i)
$$

ocorrer para $\mathrm{i}=\mathrm{k}$. Em caso de empate, $\mathrm{X}$ pode ser classificado indiferentemente entre as duas populações que apresentaram igual valor de $g_{i}(\mathbf{X})$.

$$
\text { Supondo o caso onde } c(i / j)=\left\{\begin{array}{lll}
1 & \text { se } & i=j \\
0 & \text { se } & i \neq j
\end{array}, \quad(2.47)\right. \text { reduz-se a }
$$




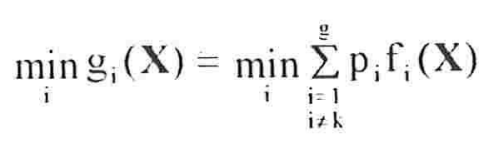

Se $X \sim N_{p}\left(\mu_{i} \Sigma_{i}\right), i=1, \ldots, g$, quando associado a população $\pi_{1}, \ldots, \pi_{g}$ respectivamente e os custos de má classificação forem iguais, um novo elemento será classificado como sendo da população $\pi_{\mathrm{k}}$ se

$$
g_{i}(\mathbf{X})=p_{i} f_{i}(X)=p_{i}(2 \pi)^{-p 2}\left|\Sigma_{i}\right|^{-1} \exp \left(-\frac{1}{2}\left(\mathbf{X}-\mu_{i}\right)^{\prime} \Sigma_{i}^{-1}\left(\mathbf{X}-\mu_{i}\right)\right)
$$

assumir o valor máximo para $\mathrm{i}=\mathrm{k}$.

Aplicando-se o logaritmo em (2.49), definimos o escore discriminante quadrático $\left(h_{i}(X)\right)$ como sendo:

$$
h_{i}(X)=-\frac{1}{2} \ln \left|\sum_{i}\right|-\frac{1}{2}\left(X-\mu_{i}\right)^{\prime} \sum_{i}^{-1}\left(X-\mu_{i}\right)^{\theta}+\ln p_{i}
$$

onde os parâmetros $\mu_{i}$ e $\sum_{i}, i=1, \ldots, g$ serão estimados a partir de uma amostra à qual denominamos de amostra de treinamento. Assim, um novo elemento será classificado como sendo da população $\pi_{k}$ se $h_{i}(\mathbf{X})$, dado por (2.50), assumir o valor máximo para $\mathrm{i}=\mathrm{k}$.

Definição 2.3.3 A taxa aparente de erro (TAE) é a fração das observações da amostra de treinamento que são classificadas de forma errada. Assim, utilizandose a matriz de confusão, o qual representa a real classificação versus a classificação prevista pela função, temos: 


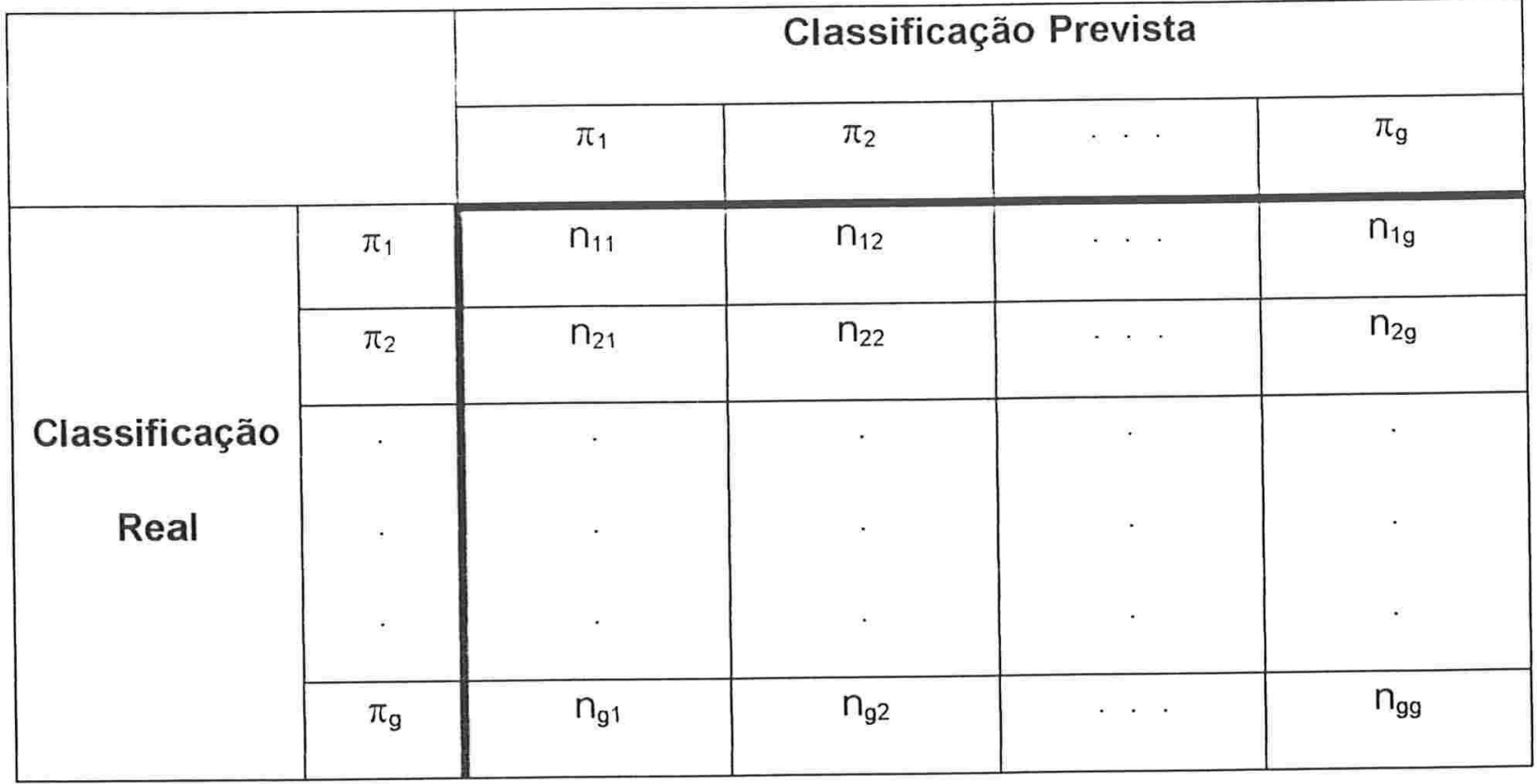

e

$$
T A E=\frac{\sum_{i=1}^{g} \sum_{j \neq i} n_{i j}}{n},
$$

onde $\mathrm{n}=\sum_{\mathrm{i}=1}^{\underline{g}} \sum_{\mathrm{i}=1}^{\underline{g}} \mathrm{n}_{\mathrm{ij}}$. Para mais detalhes, ver Johnson e Wichern (1982). 


\section{Capítulo 3}

\section{Análise Discriminante em Séries Temporais}

\subsection{Introdução}

Quando encontramos uma nova pessoa, a nossa tendência natural é tentar conhecer melhor esta pessoa (suas características mais marcantes, seu modo de agir,..) ou seja, reconhecer naquela pessoa alguns padrões que possam caracterizá-la e diferenciá-la das demais. Assim, parece natural a tentativa de reconhecimento de padrões em cada coisa que nos é apresentada, como forma de entendimento e seleção de objetos e pessoas que nos agradam ou não.

Desta forma, podemos caracterizar um problema de discriminação como sendo uma tentativa de classificação de uma nova observação em relação a grupos previamente existentes, dentre os quais temos interesse especial em alguns deles. Portanto, a análise discriminatória ou classificatória tem como objetivo básico alocar um novo indivíduo dentro de um grupo, a partir de um conjunto de variáveis que servirão para indicar os motivos das diferença entre os grupos. Além disso, "quantificar" o grau de semelhança entre os grupos e entre uma observação e cada grupo, constitui uma medida útil na realização de prognósticos e determinação no grau de certeza na tomada de decisão.

A aplicação da técnica de análise discriminante tradicional, no contexto de séries temporais (por exemplo em dados provenientes de um eletroencefalograma), fica comprometida devido ao número de parâmetros a serem estimados, principalmente em virtude do fato do número de indivíduos analisados, na maioria das vezes, ser muito menor do que o tamanho das séries, 
impossibilitando o cálculo da matriz de covariância sem a suposição de um modelo. Uma possivel solução para este problema, seria a construção de variáveis resumo, as quais. entretanto, além de acarretarem a perda de toda a informação da estrutura de correlação entre as observações, nem sempre são facilmente identificadas.

Uma alternativa para viabilizar a aplicação desta técnica no contexto de séries temporais, é a realização de uma redução no número de parâmetros a serem estimados, a partir da imposição de um modelo aos dados. Esta forma, apesar de sua simplicidade, apresenta alguns problemas que devem ser observados:

a) O primeiro, de ordem prática, tem seu foco voltado principalmente na necessidade da realização da operação de ajuste de modelos para um número grande de séries, sendo portanto necessário a utilização de métodos automáticos para ajuste dos mesmos;

b) Tendo definido um modelo para cada série, faz-se preemente a determinação de um modelo único que represente cada grupo. Neste caso, sugerimos a escolha de modelos de uma mesma familia (por exemplo, modelos auto-regressivos) juntamente com a utilização de média (ou mediana) dos parâmetros estimados pode representar uma possivel solução;

c) Tendo obtido os modelos de cada grupo, é necessário a determinação de uma função que minimize o CEM, que para o caso normal, possue uma expressão semelhante a do caso clássico. 
Outra alternativa para a realização da análise discriminante é a mudança de base para o domínio da frequência, de modo a possibilitar a utilização das propriedades assintóticas da transformada de Fourier. Entretanto, na estimação da variância da transformada (espectro), estimadores distintos devem ser testados visando determinar aqueles que possam apresentar melhores desempenhos. Dentre os estimadores espectrais serão utilizados neste trabalho o periodograma, o periodograma suavisado e o estimador espectral autoregressivo, definidos anteriormente. Outro problema que deve ser analisado é a limitação imposta por este tipo de análise que necessita que as séries estudadas apresentem mesmo tamanho. Neste caso, sugerimos neste trabalho, a utilização do estimador espectral auto-regressivo tomando-se, entretanto, frequências únicas para todas as séries. 


\subsection{Análise Discriminante no Domínio do Tempo}

Para o desenvolvimento da técnica de análise discriminante no domínio do tempo, utilizaremos os modelos $\operatorname{ARMA}(p, q)$ propostos por Box-Jenkins, ver Box, Jenkins e Reinsel (1994) visando a redução do número de parâmetros a serem estimados em cada série. Desta forma, podemos representar cada série na forma

$$
\phi^{(i, j)}(B)\left[X_{i j}(t)-\mu_{i}\right]=\theta^{(i, j)}(B) a_{i j}(t) \quad,
$$

$i=1, \ldots, g$ e $j=1, \quad, n_{i}$

onde

$\phi^{(\mathrm{i}, \mathrm{j})}(\mathrm{B})=1-\phi_{1}^{(\mathrm{i}, \mathrm{j})} \mathrm{B}-\phi_{2}^{(\mathrm{i}, \mathrm{j})} \mathrm{B}^{2}-\ldots-\phi_{\mathrm{p}}^{(\mathrm{i}, \mathrm{j})} \mathrm{B}^{\mathrm{p}}$

$\theta^{(\mathrm{i}, \mathrm{j})}(\mathrm{B})=1-\theta_{1}^{(\mathrm{i}, \mathrm{j})} \mathrm{B}-\theta_{2}^{(\mathrm{i}, \mathrm{j})} \mathrm{B}^{2}-\ldots-\theta_{\mathrm{q}}^{(\mathrm{i}, \mathrm{j})} \mathrm{B}^{\mathrm{q}}$

$X_{\mathrm{ij}}(\mathrm{t})$ : Representa a j-ésima série temporal do i-ésimo grupo,

$\mu_{\mathrm{i}} \quad$ : é a média da i-ésima população,

$\mathrm{a}_{\mathrm{ij}}(\mathrm{t})$ : ruído branco Gaussiano

O modelo (3.1) pode ser reescrito na forma invertida, ou seja,

$$
\pi^{(i . j)}(B)\left[X_{i j}(t)-\mu_{i}\right]=a_{i j}(t)
$$

$i=1, \ldots, g$ e $j=1, \quad, n_{i}$.

onde

$$
\pi^{(\mathrm{i} . \mathrm{j})}(\mathrm{B})=\frac{\phi^{(\mathrm{i} . \mathrm{j})}(\mathrm{B})}{\theta^{(\mathrm{i} . \mathrm{j})}(\mathrm{B})}=1-\pi_{1}^{(\mathrm{i} . \mathrm{j})} \mathrm{B}-\pi_{2}^{(\mathrm{i} . \mathrm{j})} \mathrm{B}^{2}-\ldots
$$


Tal parametrização tem por finalidade estabelecer uma classe de modelos de forma a possibilitar a constituição de um modelo único para cada grupo. A escolha de modelos deste tipo faz-se mais conveniente em virtude de sua maior facilidade de interpretação e do fato que, independente da ordem do modelo autoregressivo, sempre existe um valor $p$ tal que:

$$
\left[\mathrm{X}_{\mathrm{ij}}(\mathrm{t})-\mu_{\mathrm{i}}\right] \approx \sum_{\mathrm{k}=1}^{\mathrm{p}} \pi_{\mathrm{k}}^{(\mathrm{i} . \mathrm{j})}\left[\mathrm{X}_{\mathrm{ij}}(\mathrm{t}-\mathrm{k})-\mu_{\mathrm{i}}\right]+\mathrm{a}_{\mathrm{ij}}(\mathrm{t})
$$

onde $a_{i j}(t)$ é um ruído branco e estimadores dos parâmetros $\hat{\pi}_{k}^{(i . j)}, k=1, \ldots, p, e$ $\hat{\sigma}_{a_{i j}}$ podem ser obtidos a partir das equações de Yule-Walker, ver Priestley (1981).

Em seguida, visando a escolha de um modelo único para representar cada um dos grupos, procederemos da seguinte forma:

a) Dentro de cada grupo, calculamos $K_{i}=$ máximo das ordens dos modelos auto-regressivos associados a cada uma das séries pertencentes ao iésimo grupo, $\mathrm{i}=1, \ldots, \mathrm{g}$,

b) Reajustamos a cada série do i-ésimo grupo um modelo $A R\left(K_{i}\right)$, obtendose as estimativas $\hat{\pi}_{\mathrm{h}}^{(\mathrm{i} . \mathrm{j})}, \mathrm{k}=1, \ldots, \mathrm{K}_{\mathrm{i}} ; \mathrm{i}=1, \ldots, \mathrm{g} ; \mathrm{j}=1, \ldots, \mathrm{n}_{\mathrm{i}}$

c) Neste trabalho, sugerimos para a obtenção do modelo único de cada grupo os seguintes procedimentos, a saber:

i) A partir da média dos coeficientes do modelo auto-regressivo ponderado pelo tamanho da série: 


$$
\bar{\pi}_{k}^{(i)}=\frac{\sum_{j=1}^{n_{i}} n_{i j} \hat{\pi}_{k}^{(i . j)}}{\sum_{j=1}^{n_{i}} n_{i j}}
$$

onde $\mathrm{n}_{\mathrm{ij}}$ representa o tamanho da série do i-ésimo grupo,

$$
i=1, \ldots, g \text { e } k=1, \ldots, p .
$$

ii) A partir da mediana dos coeficientes de cada série pertencente a cada grupo:

$$
\tilde{\pi}_{\mathrm{k}}^{(\mathrm{i})}=\operatorname{mediana}\left(\hat{\pi}_{\mathrm{k}}^{(\mathrm{i}, 1)}, \ldots, \hat{\pi}_{\mathrm{k}}^{\left(\mathrm{i}, \mathrm{n}_{\mathrm{i}}\right)}\right)
$$

para $\mathrm{i}=1, \ldots, \mathrm{g}$ e $\mathrm{k}=1, \ldots, \mathrm{p}$.

Assim, podemos escrever o logaritmo da função de verossimilhança condicional de cada grupo,ver Box, Jenkins e Reinsel (1994), na forma:

$$
\ln \left\{L_{i}[X(p), \ldots, X(T-1) / X(0), \ldots, X(p-1)]\right\} \cong-\frac{(T-p)}{2} \ln \left(\sigma_{a_{i}}^{2}\right)-\frac{1}{2 \sigma_{a_{i}}^{2}} \sum_{t=p}^{T-1} a_{i}^{2}(t)
$$

Utilizando (2.50) e (3.7), temos que o escore discriminante quadrático, para o caso de uma série modelada por (3.1) será dado por

$$
\hat{h}_{i}(\mathbf{X})=-\frac{(T-p)}{2} \ln \left(\sigma_{a_{i}}^{2}\right)-\frac{1}{2 \sigma_{a_{i}}^{2}} \sum_{i=p+1}^{T-1} a_{i}^{2}(t)+\ln p_{i}
$$

e, assim, a série temporal $\{X(t), t=0,1, \ldots, T-1\}$ será classificada como proveniente da população $\pi_{k}$ se o escore discriminante quadrático, $h_{i}(X)$, assumir o valor máximo para $\mathrm{i}=\mathrm{k}$. 
Aplicando-se o filtro linear obtido a partir dos coeficientes estimados utilizando (3.5) ou (3.6), sobre cada nova série a ser classificada, calculamos $\hat{\sigma}_{\text {a }}$ e $\hat{\pi}_{k}^{(i . j)}$ que substituidos em (3.8) fornece:

$$
\hat{h}_{i}(X)=-\frac{(T-p)}{2} \ln \left(\hat{\sigma}_{a_{i}}^{2}\right)-\frac{1}{2 \hat{\sigma}_{a_{i}}^{2}} \sum_{i=p+1}^{T-1} \hat{a}_{i}^{2}(t)+\ln p_{i}
$$

que é denominado escore discriminante quadrático estimado que será utilizado para a alocação de uma nova série $X(t)$, em um dos grupos considerados para a análise. 


\subsection{Análise Discriminante no Domínio da Frequência}

Diferentemente do procedimento realizado no domínio do tempo, em que utilizamos a modelagem como forma de redução do número de parâmetros a serem estimados, no domínio da frequência, as propriedades assintóticas apresentadas no Teorema (2.1) e (2.2) possibilitam a utilização da análise discriminante tradicional em cada frequência.

Utilizando o Teorema 2.2, podemos escrever o logaritmo da função de verossimilhança de $d_{k}(x), k=0,1, \ldots, T-1$, sob a população $\pi_{i}$ como sendo

$$
\text { - } \ln \left\{\mathrm{L}\left(\mathrm{d}_{\mathrm{k}}(0), \ldots, \mathrm{d}_{\mathrm{k}}(\mathrm{T}-1)\right)\right\} \cong-\frac{1}{2} \sum_{\mathrm{k}=0}^{\mathrm{T}-1} \ln \mathrm{f}_{\mathrm{x}_{\mathrm{i}} \mathrm{x}_{\mathrm{i}}}\left(\omega_{\mathrm{k}}\right)-\frac{1}{2} \sum_{\mathrm{k}=0}^{\mathrm{T}-1} \frac{\left|\mathrm{d}_{\mathrm{x}_{\mathrm{i}}}(\mathrm{k})-\mathrm{d}_{\mu_{\mathrm{i}}}(\mathrm{k})\right|^{2}}{\mathrm{f}_{\mathrm{x}_{\mathrm{i}} \mathrm{x}_{\mathrm{i}}}\left(\omega_{\mathrm{k}}\right)}
$$

onde

- $\mathrm{f}_{\mathrm{x}_{\mathrm{i}} \mathrm{x}_{1}}\left(\omega_{\mathrm{k}}\right)$ é, como definido em (2.25), o espectro de $\mathrm{X}_{\mathrm{i}}(\mathrm{t})$, série pertencente à população $\pi_{\mathrm{i}}$, na frequência $\omega_{\mathrm{k}}=\frac{2 \pi \mathrm{k}}{\mathrm{T}}$, com a restrição $\mathrm{f}_{\mathrm{x}_{\mathrm{i}} \mathrm{x}_{\mathrm{i}}}\left(\omega_{\mathrm{k}}\right) \neq 0, \mathrm{e}$

- $\mathrm{d}_{\mu_{1}}(\mathrm{k})=(2 \pi \mathrm{T})^{1 / 2} \sum_{\mathrm{t}=0}^{\mathrm{T}-1} \mu_{\mathrm{i}} \exp \left(-\mathrm{i} \omega_{\mathrm{k}} \mathrm{t}\right)$ é a transformada de Fourier da média da série $X_{i}(t)$.

Assim, de forma análoga ao realizado no domínio do tempo, a partir de (2.50) e (3.10) construimos o escore discriminante quadrático. 


$$
h_{i}(\mathbf{X})=-\frac{1}{2} \sum_{k=0}^{T-1} \ln f_{x_{i} x_{i}}\left(\omega_{k}\right)-\frac{1}{2} \sum_{k=0}^{T-1} \frac{\left|d_{x_{i}}(k)-d_{\mu_{i}}(k)\right|^{2}}{f_{x_{i} x_{i}}\left(\omega_{k}\right)}+\ln p_{i} .
$$

A partir da amostra de treinamento, podemos obter, segundo Shumway(1988), os seguintes estimadores para $d_{\mu_{i}}(k)$ e $f_{x_{i} x_{i}}\left(\omega_{k}\right)$ :

$$
\begin{gathered}
\bar{d}_{x_{i}}(k)=n_{i}^{-1} \sum_{j=1}^{n_{i}} d_{x_{i j}}(k) \\
\hat{f}^{(1)}{ }_{x_{i} x_{i}}\left(\omega_{k}\right)=\left(n_{i}-1\right)^{-1} \sum_{j=1}^{n_{i}}\left|d_{x_{i}}(k)-\bar{d}_{x_{i}}(k)\right|^{2}
\end{gathered}
$$

onde

- $\mathrm{d}_{\mathrm{x}_{\mathrm{ij}}}(\mathrm{k})$ é a transformada de Fourier de j-ésima série do i-ésimo grupo $i=1, \ldots, g$ e $j=1, \ldots, n_{i}$

- $n_{i}$ é o número de séries do grupo i na amostra de treinamento.

De modo que, substituindo (3.12) e (3.13) em (3.11) temos:

$$
\hat{\mathrm{h}}_{\mathrm{i}}(\mathbf{X})=-\frac{1}{2} \sum_{\mathrm{k}=0}^{\mathrm{T}-1} \ln \hat{\mathrm{f}}^{(1)}{ }_{\mathrm{x}_{\mathrm{i}} \mathrm{x}_{\mathrm{i}}}\left(\omega_{\mathrm{k}}\right)-\frac{1}{2} \sum_{\mathrm{k}=0}^{\mathrm{T}-1} \frac{\left.\mid \mathrm{d}_{\mathrm{x}_{\mathrm{i}}}(\mathrm{k})-\overline{\mathrm{d}}_{\mathrm{x}_{\mathrm{i}}}(\mathrm{k})\right)\left.\right|^{2}}{\hat{\mathrm{f}}^{(\mathrm{i})}{ }_{\mathrm{x}_{\mathrm{i}} \mathrm{x}_{\mathrm{i}}}\left(\omega_{\mathrm{k}}\right)}+\ln \mathrm{p}_{\mathrm{i}}
$$

e classificamos uma nova série $X(t)$ como proveniente da população $\pi_{k}$ se $\hat{\mathrm{h}}_{\mathrm{k}}(\mathbf{X})>\hat{\mathrm{h}}_{\mathrm{j}}(\mathbf{X}), \quad \forall \mathrm{i} \neq \mathrm{k}$

Além do estimador para $\mathrm{f}_{\mathrm{x}_{\mathrm{i}} \mathrm{x}_{\mathrm{i}}}\left(\omega_{\mathrm{k}}\right)$ apresentado acima, Shumway (1988) propõe o estimador suavizado: 


$$
\hat{f}_{\mathrm{x}_{1} \mathrm{x}_{1}}^{(1)}\left(\mathrm{O}_{\mathrm{h}}\right)=\mathrm{L}^{-1} \sum_{1-\frac{(\mathrm{L}-\mathrm{-})}{2}}^{(\mathrm{L}-1)} \hat{f}_{\mathrm{x}_{1} \mathrm{~K}_{1}}^{(1)}\left(\omega_{\mathrm{h}}-\frac{2 \pi \mathrm{l}}{\mathrm{T}}\right)
$$

Neste trabalho, propomos também, a utilização do estimador espectral autoregressivo dado por:

$$
\overline{\mathrm{f}}_{\mathrm{x}_{1} \mathrm{x}_{\mathrm{i}}}^{A}\left(\omega_{k}\right)=\frac{\sum_{\mathrm{j}=1}^{\mathrm{n}_{1}} \hat{\mathrm{f}}_{\mathrm{x}_{i} \mathrm{x}_{\mathrm{i}}}^{\lambda}\left(\omega_{k}\right)}{\mathrm{n}_{\mathrm{i}}}
$$

onde $\overline{\mathrm{f}}_{\mathrm{x}_{\mathrm{i}} \mathrm{x}_{\mathrm{i}}}^{\mathrm{A}}\left(\omega_{\mathrm{k}}\right)$ é dado por (2.44) e $\mathrm{X}_{\mathrm{ij}}$ é a j-ésima série do i-ésimo grupo, $\mathrm{i}=1, \ldots \mathrm{g}$ e $j=1, \ldots, n_{i}$, 


\section{Capítulo 4}

\section{Simulação}

\subsection{Introdução}

Com o intuito de se verificar o desempenho de cada método, construimos 12 conjuntos com séries de tamanho 1000, pertencentes a dois grupos distintos. Utilizando-se 20 séries (10 de cada grupo), geramos amostras de aprendizagem dos seguintes modelos, todos $\operatorname{com} \mu=0$ e $\sigma_{a}^{2}=1$ :

A) Grupo 1: $A R(1) \operatorname{com} \phi=0,3$

Grupo 2: $A R(1) \operatorname{com} \phi=0,6$

B) Grupo 1: $A R(1) \operatorname{com} \phi=0,4$

Grupo 2: $A R(1) \operatorname{com} \phi=0,5$

C) Grupo 1: $A R(1) \operatorname{com} \phi=0,3$

Grupo 2: $\mathrm{AR}(1)$ com $\phi=0,9$

D) Grupo 1: $\mathrm{MA}(1) \operatorname{com} \theta=0,3$

Grupo 2: $\mathrm{MA}(1) \operatorname{com} \theta=0,6$

E) Grupo 1: $M A(1) \operatorname{com} \theta=0,4$

Grupo 2: $M A(1) \operatorname{com} \theta=0,5$

F) Grupo 1: $M A(1) \operatorname{com} \theta=0,3$

Grupo 2: $M A(1) \operatorname{com} \theta=0,9$

G) Grupo 1: $\operatorname{ARMA}(1,1) \operatorname{com} \phi=0.3$ e $\theta=0.1$

Grupo 2: $\operatorname{ARMA}(1,1) \operatorname{com} \phi=0.6$ e $\theta=0.2$

H) Grupo 1: $\operatorname{ARMA}(1,1) \operatorname{com} \phi=0.4$ e $\theta=0.1$

Grupo 2: $\operatorname{ARMA}(1,1) \operatorname{com} \phi=0.5$ e $\theta=0.2$ 
I) Grupo 1: $\operatorname{ARMA}(1,1) \operatorname{com} \phi=0.3$ e $\theta=0.9$

Grupo 2: $\operatorname{ARMA}(1,1) \operatorname{com} \phi=0.6$ e $\theta=0.9$

J) Grupo 1: $\operatorname{SARIMA}(0,1,1)(0,1,1)_{12} \operatorname{com} \theta=0,3$ e $\Theta=0.4$

Grupo 2: $\operatorname{SARIMA}(0,1,1)(0,1,1)_{12} \operatorname{com} \theta=0,3$ e $\Theta=0.7$

K) Grupo 1: $\operatorname{SARIMA}(0,1,1)(0,1,1)_{12} \operatorname{com} \theta=0,3$ e $\Theta=0.4$

Grupo 2: SARIMA $(0,1,1)(0,1,0)_{12} \operatorname{com} \theta=0,3$

L) Grupo 1: $\operatorname{SARIMA}(0,1,1)(0,1,1)_{12} \operatorname{com} \theta=0,3$ e $\Theta=0.4$

Grupo 2: $\operatorname{SARIMA}(0,1,1)(0,1,1)_{9} \operatorname{com} \theta=0,3$ e $\Theta=0.4$

Posteriormente, para verificarmos a capacidade de discriminação dos diferentes métodos sob cada conjunto, foram geradas cem novas amostras de tamanho 1000 para cada um dos grupo estudados, construído as matrizes de confusão e determinado a taxa de erro, este último obtido a partir do quociente entre o número de séries classificadas de forma incorreta pelo número total de séries geradas (200 amostras). A escolha dos modelos apresentados tem como justificativa:

- Nos grupos A e B, nosso intuito foi de verificar, em modelos autoregressivos, qual a velocidade de perda do poder discriminante a medida em que há a aproximação dos valores dos parâmetros;

- No modelo C, tentamos identificar se a existência de parâmetros cujos valores estivessem próximos dos limites de estacionariedade comprometia o resultado de algum dos métodos; 
- Nos grupos D a F, de modo análogo aos grupos $A$ a $C$, estudamos o comportamento destes métodos em modelos de médias móveis (ressalvado que neste caso, os limites adotados foram os de invertibilidade);

- Nos grupos G a I, testamos os métodos em modelos $\operatorname{ARMA}(1,1)$, sendo entretanto estudados apenas os limites da invertibilidade;

- O grupo J, teve como intuito verificar se os métodos, em modelos sazonais com mesma periodicidade conseguiam discriminar diferenças apenas na parte estocástica;

-No grupo K, elaboramos 2 grupos cuja único diferencial foi a existência ou não de uma periodicidade estocástica

-No grupo L, foram simulados 2 modelos cujo diferencial deveu-se à periodicidade de 12 em um dos grupos, e de 9 no outro. Este grupo teve como intuito verificar se, em modelos mais simples (uma única periodicidade), os métodos no domínio da freqüência teriam melhor desempenho do que os no domínio do tempo.

\subsection{Análise dos Resultados}

Após a simulação e construção das matrizes de confusão, apresentadas na Figura 4.1, foram determinadas as taxas de erro, para cada método, que são apresentadas na Tabela 4.2 e na Figura 4.2. Os modelos ajustados nos métodos no domínio do tempo são apresentados na Tabela 4.1. 
Tabela 4.1 - Ordens dos modelos ajustados, média e mediana dos coeficientes

\begin{tabular}{|c|c|c|c|c|c|c|c|c|}
\hline \multirow{3}{*}{$\begin{array}{l}\text { Coefici- } \\
\text { entes }\end{array}$} & \multicolumn{4}{|c|}{ Conjunto $A$} & \multicolumn{4}{|c|}{ Conjunto B } \\
\hline & \multicolumn{2}{|c|}{ Grupo 1} & \multicolumn{2}{|c|}{ Grupo 2} & \multicolumn{2}{|c|}{ Grupo 1} & \multicolumn{2}{|c|}{ Grupo 2} \\
\hline & Média & Mediana & Média & Mediana & Média & Mediana & \begin{tabular}{|l|l|l} 
Média & I \\
\end{tabular} & Mediana \\
\hline $\begin{array}{l}\text { Ordem } \\
\text { minima } \\
\text { obtida }\end{array}$ & \multicolumn{2}{|c|}{\begin{tabular}{|c|}
1 \\
\end{tabular}} & \multicolumn{2}{|r|}{1} & \multicolumn{2}{|r|}{1} & \multicolumn{2}{|c|}{1} \\
\hline $\begin{array}{l}\text { Ordem } \\
\text { máxima } \\
\text { obtida }\end{array}$ & \multicolumn{2}{|c|}{16} & \multicolumn{2}{|r|}{6} & \multicolumn{2}{|c|}{8} & \multicolumn{2}{|c|}{17} \\
\hline$\phi_{1}$ & 0,3045 & 0,2928 & 0,6011 & 0,6071 & 0,3670 & 0,3799 & 0,5018 & 0,5008 \\
\hline$\phi_{2}$ & $-0,0173$ & 0,0000 & 0,0037 & 0,0057 & $-0,0163$ & 0,0167 & 0,0170 & 0,0031 \\
\hline$\phi_{3}$ & 0,0239 & 0,0122 & 0,0027 & $-0,0012$ & 0,0337 & 0,0072 & $-0,0018$ & 0,0019 \\
\hline$\phi_{4}$ & $-0,0327$ & 0,0006 & $-0,0055$ & $-0,0077$ & $-0,0188$ & $-0,0184$ & $-0,0166$ & $-0,0100$ \\
\hline$\phi_{5}$ & $-0,0064$ & 0,0173 & 0,0063 & 0,0101 & 0,0133 & 0,0144 & 0,0044 & $-0,0061$ \\
\hline$\phi_{6}$ & $-0,0058$ & 0,0010 & $-0,0099$ & $-0,0090$ & $-0,0280$ & $-0,0193$ & $-0,0013$ & 0,0093 \\
\hline$\phi_{7}$ & 0,0035 & 0,0100 & & & 0,0709 & $-0,0009$ & $-0,0034$ & 0,0040 \\
\hline$\phi_{8}$ & $-0,0133$ & 0,0130 & & & 0,0172 & 0,0012 & 0,0037 & 0,0052 \\
\hline$\phi_{9}$ & 0,0152 & 0,0211 & & & & & 0,0067 & 0,0060 \\
\hline$\phi_{10}$ & 0,0461 & 0,0021 & & & & & $-0,0066$ & $-0,0145$ \\
\hline$\phi_{11}$ & $-0,0498$ & 0,0159 & & & & & $-0,0062$ & $-0,0118$ \\
\hline$\phi_{12}$ & 0,0052 & 0,0060 & & & & & 0,0132 & 0,0065 \\
\hline$\phi_{13}$ & $-0,0167$ & 0,0004 & & & & & $-0,0078$ & $-0,0044$ \\
\hline$\phi_{14}$ & 0,0070 & 0,0105 & & & & & $-0,0109$ & $-0,0087$ \\
\hline$\phi_{15}$ & $-0,0139$ & 0,0110 & & & & & $-0,0023$ & $-0,0063$ \\
\hline$\phi_{16}$ & $-0,0125$ & 0,0041 & & & & & 0,0204 & 0,0203 \\
\hline$\phi_{17}$ & & & & & & & $-0,0040$ & 0,0057 \\
\hline
\end{tabular}


Tabela 4.1 - (Continuação)

\begin{tabular}{|c|c|c|c|c|c|c|c|c|}
\hline \multirow{3}{*}{$\begin{array}{l}\text { Coefici- } \\
\text { entes }\end{array}$} & \multicolumn{4}{|c|}{ Conjunto $\mathrm{C}$} & \multicolumn{4}{|c|}{ Conjunto D } \\
\hline & \multicolumn{2}{|c|}{ Grupo 1} & \multicolumn{2}{|c|}{ Grupo 2} & \multicolumn{2}{|c|}{ Grupo 1} & \multicolumn{2}{|c|}{ Grupo 2} \\
\hline & Média & Mediana & Média & Mediana & Média & Mediana & Média $\mid 1$ & Mediana \\
\hline $\begin{array}{l}\text { Ordem } \\
\text { minima } \\
\text { obtida }\end{array}$ & \multicolumn{2}{|r|}{1} & \multicolumn{2}{|c|}{1} & \multicolumn{2}{|c|}{1} & \multicolumn{2}{|c|}{3} \\
\hline $\begin{array}{l}\text { Ordem } \\
\text { máxima } \\
\text { obtida } \\
\end{array}$ & \multicolumn{2}{|c|}{4} & \multicolumn{2}{|r|}{5} & \multicolumn{2}{|c|}{11} & \multicolumn{2}{|c|}{11} \\
\hline$\phi_{1}$ & 0,3037 & 0,3048 & 0,8903 & 0,8830 & $-0,2440$ & $-0,2989$ & 0,5922 & $-0,5965$ \\
\hline$\phi_{2}$ & $-0,0504$ & 0,0096 & 0,0168 & $-0,0097$ & $-0,1139$ & $-0,0993$ & 0,3705 & $-0,3623$ \\
\hline$\phi_{3}$ & 0,0510 & $-0,0160$ & 0,0001 & 0,0032 & $-0,0251$ & $-0,0113$ & 0,2199 & $-0,2290$ \\
\hline$\phi_{4}$ & 0,0025 & $-0,0168$ & 0,0114 & 0,0192 & $-0,0011$ & $-0,0111$ & 0,1419 & $-0,1500$ \\
\hline$\phi_{5}$ & & & 0,0023 & $-0,0027$ & 0,0165 & 0,0198 & 0,0918 & $-0,0920$ \\
\hline$\phi_{6}$ & & & & & $-0,0337$ & 0,0110 & 0,0606 & $-0,0679$ \\
\hline$\phi_{7}$ & & & & & 0,0164 & 0,0259 & 0,0530 & $-0,0581$ \\
\hline$\phi_{8}$ & & & & & $-0,0101$ & $-0,0046$ & 0,0344 & $-0,0348$ \\
\hline$\phi_{9}$ & & & & & 0,0178 & 0,0028 & 0,0352 & $-0,0336$ \\
\hline$\cdot \phi_{10}$ & & & & & 0,0381 & 0,0068 & 0,0197 & $-0,0177$ \\
\hline$\phi_{11}$ & & & & & 0,0237 & 0,0030 & 0,0009 & 0,0064 \\
\hline
\end{tabular}


Tabela 4.1 - (Continuação)

\begin{tabular}{|c|c|c|c|c|c|c|c|c|}
\hline \multirow{3}{*}{$\begin{array}{l}\text { Coefici- } \\
\text { entes }\end{array}$} & \multicolumn{4}{|c|}{ Conjunto E } & \multicolumn{4}{|c|}{ Conjunto F } \\
\hline & \multicolumn{2}{|c|}{ Grupo 1} & \multicolumn{2}{|c|}{ Grupo 2} & \multicolumn{2}{|c|}{ Grupo 1} & \multicolumn{2}{|c|}{ Grupo 2} \\
\hline & Média & Mediana & \begin{tabular}{l|l} 
Média & I \\
\end{tabular} & Mediana & Média & Mediana & Média $\mathrm{N}$ & Mediana \\
\hline $\begin{array}{l}\text { Ordem } \\
\text { minima } \\
\text { obtida }\end{array}$ & \multicolumn{2}{|c|}{2} & \multicolumn{2}{|c|}{2} & \multicolumn{2}{|c|}{1} & \multicolumn{2}{|c|}{11} \\
\hline $\begin{array}{l}\text { Ordem } \\
\text { máxima } \\
\text { obtida } \\
\end{array}$ & \multicolumn{2}{|c|}{4} & \multicolumn{2}{|c|}{23} & \multicolumn{2}{|c|}{5} & \multicolumn{2}{|l|}{27} \\
\hline$\phi_{1}$ & $-0,3887$ & $-0,4017$ & 0,5195 & $-0,5184$ & $-0,3557$ & $-0,3101$ & $-0,9061$ & $-0,8988$ \\
\hline$\phi_{2}$ & $-0,1771$ & $-0,1532$ & 0,2568 & $-0,2557$ & $-0,0971$ & $-0,0872$ & $-0,8201$ & $-0,8155$ \\
\hline$\phi_{3}$ & $-0,0393$ & $-0,0599$ & 0,1297 & $-0,1157$ & $-0,0146$ & $-0,0300$ & $-0,7375$ & $-0,7467$ \\
\hline$\phi_{4}$ & $-0,0082$ & $-0,0183$ & 0,0617 & $-0,0454$ & $-0,0189$ & $-0,0288$ & $-0,6589$ & $-0,6479$ \\
\hline$\phi_{5}$ & & & 0,0459 & $-0,0504$ & $-0,0174$ & $-0,0169$ & $-0,5697$ & $-0,5630$ \\
\hline$\phi_{6}$ & & & 0,0201 & $-0,0203$ & & & $-0,5150$ & $-0,5029$ \\
\hline$\phi_{7}$ & & & 0,0081 & $-0,0164$ & & & $-0,4467$ & $-0,4561$ \\
\hline$\phi_{8}$ & & & 0,0105 & $-0,0015$ & & & $-0,4072$ & $-0,4027$ \\
\hline$\phi_{9}$ & & & 0,0143 & $-0,0149$ & & & $-0,3593$ & $-0,3398$ \\
\hline$\phi_{10}$ & & & 0,0006 & $-0,0128$ & & & $-0,3345$ & $-0,3342$ \\
\hline$\phi_{11}$ & & & 0,0128 & $-0,0187$ & & & $-0,3060$ & $-0,3196$ \\
\hline$\phi_{12}$ & & & 0,0084 & $-0,0101$ & & & $-0,2555$ & $-0,2627$ \\
\hline$\phi_{13}$ & & & 0,0002 & $-0,0024$ & & & $-0,2260$ & $-0,2425$ \\
\hline$\phi_{14}$ & & & 0,0101 & $-0,0110$ & & & $-0,2041$ & $-0,2023$ \\
\hline$\phi_{15}$ & & & 0,0221 & $-0,0221$ & & & $-0,1748$ & $-0,1645$ \\
\hline$\phi_{16}$ & & & 0,0148 & $-0,0139$ & & & $-0,1516$ & $-0,1403$ \\
\hline$\phi_{17}$ & & & 0,0064 & 0,0074 & & & $-0,1395$ & $-0,1219$ \\
\hline$\phi_{18}$ & & & 0,0096 & 0,0153 & & & $-0,1258$ & $-0,1067$ \\
\hline$\phi_{19}$ & & & 0,0037 & 0,0099 & & & $-0,1121$ & $-0,0986$ \\
\hline$\phi_{20}$ & & & 0,0191 & $-0,0178$ & & & $-0,0959$ & $-0,0844$ \\
\hline$\phi_{21}$ & & & 0,0038 & $-0,0084$ & & & $\begin{array}{l}-0,0702 \\
\end{array}$ & $-0,0650$ \\
\hline$\phi_{22}$ & & & 0,0183 & 0,0105 & & & $-0,0621$ & $-0,0706$ \\
\hline$\phi_{23}$ & & & 0,0149 & 0,0125 & & & $-0,0396$ & $-0,0455$ \\
\hline$\phi_{24}$ & & & & & & & $-0,0234$ & $-0,0332$ \\
\hline$\phi_{25}$ & & & & & & & $-0,0051$ & $-0,0056$ \\
\hline$\phi_{26}$ & & & & & & & 0,0041 & $-0,0070$ \\
\hline$\phi_{27}$ & & & & & & & 0,0042 & 0,0104 \\
\hline
\end{tabular}


Tabela 4.1 - (Continuação)

\begin{tabular}{|c|c|c|c|c|c|c|c|c|}
\hline \multirow{3}{*}{$\begin{array}{l}\text { Coefici- } \\
\text { entes }\end{array}$} & \multicolumn{4}{|c|}{ Conjunto G } & \multicolumn{4}{|c|}{ Conjunto $\mathrm{H}$} \\
\hline & \multicolumn{2}{|c|}{ Grupo 1} & \multicolumn{2}{|c|}{ Grupo 2} & \multicolumn{2}{|c|}{ Grupo 1} & \multicolumn{2}{|c|}{ Grupo 2} \\
\hline & Média & Mediana & Média & Mediana & Média & Mediana & Média $\mid \bar{I}$ & Mediana \\
\hline $\begin{array}{l}\text { Ordem } \\
\text { mínima } \\
\text { obtida }\end{array}$ & \multicolumn{2}{|c|}{1} & \multicolumn{2}{|c|}{2} & \multicolumn{2}{|c|}{1} & \multicolumn{2}{|c|}{1} \\
\hline $\begin{array}{l}\text { Ordem } \\
\text { máxima } \\
\text { obtida } \\
\end{array}$ & \multicolumn{2}{|c|}{4} & \multicolumn{2}{|c|}{5} & \multicolumn{2}{|c|}{2} & \multicolumn{2}{|c|}{16} \\
\hline$\phi_{1}$ & 0,1336 & 0,2029 & 0,3835 & 0,3836 & 0,3383 & 0,2995 & 0,2917 & 0,2984 \\
\hline$\phi_{2}$ & $-0,0577$ & 0,0130 & 0,0921 & 0,0922 & 0,0507 & 0,0212 & 0,0608 & 0,0577 \\
\hline$\phi_{3}$ & 0,0320 & 0,0084 & 0,0010 & $-0,0067$ & & & 0,0004 & 0,0083 \\
\hline$\phi_{4}$ & $-0,0122$ & $-0,0027$ & 0,0074 & 0,0151 & & & 0,0042 & 0,0001 \\
\hline$\phi_{5}$ & & & 0,0063 & $-0,0075$ & & & 0,0236 & 0,0265 \\
\hline$\phi_{6}$ & & & & & & & 0,0096 & 0,0048 \\
\hline$\phi_{7}$ & & & & & & & 0,0004 & 0,0075 \\
\hline$\phi_{8}$ & & & & & & & 0,0071 & 0,0092 \\
\hline$\phi_{9}$ & & & & & & & 0,0123 & 0,0168 \\
\hline$\phi_{10}$ & & & & & & & 0,0022 & 0,0095 \\
\hline$\phi_{11}$ & & & & & & & 0,0206 & 0,0381 \\
\hline$\phi_{12}$ & & & & & & & 0,0033 & 0,0083 \\
\hline$\phi_{13}$ & & & & & & & 0,0066 & 0,0067 \\
\hline$\phi_{14}$ & & & & & & & 0,0096 & 0,0227 \\
\hline$\phi_{15}$ & & & & & & & 0,0011 & 0,0023 \\
\hline$\phi_{16}$ & & & & & & & 0,0099 & 0,0105 \\
\hline
\end{tabular}


Tabela 4.1 - (Continuação)

\begin{tabular}{|c|c|c|c|c|c|c|c|c|}
\hline \multirow{3}{*}{$\begin{array}{l}\text { Coefici- } \\
\text { entes }\end{array}$} & \multicolumn{4}{|c|}{ Conjunto I } & \multicolumn{4}{|c|}{ Conjunto $\mathrm{J}$} \\
\hline & \multicolumn{2}{|c|}{ Grupo 1} & \multicolumn{2}{|c|}{ Grupo 2} & \multicolumn{2}{|c|}{ Grupo 1} & \multicolumn{2}{|c|}{ Grupo 2} \\
\hline & Média & Mediana & Média & Mediana & Média II & Mediana & Média $\mid \mathrm{N}$ & Mediana \\
\hline $\begin{array}{l}\text { Ordem } \\
\text { mínima } \\
\text { obtida }\end{array}$ & \multicolumn{2}{|c|}{1} & \multicolumn{2}{|c|}{1} & \multicolumn{2}{|c|}{1} & \multicolumn{2}{|c|}{1} \\
\hline $\begin{array}{l}\text { Ordem } \\
\text { máxima } \\
\text { obtida } \\
\end{array}$ & \multicolumn{2}{|c|}{7} & \multicolumn{2}{|c|}{11} & \multicolumn{2}{|c|}{30} & \multicolumn{2}{|c|}{26} \\
\hline$\phi_{1}$ & 0,2583 & 0,2145 & 0,4090 & 0,4263 & 0,9612 & 0,9931 & 0,9913 & 0,9989 \\
\hline$\phi_{2}$ & 0,0060 & 0,0031 & 0,0633 & 0,0683 & 0,0447 & 0,0005 & $-0,0330$ & 0,0191 \\
\hline$\phi_{3}$ & $-0,0153$ & 0,0052 & 0,0003 & $-0,0020$ & $-0,0502$ & 0,0005 & 0,0300 & 0,0090 \\
\hline$\phi_{4}$ & 0,0095 & $-0,0144$ & 0,0070 & 0,0012 & 0,0143 & 0,0030 & $-0,0076$ & 0,0016 \\
\hline$\phi_{5}$ & $-0,0459$ & $-0,0116$ & 0,0025 & 0,0036 & 0,0162 & 0,0073 & 0,0357 & 0,0257 \\
\hline$\phi_{6}$ & 0,0415 & 0,0234 & 0,0084 & 0,0161 & $-0,0432$ & 0,0191 & $-0,0138$ & 0,0122 \\
\hline$\phi_{7}$ & $-0,0233$ & 0,0095 & 0,0134 & 0,0101 & 0,0634 & 0,0120 & 0,0166 & 0,0056 \\
\hline$\phi_{8}$ & & & 0,0062 & $-0,0135$ & $-0,0496$ & 0,0049 & $-0,0363$ & 0,0231 \\
\hline$\phi_{9}$ & & & 0,0115 & $-0,0108$ & 0,0309 & 0,0054 & 0,0055 & 0,0005 \\
\hline$\phi_{10}$ & & & 0,0132 & $-0,0159$ & 0,0284 & 0,0017 & $-0,0199$ & 0,0127 \\
\hline$\phi_{11}$ & & & 0,0017 & $-0,0006$ & $-0,0226$ & 0,0045 & 0,0174 & 0,0121 \\
\hline$\phi_{12}$ & & & & & 0,1634 & 0,0687 & 0,1091 & 0,0689 \\
\hline$\phi_{13}$ & & & & & $-0,1891$ & 0,0707 & $-0,1051$ & 0,0596 \\
\hline$\phi_{14}$ & & & & & 0,0396 & 0,0023 & $-0,0253$ & 0,0237 \\
\hline$\phi_{15}$ & & & & & $-0,0365$ & 0,0022 & 0,0226 & 0,0086 \\
\hline$\phi_{16}$ & & & & & $-0,0055$ & 0,0050 & $-0,0073$ & 0,0040 \\
\hline$\phi_{17}$ & & & & & 0,0287 & 0,0059 & 0,0363 & 0,0262 \\
\hline$\phi_{18}$ & & & & & $-0,0497$ & 0,0158 & $-0,0135$ & 0,0118 \\
\hline$\phi_{19}$ & & & & & 0,0569 & 0,0175 & 0,0165 & 0,0057 \\
\hline$\phi_{20}$ & & & & & $-0,0414$ & 0,0176 & $-0,0348$ & 0,0239 \\
\hline$\phi_{21}$ & & & & & 0,0150 & 0,0133 & 0,0051 & 0,0016 \\
\hline$\phi_{22}$ & & & & & 0,0407 & 0,0057 & $-0,0236$ & 0,0129 \\
\hline$\phi_{23}$ & & & & & $-0,0280$ & 0,0015 & 0,0222 & 0,0161 \\
\hline$\phi_{24}$ & & & & & 0,1705 & 0,0688 & 0,1055 & 0,0652 \\
\hline$\phi_{25}$ & & & & & $-0,1802$ & 0,0713 & $-0,1049$ & 0,0596 \\
\hline$\phi_{26}$ & & & & & 0,0280 & 0,0051 & 0,0081 & 0,0040 \\
\hline$\phi_{27}$ & & & & & $-0,0231$ & 0,0004 & & \\
\hline$\phi_{28}$ & & & & & $-0,0196$ & 0,0061 & & \\
\hline$\phi_{29}$ & & & & & 0,0348 & 0,0000 & & \\
\hline$\phi_{30}$ & & & & & $-0,0031$ & 0,0043 & & \\
\hline
\end{tabular}


Tabela 4.1 - (Continuação)

\begin{tabular}{|c|c|c|c|c|c|c|c|c|}
\hline \multirow{3}{*}{$\begin{array}{l}\text { Coefici- } \\
\text { entes }\end{array}$} & \multicolumn{4}{|c|}{ Conjunto $\mathrm{K}$} & \multicolumn{4}{|c|}{ Conjunto L } \\
\hline & \multicolumn{2}{|c|}{ Grupo 1} & \multicolumn{2}{|c|}{ Grupo 2} & \multicolumn{2}{|c|}{ Grupo 1} & \multicolumn{2}{|c|}{ Grupo 2} \\
\hline & Média & Mediana & \begin{tabular}{l|l} 
Média \\
\end{tabular} & Mediana & Média & Mediana & Média $\mid \bar{I}$ & Mediana \\
\hline $\begin{array}{l}\text { Ordem } \\
\text { mínima } \\
\text { obtida }\end{array}$ & \multicolumn{2}{|c|}{1} & \multicolumn{2}{|r|}{1} & \multicolumn{2}{|c|}{1} & \multicolumn{2}{|c|}{1} \\
\hline $\begin{array}{l}\text { Ordem } \\
\text { máxima } \\
\text { obtida }\end{array}$ & \multicolumn{2}{|c|}{25} & \multicolumn{2}{|c|}{16} & \multicolumn{2}{|c|}{26} & \multicolumn{2}{|c|}{1} \\
\hline$\phi_{1}$ & 0,9800 & 0,9856 & \begin{tabular}{|l|}
0,9667 \\
\end{tabular} & 0,9690 & 0,9264 & 0,9518 & 0,9969 & 0,9969 \\
\hline$\phi_{2}$ & 0,0056 & 0,0016 & 0,0574 & 0,0531 & 0,0419 & 0,0183 & & \\
\hline$\phi_{3}$ & $-0,0199$ & 0,0004 . & $-0,0625$ & $-0,0579$ & 0,0255 & 0,0284 & & \\
\hline$\phi_{4}$ & 0,0226 & 0,0148 & 0,0651 & 0,0645 & 0,0465 & 0,0196 & & \\
\hline$\phi_{5}$ & 0,0043 & 0,0093 & $-0,0551$ & $-0,0601$ & 0,1132 & 0,0431 & & \\
\hline$\phi_{6}$ & 0,0378 & 0,0223 & 0,0604 & 0,0608 & 0,0716 & 0,0277 & & \\
\hline$\phi_{7}$ & $-0,0320$ & 0,0241 & $-0,0600$ & $-0,0603$ & 0,0189 & 0,0315 & & \\
\hline$\phi_{8}$ & 0,0200 & 0,0124 & 0,0563 & 0,0537 & 0,0429 & 0,0278 & & \\
\hline$\phi_{9}$ & $-0,0610$ & 0,0147 & $|-0,0674|$ & $-0,0613$ & 0,0143 & 0,0006 & & \\
\hline$\phi_{10}$ & 0,0215 & 0,0007 & 0,0635 & 0,0564 & 0,0397 & 0,0383 & & \\
\hline$\phi_{11}$ & $-0,0205$ & 0,0045 & $-0,0591$ & $-0,0585$ & 0,0417 & 0,0169 & & \\
\hline$\phi_{12}$ & 0,1653 & 0,0729 & 0,0822 & 0,0752 & 0,3865 & 0,2801 & & \\
\hline$\phi_{13}$ & $-0,1469$ & 0,0722 & $-0,0800$ & $-0,0703$ & 0,3587 & 0,2504 & & \\
\hline$\phi_{14}$ & 0,0058 & 0,0022 & 0,0559 & 0,0524 & 0,0322 & 0,0090 & & \\
\hline$\phi_{15}$ & $-0,0181$ & 0,0001 & $-0,0609$ & $-0,0569$ & 0,0291 & 0,0325 & & \\
\hline$\phi_{16}$ & 0,0275 & 0,0148 & $\mid 0,0344$ & 0,0320 & 0,0004 & 0,0068 & & \\
\hline$\phi_{17}$ & 0,0024 & 0,0083 & & & 0,0133 & 0,0253 & & \\
\hline$\phi_{18}$ & 0,0374 & 0,0245 & & & 0,0159 & 0,0283 & & \\
\hline$\phi_{19}$ & $-0,0362$ & 0,0232 & & & 0,0306 & 0,0138 & & \\
\hline$\phi_{20}$ & 0,0171 & 0,0114 & & & 0,0329 & 0,0351 & & \\
\hline$\phi_{21}$ & $-0,0555$ & 0,0152 & & & 0,0111 & 0,0052 & & \\
\hline$\phi_{22}$ & 0,0246 & 0,0004 & & & 0,0449 & 0,0327 & & \\
\hline$\phi_{23}$ & $-0,0179$ & 0,0045 & & & 0,0244 & 0,0231 & & \\
\hline$\phi_{24}$ & 0,1588 & 0,0739 & & & 0,3544 & 0,2639 & & \\
\hline$\phi_{25}$ & $-0,1269$ & 0,0665 & & & 0,3334 & 0,2535 & & \\
\hline$\phi_{26}$ & & & & & 0,0533 & 0,0355 & & \\
\hline
\end{tabular}


Tabela 4.2 - Taxa de erro dos diferentes métodos

\begin{tabular}{|c|c|c|c|c|c|c|c|}
\hline \multirow[t]{2}{*}{ Modelo } & \multicolumn{2}{|c|}{ Dominio do Tempo } & \multirow[t]{2}{*}{ Periodograma } & \multicolumn{3}{|c|}{ Periodograma Suavizado } & \multirow{2}{*}{$\begin{array}{l}\text { Estimador } \\
\text { espectral } \\
\text { Auto- } \\
\text { regressivo }\end{array}$} \\
\hline & Mediana & Média & & $L=10$ & $L=50$ & $L=100$ & \\
\hline$a$ & $0 \%$ & $0 \%$ & $1 \%$ & $0 \%$ & $0 \%$ & $0 \%$ & $0 \%$ \\
\hline $\mathrm{b}$ & $10,5 \%$ & $21,5 \%$ & $38,5 \%$ & $34 \%$ & $37 \%$ & $31,5 \%$ & $32,5 \%$ \\
\hline c & $0 \%$ & $0 \%$ & $0 \%$ & $0 \%$ & $0 \%$ & $0 \%$ & $0 \%$ \\
\hline d & $0 \%$ & $0 \%$ & $1 \%$ & $0 \%$ & $0 \%$ & $0 \%$ & $0 \%$ \\
\hline e & $4,5 \%$ & $9 \%$ & $40,5 \%$ & $37 \%$ & $33,5 \%$ & $40 \%$ & $38 \%$ \\
\hline$f$ & $0 \%$ & $0 \%$ & $0 \%$ & $0 \%$ & $0 \%$ & $0 \%$ & $0 \%$ \\
\hline $\mathrm{g}$ & $1 \%$ & $0 \%$ & $12,5 \%$ & $6,5 \%$ & $4,5 \%$ & $4,5 \%$ & $3,5 \%$ \\
\hline $\mathrm{h}$ & $49 \%$ & $50 \%$ & $57 \%$ & $53 \%$ & $51 \%$ & $50,5 \%$ & $50,5 \%$ \\
\hline $\mathrm{i}$ & $0 \%$ & $0 \%$ & $7,5 \%$ & $5 \%$ & $5 \%$ & $5 \%$ & $5,5 \%$ \\
\hline j & $50 \%$ & $50 \%$ & $44,5 \%$ & $47 \%$ & $45 \%$ & $40,5 \%$ & $42,5 \%$ \\
\hline k & $50 \%$ & $50 \%$ & $19 \%$ & $18,5 \%$ & $18 \%$ & $19,5 \%$ & $41 \%$ \\
\hline i & $50 \%$ & $50 \%$ & $58,5 \%$ & $58,5 \%$ & $58,5 \%$ & $58,5 \%$ & $45,5 \%$ \\
\hline
\end{tabular}




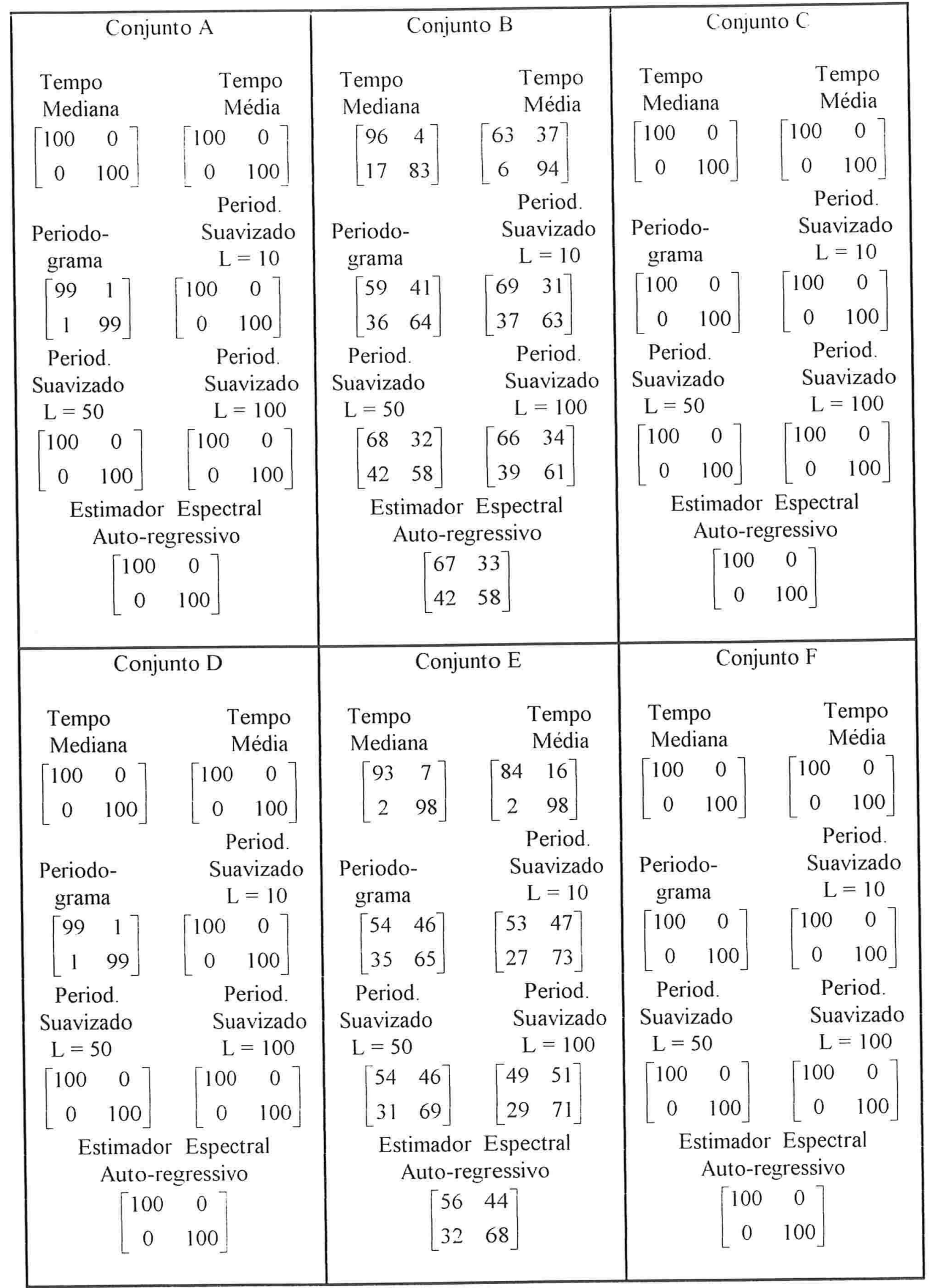

Figura 4.1: Matrizes de confusão dos diferentes métodos 


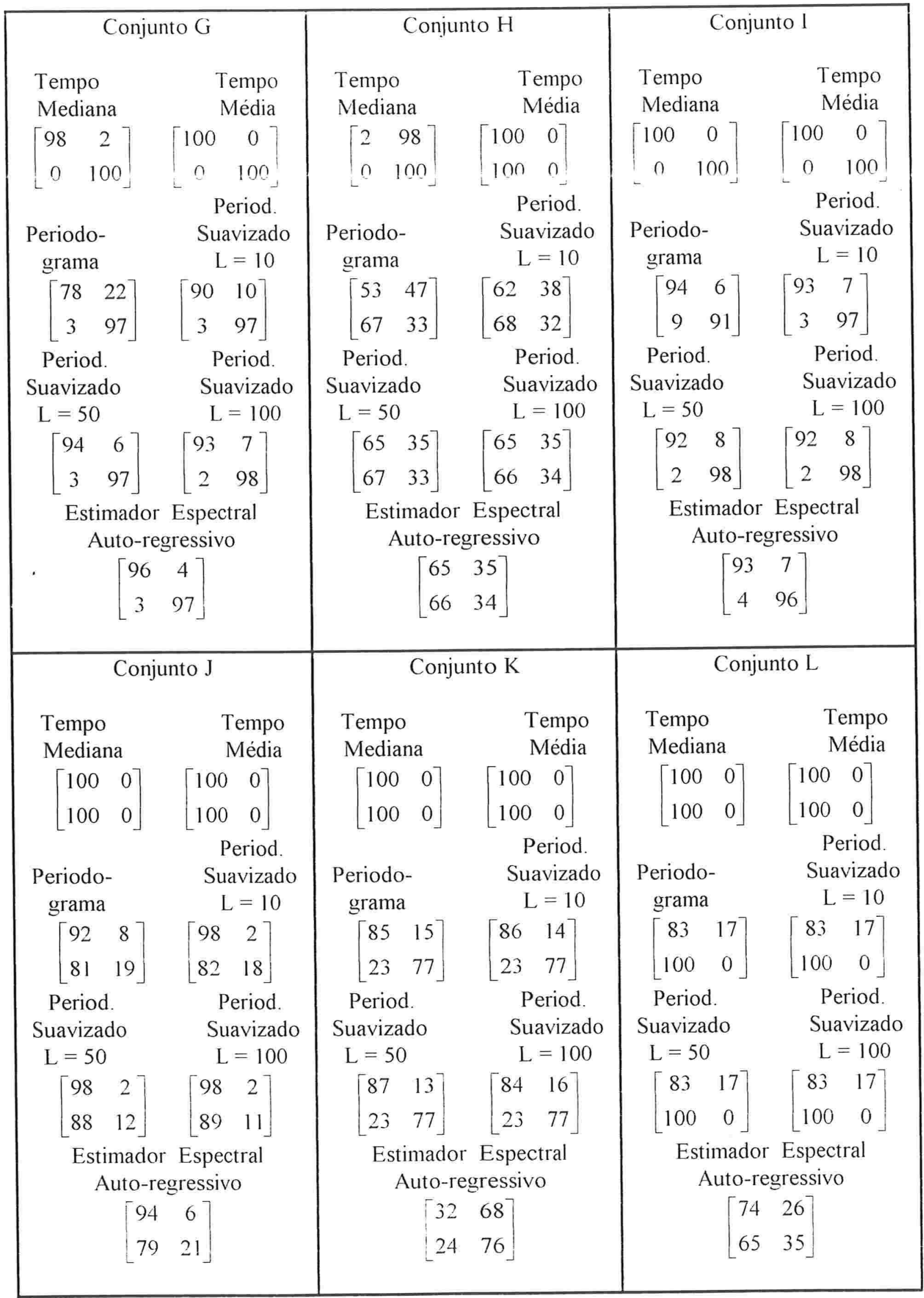

Figura 4.1: Continuação 


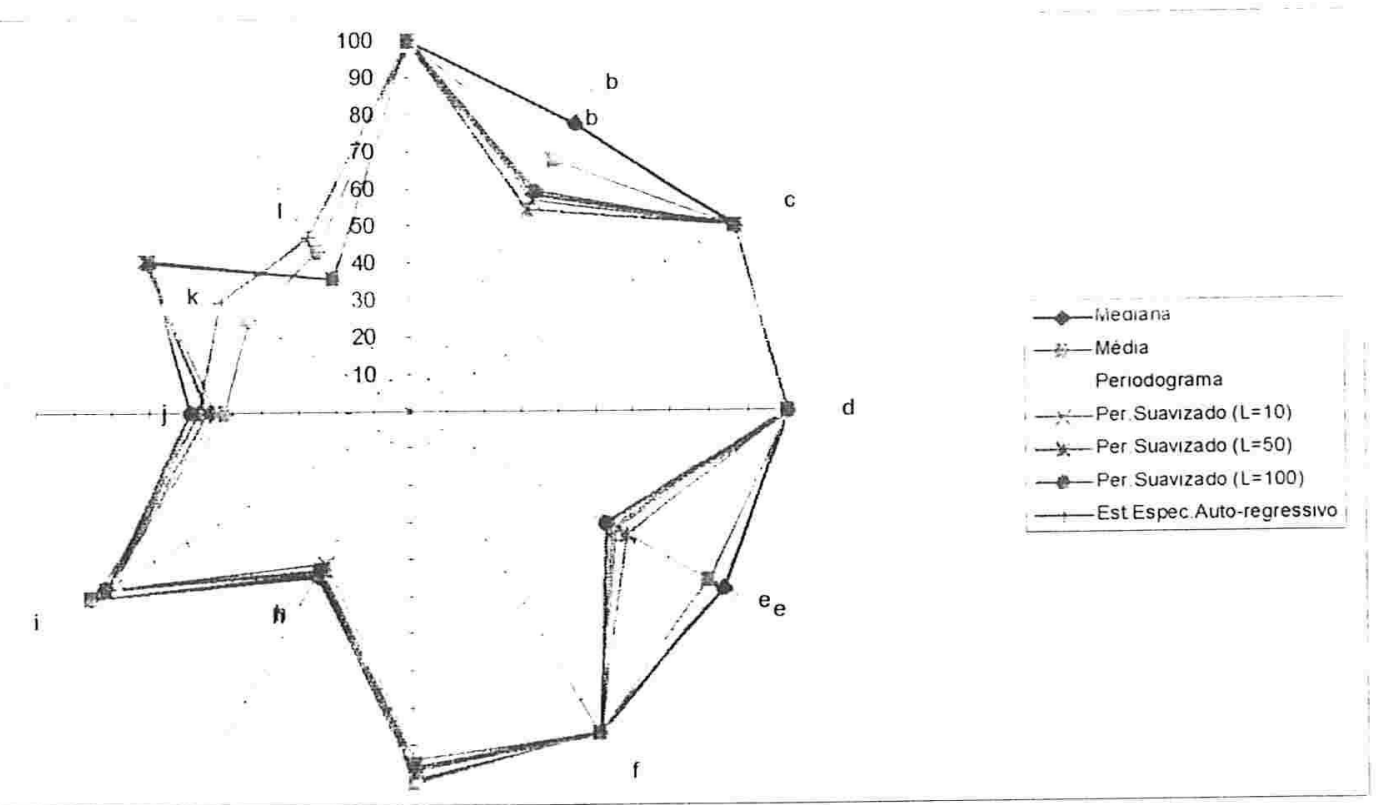

Figura 4.2: (1-Taxa aparente de erro) dos diferentes métodos

Analisando a Tabela 4.2 e a Figura 4.2, verificamos:

a) De uma forma geral, os métodos no domínio da frequência apresentaram desempenho semelhantes e inferiores aos obtidos pelo método no domínio do tempo, exceto nos grupos J e K, cuja inclusão da componente sazonal, propiciou um melhor desempenho para os métodos baseados no domínio da freqüência. O grupo L, diferentemente do esperado, apresentou uma melhor performance do método no domínio do tempo, sendo também onde encontramos os piores resultados (conforme figura 4.2);

b) Dentre os métodos no domínio do tempo, em virtude de sua menor sensibilidade a eventuais valores discrepantes (estimador mais robusto), 
a mediana apresentou uma melhor discriminação do que a média, sendo em alguns casos até $50 \%$ melhor do que este;

c) Dentre os métodos no domínio da freqüência, notamos um desempenho levemente melhor no estimador baseado no periodograma suavisado, utilizando $L=100$ e no estimador espectral auto-regressivo, sendo entretanto, que neste último, diferenças apenas na parte estocática da componente periódica, pouco são percebidas (grupo K);

d) A existência de parâmetros próximos aos limites de não estacionariedade e não invertibilidade não afeta a capacidade de discriminação dos modelos, sendo relevante apenas a distância entre os valores dos parâmetros;

e) Os modelos mais simples $(A R(1), M A(1)$ e $\operatorname{ARMA}(1,1))$ são facilmente discriminados por todos os métodos, quando existe uma diferença razoável no valor de pelo menos um dos parâmetros;

f) Em modelos mais complexos, (por exemplo o $\operatorname{SARIMA}(0,1,1)(0,1,1)_{\mathrm{s}}$ ) a discriminação torna-se bastante complicada, de uma forma geral, se não existe uma diferença razoável entre os valores dos parâmetros. Nestes modelos, a introdução da componente periódica torna os métodos no domínio da freqüência mais competitivos, exceto no grupo L (periodicidades distintas), onde a análise no domínio do tempo 
apresentou uma menor taxa aparente de erro. Entretanto, ao analisarmos mais detalhadamente as matrizes de confusão, averiguamos que os erros concentram-se praticamente na classificação do modelo sazonal de período 9. Dentre os modelos no domínio do tempo, verificamos que, embora o método utilizando-se a mediana apresente melhores resultados em modelos mais simples (sem periodicidade), em modelos mais complexos, seus resultados pouco diferem dos resultados obtidos utilizando-se a média. 


\section{Capítulo 5}

\section{Aplicação a Dados de Eletroencefalogramas}

\subsection{Introdução}

A presente aplicação tem por objeto eletroencefalogramas (EEG) realizados em um grupo de quinze pacientes do Setor de Investigação e Tratamento de Epilepsias, da Escola Paulista de Medicina - SP, que apresentavam EEG alterados na região fronto-temporal esquerda. Para tanto, foram realizadas medições em 20 canais ao longo da caixa craniana, a saber:

F3/F4 e F7/F8 - Frontais

T3/T4 - Temporal anterior

T5/T6 - Temporal médio

T7/T8 - Temporal posterior

P3/P4 - Parietal

01/O2 - Occipital

C3/C4 - Região central

FZ,CZ,PZ e OZ - Linha dos centrais,

separados segundo dois hemisférios: direito e esquerdo. Os canais referentes ao hemisfério esquerdo são numerados segundo uma numeração ímpar, enquanto as do hemisfério direito por numerações pares. Para a coleta das informações, cada indivíduo foi submetido a uma medição em vigilia e submetido a um esforço, que consistia na resolução de uma operação de soma de três algarismos, com os olhos fechados. Após a eliminação de efeitos provocados por elementos alheios 
aos estudados (movimentação ocular, abertura dos olhos, etc), foram identificados padrões que determinassem quais os canais nos quais o paciente apresentava alterações indicativas da epilepsia. Para o presente estudo, foram selecionados individuos que demonstraram problemas nos canais F3, F7, T3 e T5. Desta forma, para cada um dos referidos canais, bem como para seus pares (F4, F8, T4 e T6) selecionou-se períodos de aproximadamente 5,6 segundos (ou seja, 1.000 observações a uma velocidade de captação de 180 pontos por segundo), em períodos cujo comportamento epilético não se mostrava presente. Apesar do comportamento do eletroencefalograma ser muito distinto no período de crise epilética (e portanto facilmente detectada), o mesmo não ocorre durante o período entre crises, no qual, tanto nas regiões normais como nas regiões epiléticas, o padrão do EEG é visualmente semelhante, apresentando apenas pequenas irregularidades no contorno da série. Assim, o objetivo desta aplicação é verificar a capacidade dos diferentes métodos na discriminação destes dois grupos, e por consequência, na detecção de indivíduos que apresentem epilepsia apesar do não diagnóstico nos estudos tradicionais do eletroencefalograma.

As Figuras 5.1 e 5.2 , apresentam as séries dos eletroencefalogramas medidos na região frontal esquerda (onde existe a ocorrência de alterações no exame) e direita (região normal), respectivamente. As Figuras 5.3 e 5.4, de modo análogo, apresentam as séries obtidas na região temporal. 


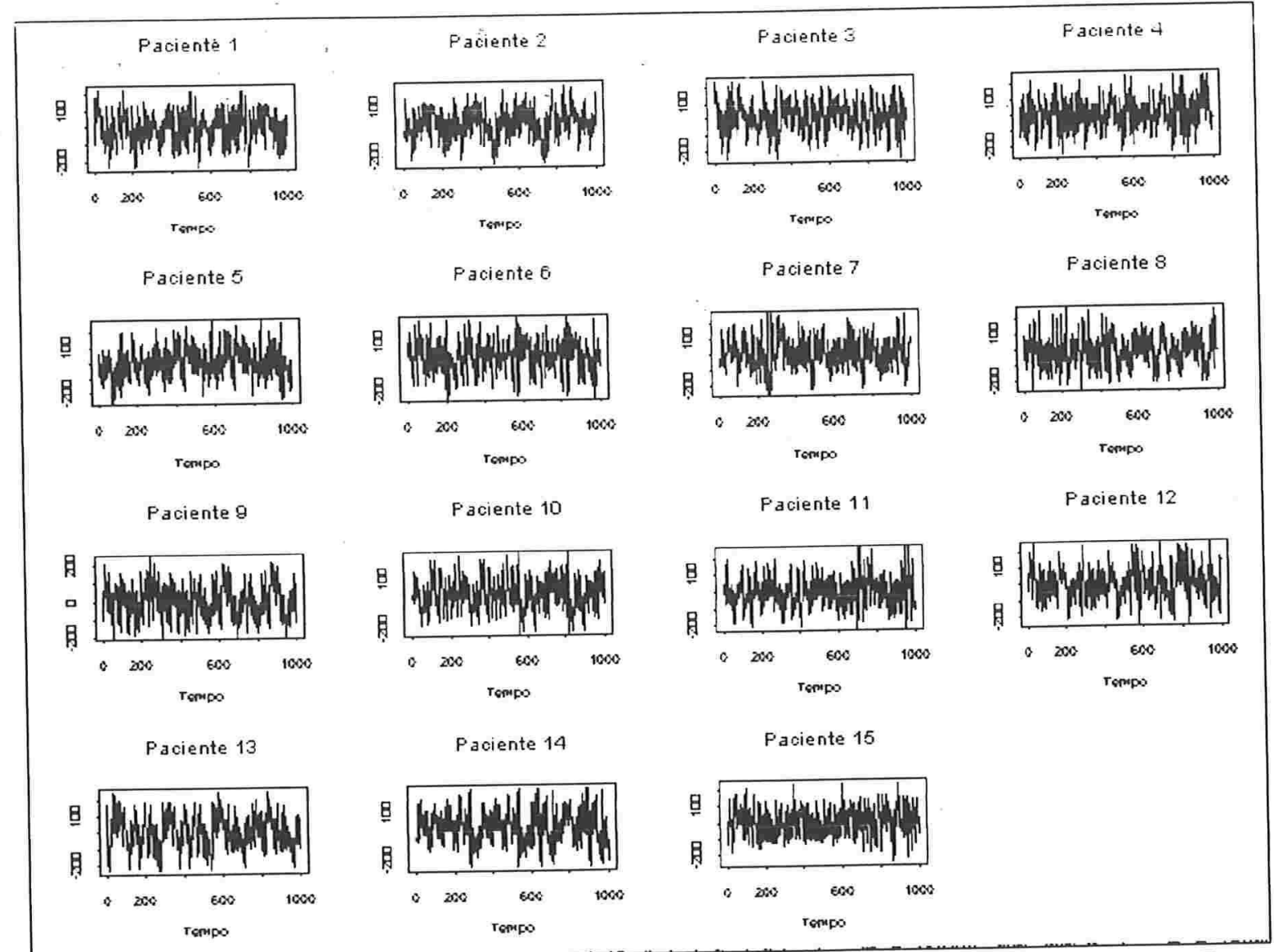

Figura 5.1: Eletroencefalograma da região F3 (frontal - esquerda) de 15 pacientes com sinal alterado 


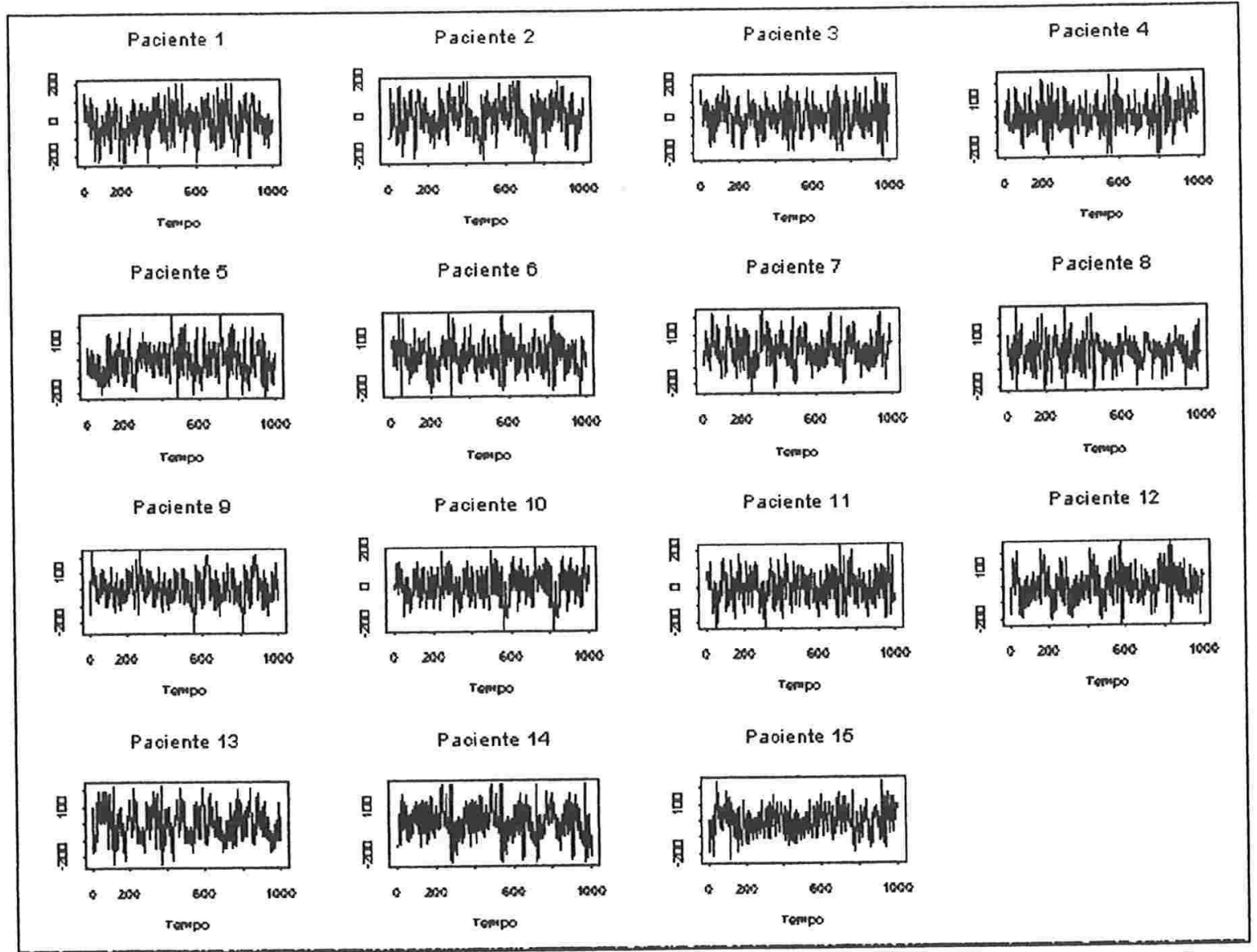

Figura 5.2: Eletroencefalograma da região F4 (frontal - direita) de 15 pacientes com sinal normal 


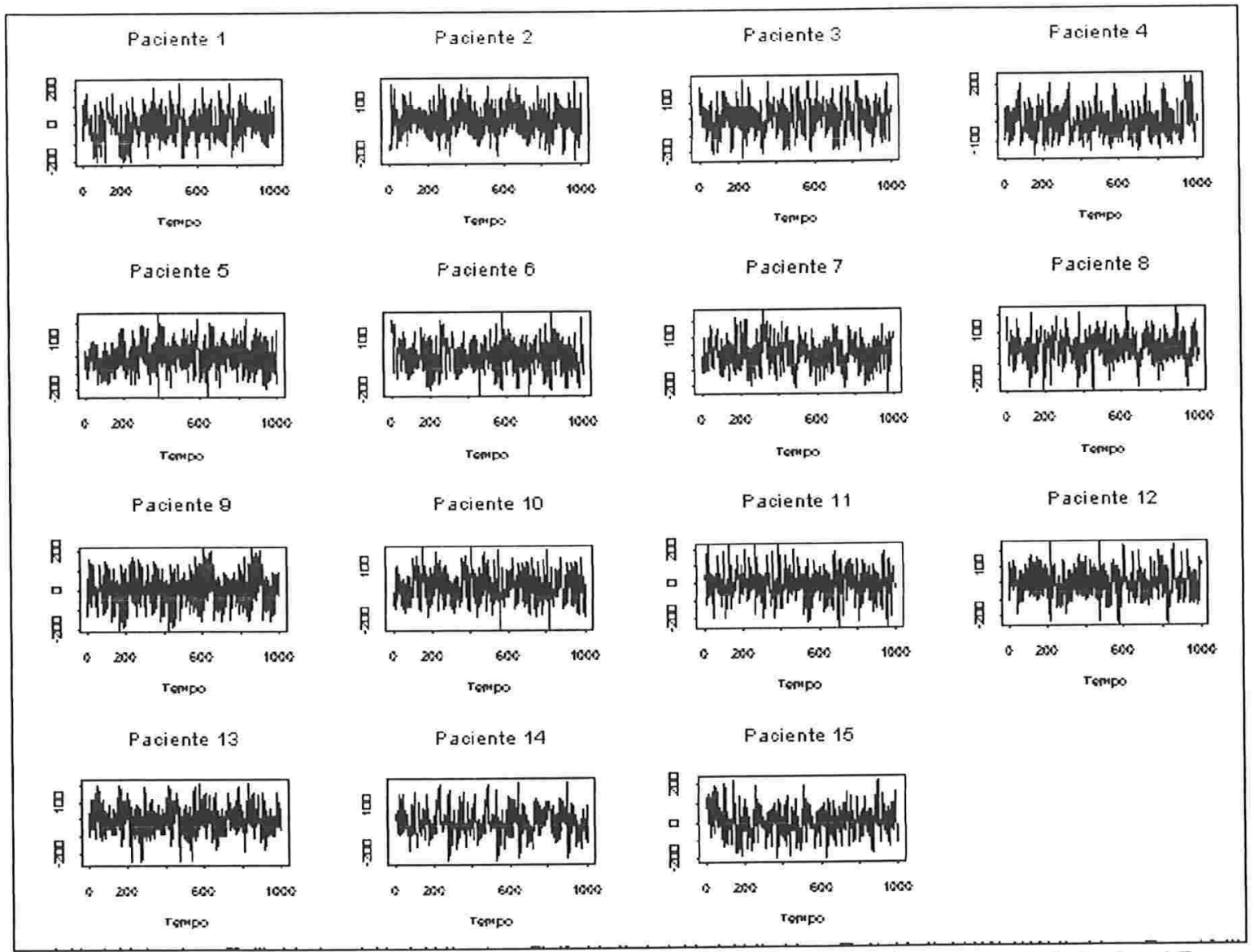

Figura 5.3: Eletroencefalograma da região T3 (Temporal - esquerda) de 15 pacientes com sinal alterado 


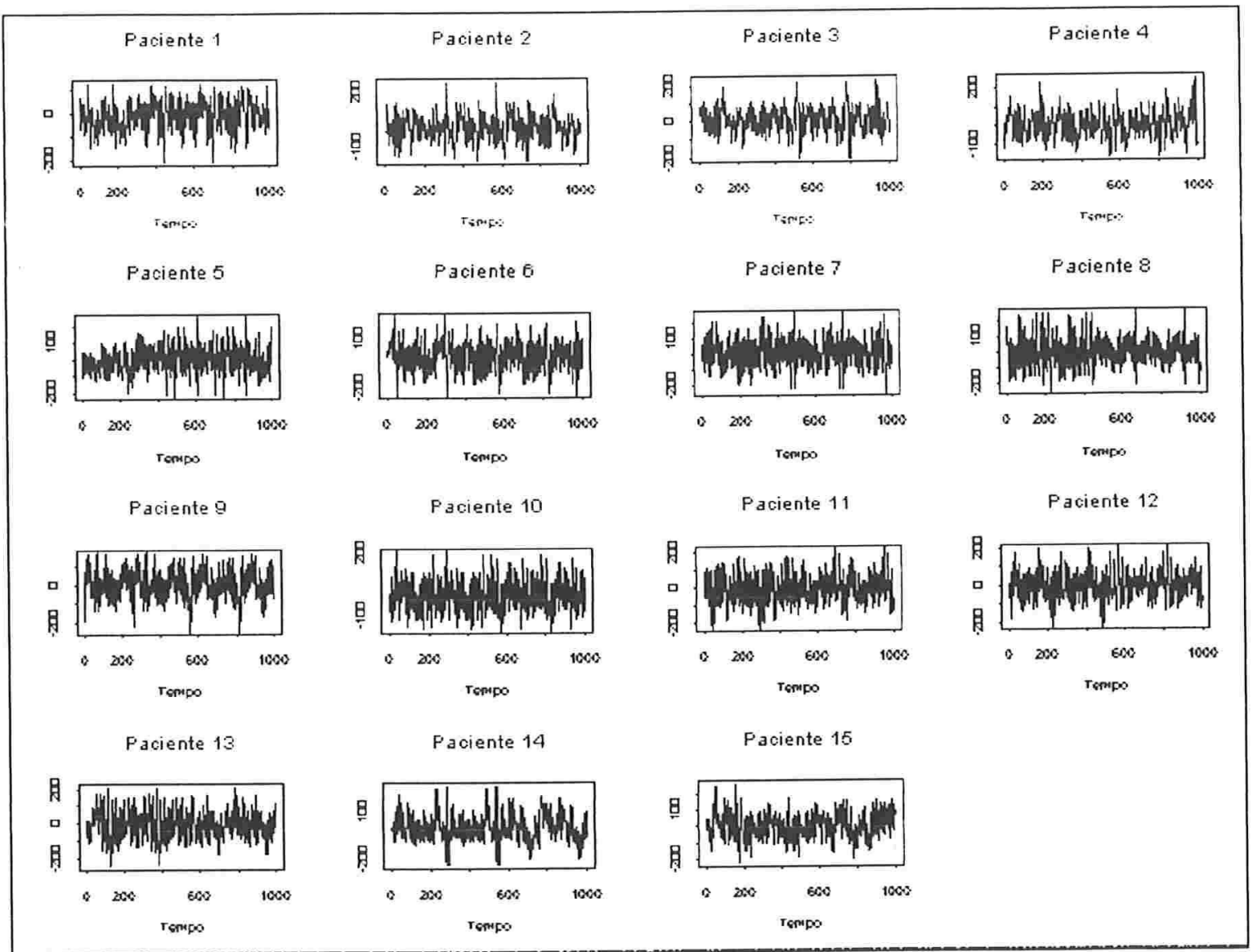

Figura 5.4: Eletroencefalograma da região T4 (Temporal - direita) de 15 pacientes com sinal normal

Aparentemente, a partir da mera observação das séries, torna-se difícil a determinação de padrões que possam identificar um grupo do outro.

A análise dos dados foi realizada utilizando-se o pacote S-Plus (1995). Foram criadas funções especificas para análise discriminante, tanto no domínio do tempo como no domínio da frequência; tais funções encontram-se no Apêndice A. 


\subsection{Análise Discriminante no Domínio do Tempo}

Para a realização da análise discriminante no domínio do tempo, inicialmente, ajustamos modelos auto-regressivos para cada série, a partir do critério AIC de Akaike. Os resultados estão apresentados na Tabela 5.1.

Tabela 5.1 - Ordem dos auto-regressivos obtidos para cada série

\begin{tabular}{|c|c|c|c|c|}
\hline No. & Ordem & Ordem & Ordem & Ordem \\
\hline da & obtida - Grupo & obtida - Grupo & obtida - Grupo & obtida - Grupo \\
\hline Série & $1(F 3)$ & $2(F 4)$ & 3 (T3) & $4(\mathrm{~T} 4)$ \\
\hline 1 & 12 & 23 & 30 & 22 \\
\hline 2 & 19 & 29 & 17 & 21 \\
\hline 3 & 22 & 25 & 13 & 28 \\
\hline 4 & 25 & 18 & 25 & 25 \\
\hline 5 & 28 & 30 & 15 & 28 \\
\hline 6 & 24 & 30 & 23 & 26 \\
\hline 7 & 26 & 30 & 25 & 13 \\
\hline 8 & 22 & 22 & 22 & 28 \\
\hline 9 & 19 & 30 & 29 & 29 \\
\hline 10 & 22 & 30 & 9 & 28 \\
\hline 11 & 20 & 30 & 29 & 30 \\
\hline
\end{tabular}




\begin{tabular}{|c|c|c|c|c|}
\hline No. & Ordem & Ordem & Ordem & Ordem \\
\hline da & obtida - Grupo & obtida - Grupo & obtida - Grupo & obtida - Grupo \\
\hline Série & $1(\mathrm{~F} 3)$ & $2(F 4)$ & $3(\mathrm{~T} 3)$ & $4(\mathrm{~T} 4)$ \\
\hline 12 & 25 & 29 & 17 & 29 \\
\hline 13 & 19 & 19 & 26 & 28 \\
\hline 14 & 25 & 17 & 3 & 9 \\
\hline 15 & 17 & 29 & 23 & 30 \\
\hline Máx. & 28 & 30 & 30 & 30 \\
\hline
\end{tabular}

Posteriormente, ajustamos modelos auto-regressivos utilizando-se a máxima ordem obtida em cada grupo, sendo seus coeficientes apresentados nas Figuras 5.5 a 5.8. 


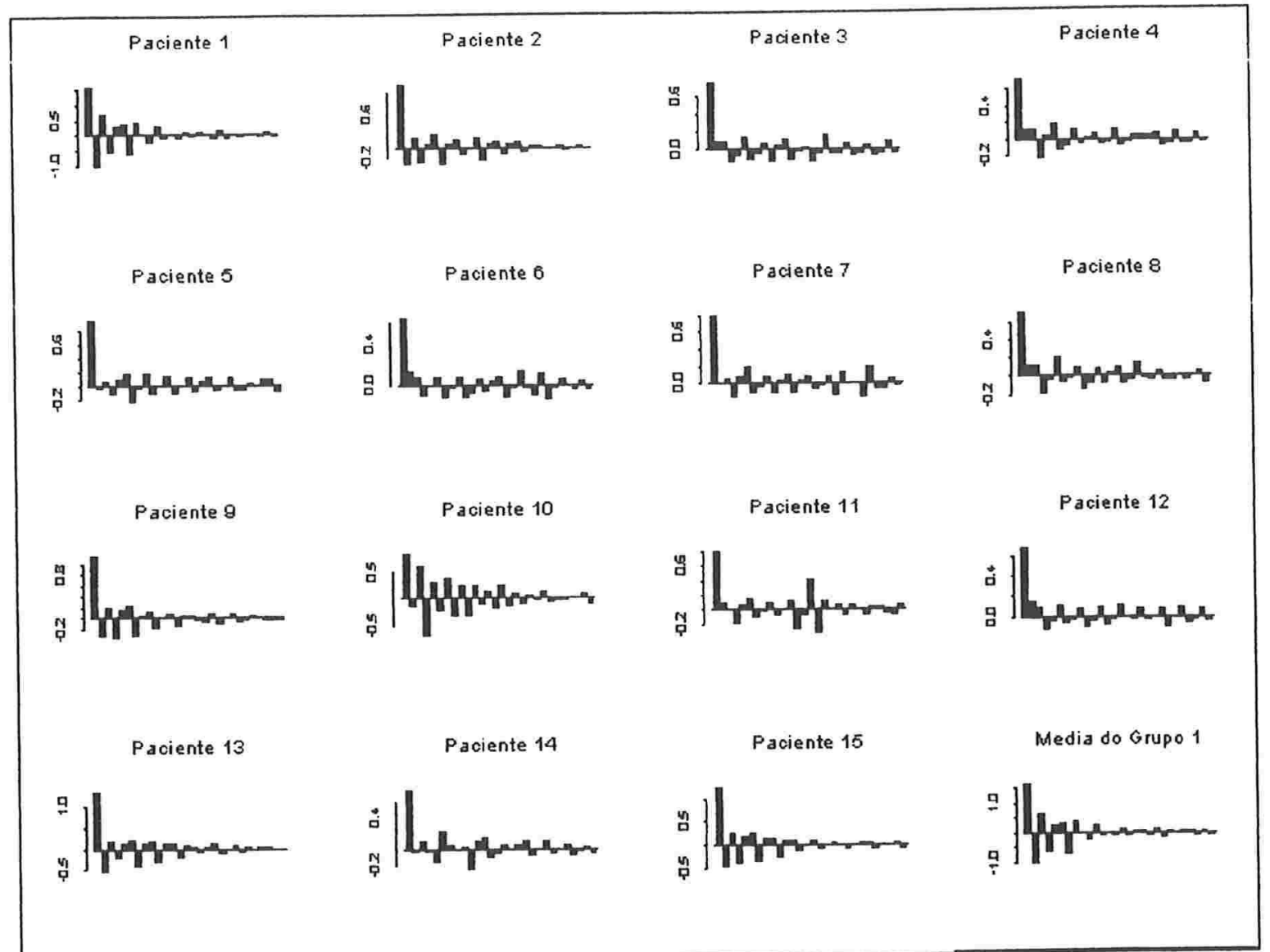

Figura 5.5: Coeficientes dos modelos auto-regressivos obtidos na região F3 (Frontal - esquerda) de 15 pacientes com sinal alterado

Modelo obtido para o grupo 1:

$X(t)=\hat{\phi}_{1} X(t-1)+\hat{\phi}_{2} X(t-2)+\ldots+\hat{\phi}_{28} X(t-28)+a(t)$

onde:

Método utilizando a média:

$\begin{array}{llllll}\hat{\phi}_{1}=1,59 & \hat{\phi}_{2}=-1,03 & \hat{\phi}_{3}=0,65 & \hat{\phi}_{4}=-0,57 & \hat{\phi}_{5}=0,25 & \hat{\phi}_{6}=0,32 \\ \hat{\phi}_{7}=-0,65 & \hat{\phi}_{8}=0,41 & \hat{\phi}_{9}=-0,01 & \hat{\phi}_{10}=-0,22 & \hat{\phi}_{11}=0,27 & \hat{\phi}_{12}=-0,09 \\ \hat{\phi}_{13}=0,01 & \hat{\phi}_{14}=-0,08 & \hat{\phi}_{15}=0,08 & \hat{\phi}_{16}=-0,07 & \hat{\phi}_{17}=0,06 & \hat{\phi}_{18}=0,04 \\ \hat{\phi}_{19}=-0,11 & \hat{\phi}_{20}=0,15 & \hat{\phi}_{21}=-0,12 & \hat{\phi}_{22}=0,02 & \hat{\phi}_{23}=-0,04 & \hat{\phi}_{24}=0,04 \\ \hat{\phi}_{25}=0,05 & \hat{\phi}_{26}=-0,07 & \hat{\phi}_{27}=0,07 & \hat{\phi}_{28}=-0,05 & & \end{array}$

Método utilizando a mediana:

$\begin{array}{llllll}\hat{\phi}_{1}=0,79 & \hat{\phi}_{2}=-0,03 & \hat{\phi}_{3}=0,11 & \hat{\phi}_{4}=-0,19 & \hat{\phi}_{5}=0,06 & \hat{\phi}_{6}=0,18 \\ \hat{\phi}_{7}=-0,11 & \hat{\phi}_{8}=-0,01 & \hat{\phi}_{9}=0,10 & \hat{\phi}_{10}=-0,13 & \hat{\phi}_{11}=0,02 & \hat{\phi}_{12}=0,09 \\ \hat{\phi}_{13}=-0,11 & \hat{\phi}_{14}=-0,01 & \hat{\phi}_{15}=0,09 & \hat{\phi}_{16}=-0,08 & \hat{\phi}_{17}=0,02 & \hat{\phi}_{18}=0,09 \\ \hat{\phi}_{19}=-0,04 & \hat{\phi}_{20}=-0,02 & \hat{\phi}_{21}=0,07 & \hat{\phi}_{22}=-0,05 & \hat{\phi}_{23}=-0,03 & \hat{\phi}_{24}=0,04 \\ \hat{\phi}_{25}=-0,01 & \hat{\phi}_{26}=-0,03 & \hat{\phi}_{27}=0,06 & \hat{\phi}_{28}=-0,04 & & \end{array}$




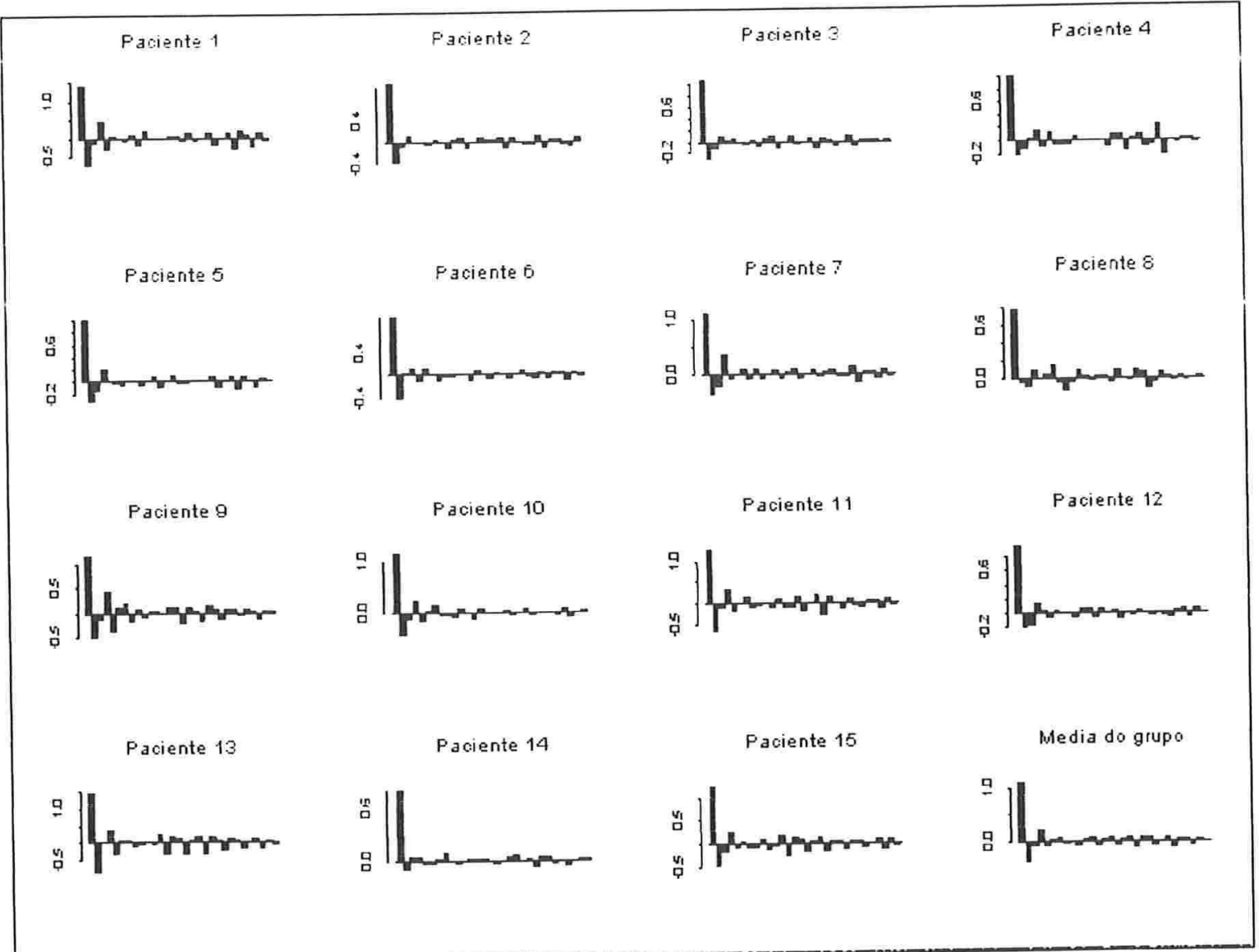

Figura 5.6: Coeficientes dos modelos auto-regressivos obtidos na região $\mathrm{F} 4$ (Frontal - direita) de 15 pacientes com sinal normal

Modelo obtido para o grupo 2:

$X(t)=\hat{\phi}_{1} X(t-1)+\hat{\phi}_{2} X(t-2)+\ldots+\hat{\phi}_{30} X(t-30)+a(t)$

onde:

Método utilizando a média:

$\begin{array}{llllll}\hat{\phi}_{1}=1,21 & \hat{\phi}_{2}=-0,45 & \hat{\phi}_{3}=-0,04 & \hat{\phi}_{4}=0,17 & \hat{\phi}_{5}=-0,05 & \hat{\phi}_{6}=0,05 \\ \hat{\phi}_{7}=-0,04 & \hat{\phi}_{8}=-0,04 & \hat{\phi}_{9}=0,11 & \hat{\phi}_{10}=-0,13 & \hat{\phi}_{11}=0,01 & \hat{\phi}_{12}=0,10 \\ \hat{\phi}_{13}=-0,16 & \hat{\phi}_{14}=0,08 & \hat{\phi}_{15}=0,09 & \hat{\phi}_{16}=-0,13 & \hat{\phi}_{17}=0,03 & \hat{\phi}_{18}=0,14 \\ \hat{\phi}_{19}=-0,16 & \hat{\phi}_{20}=0,06 & \hat{\phi}_{21}=0,10 & \hat{\phi}_{22}=-0,16 & \hat{\phi}_{23}=0,07 & \hat{\phi}_{24}=0,07 \\ \hat{\phi}_{25}=-0,13 & \hat{\phi}_{26}=0,05 & \hat{\phi}_{27}=0,07 & \hat{\phi}_{28}=-0,14 & \hat{\phi}_{29}=0,08 & \hat{\phi}_{30}=-0,03\end{array}$

Método utilizando a mediana:

$\begin{array}{llllll}\hat{\phi}_{1}=1,31 & \hat{\phi}_{2}=-0,50 & \hat{\phi}_{3}=-0,07 & \hat{\phi}_{4}=0,24 & \hat{\phi}_{5}=-0,09 & \hat{\phi}_{6}=0,03 \\ \hat{\phi}_{7}=-0,07 & \hat{\phi}_{8}=-0,02 & \hat{\phi}_{9}=0,11 & \hat{\phi}_{10}=-0,14 & \hat{\phi}_{11}=0,01 & \hat{\phi}_{12}=0,08 \\ \hat{\phi}_{13}=-0,15 & \hat{\phi}_{1 \cdot 1}=0,04 & \hat{\phi}_{15}=0,10 & \hat{\phi}_{16}=-0,13 & \hat{\phi}_{17}=0,03 & \hat{\phi}_{18}=0,16 \\ \hat{\phi}_{19}=-0,17 & \hat{\phi}_{20}=0,05 & \hat{\phi}_{21}=0,09 & \hat{\phi}_{22}=-0,13 & \hat{\phi}_{23}=0,06 & \hat{\phi}_{24}=0,08 \\ \hat{\phi}_{25}=-0,13 & \hat{\phi}_{20}=0,01 & \hat{\phi}_{27}=0,09 & \hat{\phi}_{28}=-0,13 & \hat{\phi}_{29}=0,08 & \hat{\phi}_{30}=-0,02\end{array}$




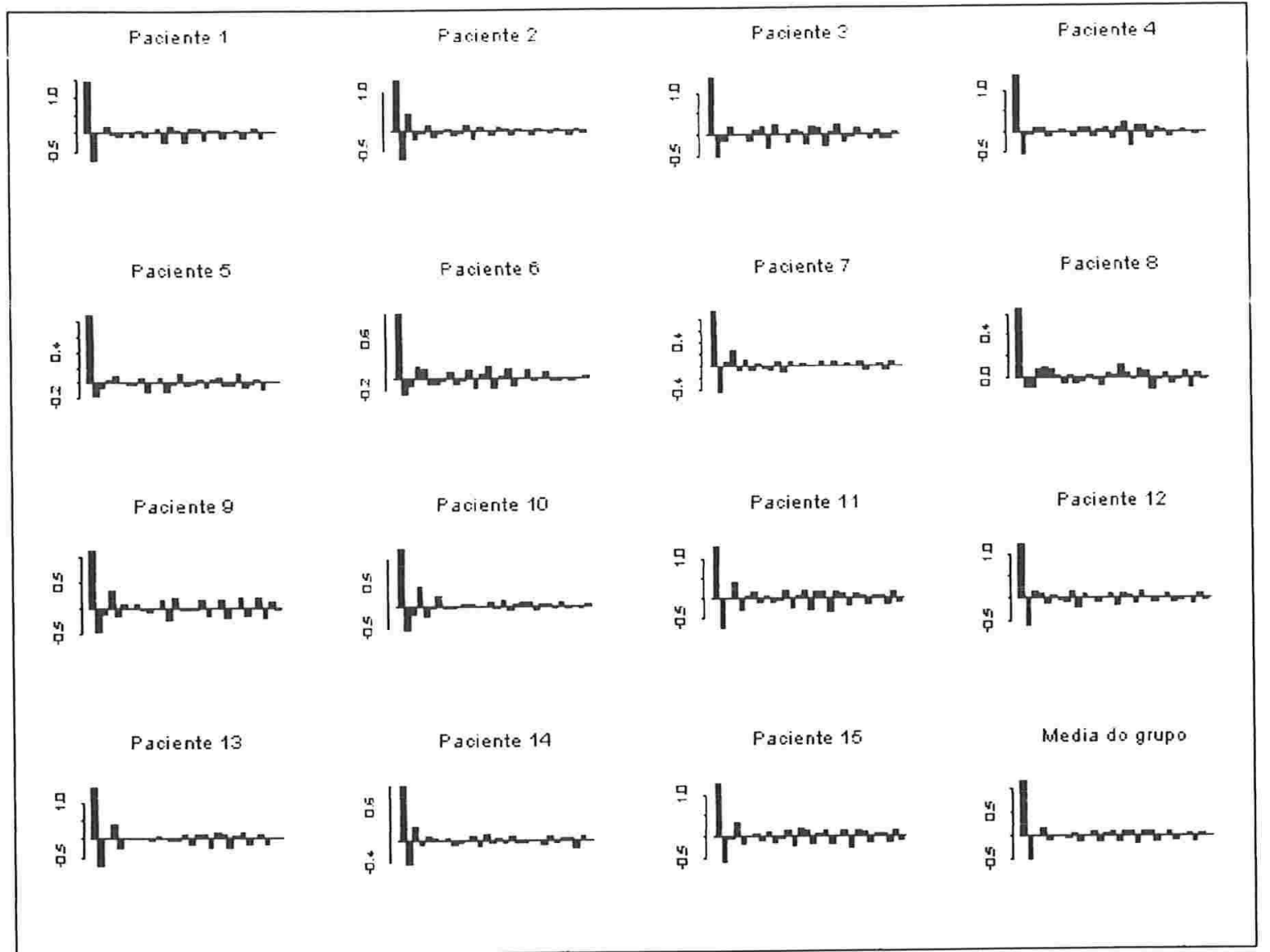

Figura 5.7: Coeficientes dos modelos auto-regressivos obtidos na região T3 (Temporal - esquerda) de 15 pacientes com sinal alterado

Modelo obtido para o grupo 3:

$X(t)=\hat{\phi}_{1} X(t-1)+\hat{\phi}_{2} X(t-2)+\ldots+\hat{\phi}_{30} X(t-30)+a(t)$

onde:

Método utilizando a média:

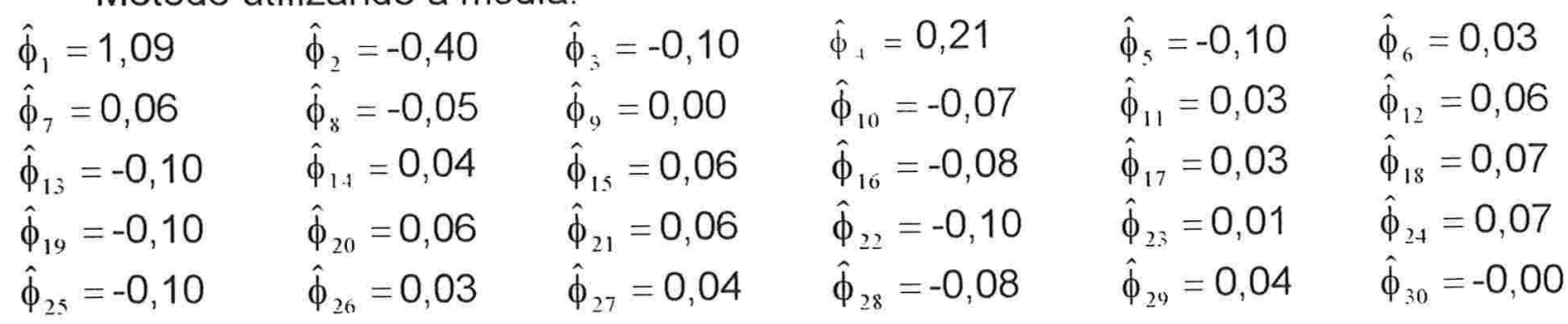

Método utilizando a mediana:

$\begin{array}{llllll}\hat{\phi}_{1}=1,07 & \hat{\phi}_{2}=-0,37 & \hat{\phi}_{3}=-0,11 & \hat{\phi}_{1}=0,20 & \hat{\phi}_{3}=-0,09 & \hat{\phi}_{0}=0,04 \\ \hat{\phi}_{7}=0,02 & \hat{\phi}_{8}=-0,05 & \hat{\phi}_{9}=0,00 & \hat{\phi}_{10}=-0,07 & \hat{\phi}_{11}=0,03 & \hat{\phi}_{12}=0,06 \\ \hat{\phi}_{13}=-0,08 & \hat{\phi}_{14}=0,02 & \hat{\phi}_{15}=0,06 & \hat{\phi}_{16}=-0,04 & \hat{\phi}_{17}=0,03 & \hat{\phi}_{18}=0,05 \\ \hat{\phi}_{19}=-0,06 & \hat{\phi}_{20}=0,04 & \hat{\phi}_{21}=0,06 & \hat{\phi}_{22}=-0,08 & \hat{\phi}_{23}=0,00 & \hat{\phi}_{24}=0,06 \\ \hat{\phi}_{25}=-0,08 & \hat{\phi}_{26}=0,02 & \hat{\phi}_{27}=0,04 & \hat{\phi}_{28}=-0,08 & \hat{\phi}_{29}=0,04 & \hat{\phi}_{30}=0,00\end{array}$




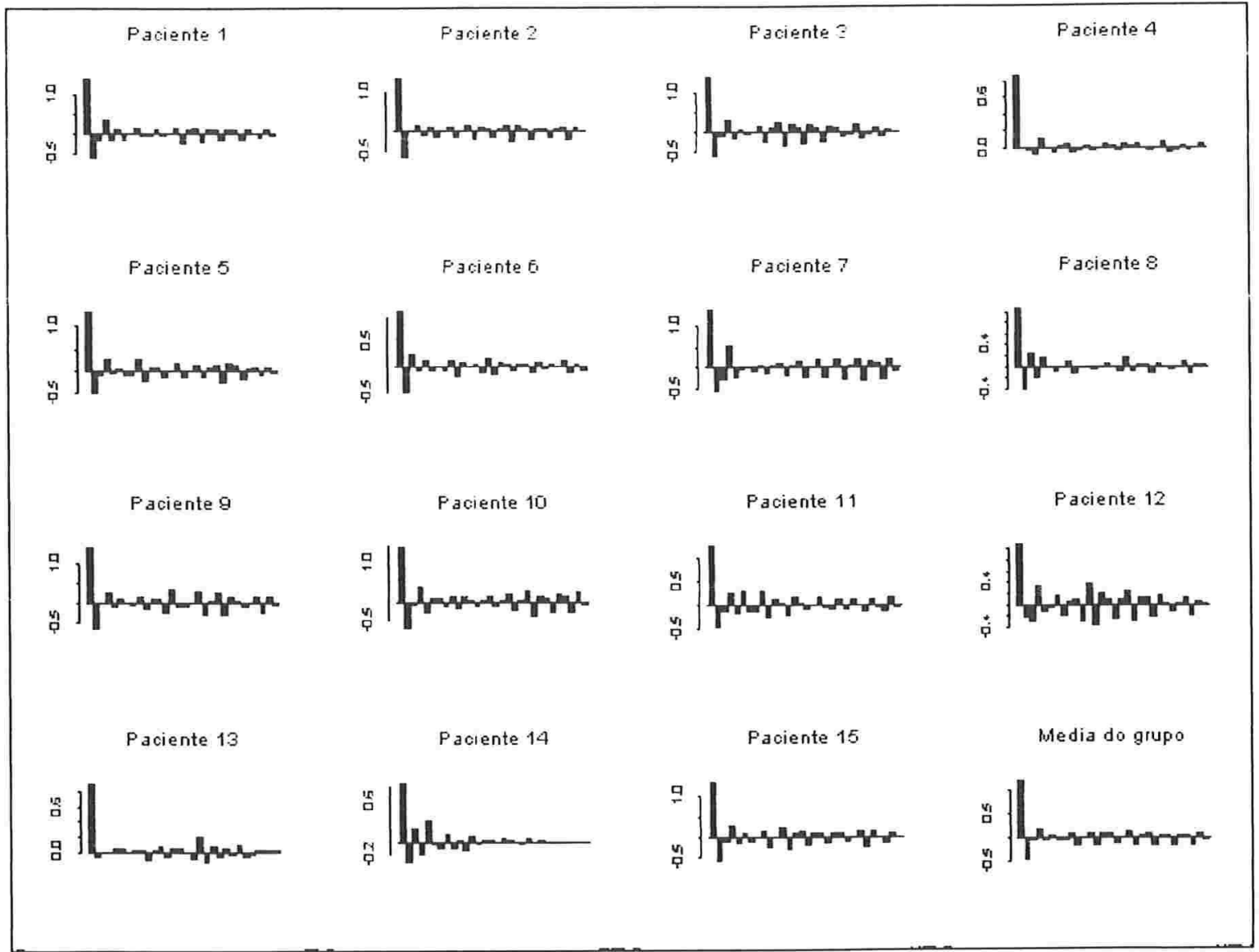

Figura 5.8: Coeficientes dos modelos auto-regressivos obtidos na região T4 (Temporal - direita) de 15 pacientes com sinal normal

Modelo obtido para o grupo 4:

$X(t)=\hat{\phi}_{1} X(t-1)+\hat{\phi}_{2} X(t-2)+\ldots+\hat{\phi}_{30} X(t-30)+a(t)$

onde:

Método utilizando a média:

$\begin{array}{llllll}\hat{\phi}_{1}=1,16 & \hat{\phi}_{2}=-0,50 & \hat{\phi}_{3}=0,00 & \hat{\phi}_{4}=0,19 & \hat{\phi}_{5}=-0,06 & \hat{\phi}_{6}=0,02 \\ \hat{\phi}_{7}=0,00 & \hat{\phi}_{8}=-0,02 & \hat{\phi}_{9}=0,05 & \hat{\phi}_{10}=-0,10 & \hat{\phi}_{11}=0,02 & \hat{\phi}_{12}=0,07 \\ \hat{\phi}_{13}=-0,13 & \hat{\phi}_{14}=0,06 & \hat{\phi}_{15}=0,07 & \hat{\phi}_{16}=-0,12 & \hat{\phi}_{17}=0,07 & \hat{\phi}_{18}=0,10 \\ \hat{\phi}_{19}=-0,15 & \hat{\phi}_{20}=0,07 & \hat{\phi}_{21}=0,08 & \hat{\phi}_{22}=-0,12 & \hat{\phi}_{23}=0,02 & \hat{\phi}_{24}=0,08 \\ \hat{\phi}_{25}=-0,09 & \hat{\phi}_{26}=0,01 & \hat{\phi}_{27}=0,05 & \hat{\phi}_{28}=-0,10 & \hat{\phi}_{29}=0,04 & \hat{\phi}_{30}=0,01\end{array}$

Método utilizando a mediana:

$\begin{array}{llllll}\hat{\phi}_{1}=1,22 & \hat{\phi}_{2}=-0,53 & \hat{\phi}_{3}=0,02 & \hat{\phi}_{1}=0,19 & \hat{\phi}_{5}=-0,05 & \hat{\phi}_{6}=0,02 \\ \hat{\phi}_{7}=0,00 & \hat{\phi}_{8}=-0,03 & \hat{\phi}_{13}=0,06 & \hat{\phi}_{10}=-0,08 & \hat{\phi}_{11}=0,00 & \hat{\phi}_{12}=0,07 \\ \hat{\phi}_{13}=-0,13 & \hat{\phi}_{1-1}=0,05 & \hat{\phi}_{15}=0,08 & \hat{\phi}_{16}=-0,15 & \hat{\phi}_{17}=0,08 & \hat{\phi}_{18}=0,11 \\ \hat{\phi}_{19}=-0,14 & \hat{\phi}_{20}=0,07 & \hat{\phi}_{21}=0,09 & \hat{\phi}_{22}=-0,12 & \hat{\phi}_{23}=0,02 & \hat{\phi}_{24}=0,07 \\ \hat{\phi}_{25}=-0,08 & \hat{\phi}_{26}=0,01 & \hat{\phi}_{2-2}=0,05 & \hat{\phi}_{28}=-0,10 & \hat{\phi}_{29}=0,04 & \hat{\phi}_{30}=0,01\end{array}$


A partir dos modelos anteriormente apresentados. notamos que, com exceção do grupo de séries obtidas na região F3, para os quais observamos uma redução da magnitude dos seus coeficientes de forma mais lenta, os demais apresentam um padrão de comportamento muito semelhante, possuindo os maiores coeficientes nos lags até quatro. Além deste fato, verifica-se também uma leve tendência dos modelos associados as regiões normais possuírem, exporadicamente, coeficientes de magnitude superior aos de seus vizinhos, podendo indicar alguma periodicidade na série.

Para a construção da matriz de confusão, inicialmente obtemos, através da filtragem da série $X(t)$, a ser classificada, os resíduos $\hat{a}_{i}(t)$ e $\hat{\sigma}_{a_{i}}^{2}$ da sequinte forma:

$$
\begin{aligned}
& \hat{a}_{i}(t)=X(t)-\sum_{k=1}^{K_{i}} \bar{\pi}_{k}^{i} X(t-k), t=0,1, \ldots, 999 ; i=1, \ldots, 4 \\
& \hat{\sigma}_{i^{a}}^{2}=\left(T-K_{i}\right)^{-1} \sum_{i=K_{i}}^{T-1} \hat{a}_{i}^{2}(t)
\end{aligned}
$$

Substituindo (5.1) e (5.2) em (3.9), obtemos $\hat{h}_{i}(\mathbf{X}), i=1, . .4$ para cada série da amostra de treinamento. Para a primeira série da amostra de treinamento, supondo probabilidades a priori idênticas, obtivemos os seguintes valores:

$$
\begin{aligned}
& \hat{\mathrm{h}}_{1}(\mathbf{X})=-\frac{(1000-28)}{2} \ln \left(\hat{\sigma}_{\mathrm{a}_{1}}^{2}\right)-\frac{1}{2 \hat{\sigma}_{\mathrm{a}_{t}}^{2}} \sum_{\mathrm{t}=\mathrm{p}-1}^{\mathrm{T}-1} \hat{\mathrm{a}}_{\mathrm{i}}^{2}(\mathrm{t})+\ln 0,25=-3596.565 \\
& \hat{\mathrm{h}}_{2}(\mathbf{X})=-\frac{(1000-28)}{2} \ln \left(\hat{\sigma}_{\mathrm{a}_{1}}^{2}\right)-\frac{1}{2 \hat{\sigma}_{\mathrm{a}_{1}}^{2}} \sum_{\mathrm{t}=\mathrm{p}-1}^{\mathrm{T}-1} \hat{\mathrm{a}}_{\mathrm{i}}^{2}(\mathrm{t})+\ln 0,25=-3898.14 \\
& \hat{\mathrm{h}}_{3}(\mathbf{X})=-\frac{(1000-28)}{2} \ln \left(\hat{\sigma}_{\mathrm{a}_{1}}^{2}\right)-\frac{1}{2 \hat{\sigma}_{\mathrm{a}_{1}}^{2}} \sum_{\mathrm{t}=\mathrm{p}-1}^{\mathrm{T}-1} \hat{\mathrm{a}}_{\mathrm{i}}^{2}(\mathrm{t})+\ln 0.25=-4213.021
\end{aligned}
$$




$$
\hat{h}_{4}(X)=-\frac{(1000-28)}{2} \ln \left(\hat{\sigma}_{\mathrm{s}_{i}}^{2}\right)-\frac{1}{2 \hat{\sigma}_{\mathbf{s}_{i}}^{2}} \sum_{t=p+1}^{\mathrm{T}-1} \hat{\hat{a}}_{i}^{2}(\mathrm{t})+\ln 0,25=-4045.504
$$

Portanto, como $\hat{\mathrm{h}}_{1}(\mathbf{X})$ apresentou o maior valor dentre as demais (-596.565), classificaremos a primeira série como sendo uma observação da região Frontal esquerda (F3). Procedendo de forma análoga para as demais séries,construímos a matriz de confusão, dada pela Tabela 5.2.

Tabela 5.2:Matriz de confusão para o modelo discriminante no dominio do tempo - Média

\begin{tabular}{|c|c|c|c|c|c|}
\hline \multicolumn{2}{|c|}{} & \multicolumn{5}{|c|}{ Classificação Prevista } \\
\cline { 2 - 6 } \multicolumn{2}{|c|}{} & F3 & F4 & T3 & T4 \\
\hline \multirow{3}{*}{$\begin{array}{c}\text { Classificação } \\
\text { Real }\end{array}$} & F3 & $\mathbf{5}$ & 10 & 0 & 0 \\
\cline { 2 - 6 } & F4 & 0 & $\mathbf{1 1}$ & 3 & 1 \\
\cline { 2 - 6 } & T3 & 0 & 3 & 12 & 0 \\
\cline { 2 - 6 } & T4 & 0 & 5 & 5 & $\mathbf{5}$ \\
\hline
\end{tabular}

Utilizando as informações da Tabela 5.2, temos que TAE $=\frac{27}{60}=0,45$, ou seja, este modelo, para o conjunto de dados em questão, apresenta um taxa de erro de $45 \%$, sendo portanto não recomendado. Observando os erros cometidos, notamos uma incapacidade de discriminação tanto entre as regiões como entre os casos normais e epiléticos.

Utilizando-se a mediana em lugar da média, apesar de obtermos melhores resultados, conforme tabela 5.3, a taxa aparente de erro continua elevada (TAE $=0,32)$, indicando a necessidade de utilização de outros métodos. 
Tabela 5.3:Matriz de confusão para o modelo discriminante no domínio do tempo Mediana

\begin{tabular}{|c|c|c|c|c|c|}
\hline \multicolumn{2}{|c|}{} & \multicolumn{4}{|c|}{ Classificação Prevista } \\
\cline { 2 - 6 } \multicolumn{2}{|c|}{} & F3 & F4 & T3 & T4 \\
\hline \multirow{4}{*}{$\begin{array}{c}\text { Classificação } \\
\text { Real }\end{array}$} & F3 & 15 & 0 & 0 & 0 \\
\cline { 2 - 6 } & T3 & 4 & 9 & 0 & 2 \\
\cline { 2 - 6 } & T4 & 0 & 0 & 10 & 4 \\
\hline
\end{tabular}




\subsection{Análise Discriminante no Domínio da Frequência}

Tendo em vista o baixo desempenho do procedimento anterior, refaremos a análise no domínio da frequência. Para tanto, calculamos a transformada de Fourier e o periodograma para cada série e, após a determinação da média das transformadas (conforme (3.12)), calculamos o periodograma médio. Os resultados são apresentados nas Figuras 5.9 a 5.12 , onde podemos notar no periodograma médio das regiões F3 e T3, valores das ordenadas com uma magnitude superior aos obtidos nos grupos normais, indicando a existência de uma maior variabilidade nos grupos epiléticos. Além deste fato, no grupo F3 (Frontal esquerdo) observamos, nas frequências de $0,334 \mathrm{~Hz}$ e $0,668 \mathrm{~Hz}$, valores elevados, indicando a existência de periodicidades de 60 (180 pontos/segundo * freq.) e 120 pontos. Tais periodicidades, embora comuns na rede elétrica do Estado de São Paulo (a Eletropaulo trabalha com uma corrente alternada de 60 ciclos/s), não descarta sua importância, visto que os demais grupos, também sujeitos às mesmas condições, apresentaram valores para esta frequência relativamente baixos. 


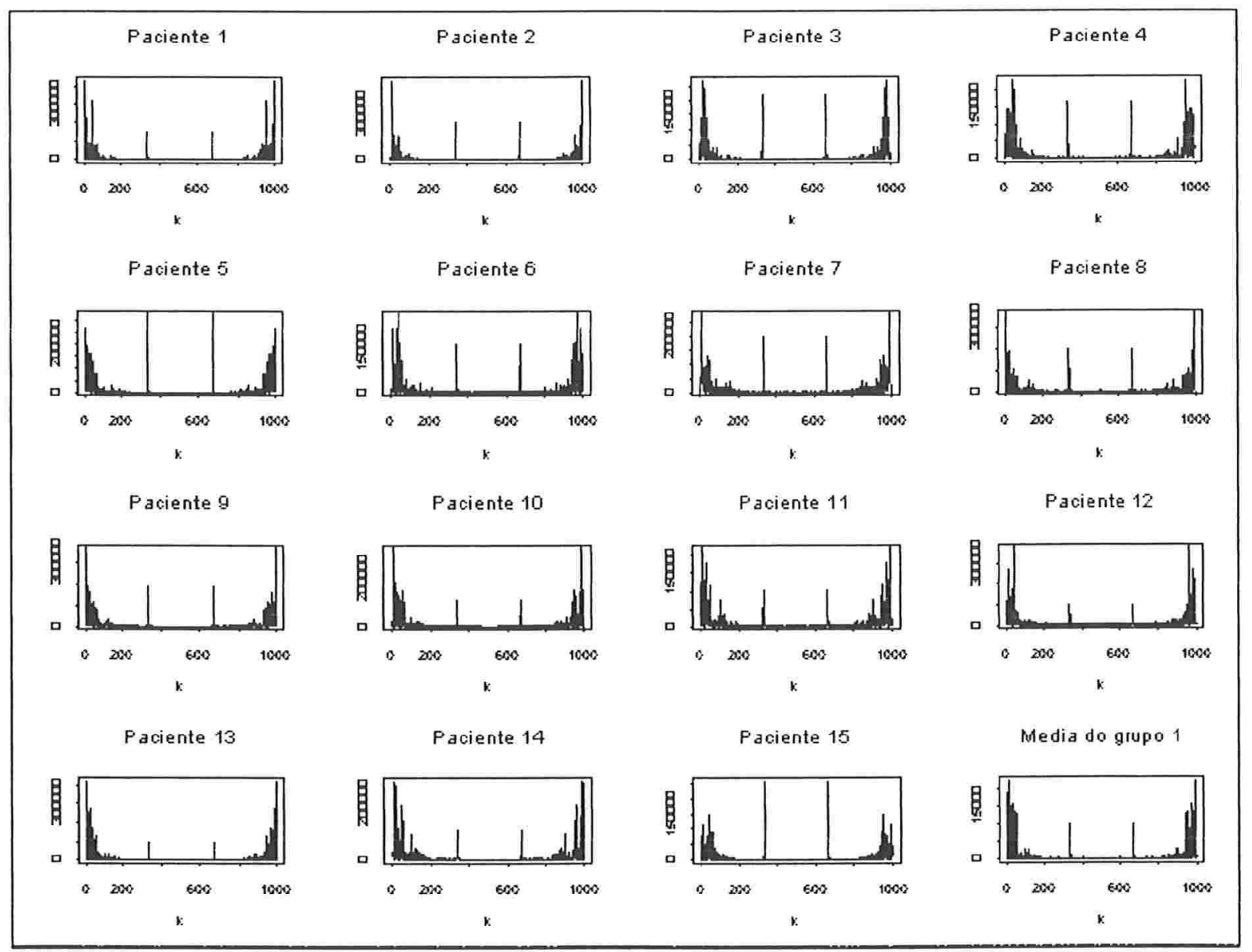

Figura 5.9: Periodograma do eletroencefalograma da região F3 (Frontal esquerda) de 15 pacientes com sinal alterado 


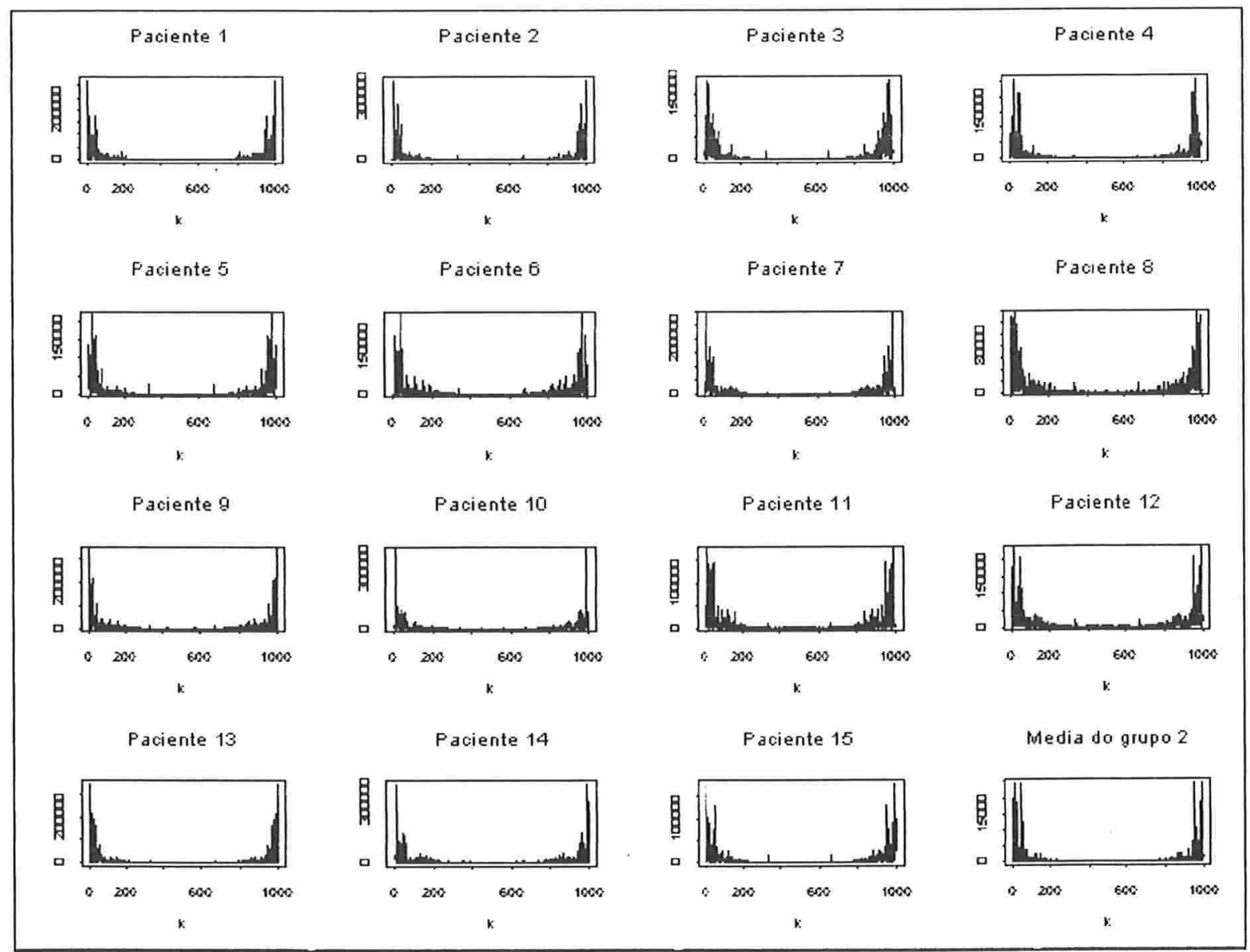

Figura 5.10: Periodograma do eletroencefalograma da região F4 (Frontal direita) de 15 pacientes com sinal normal 


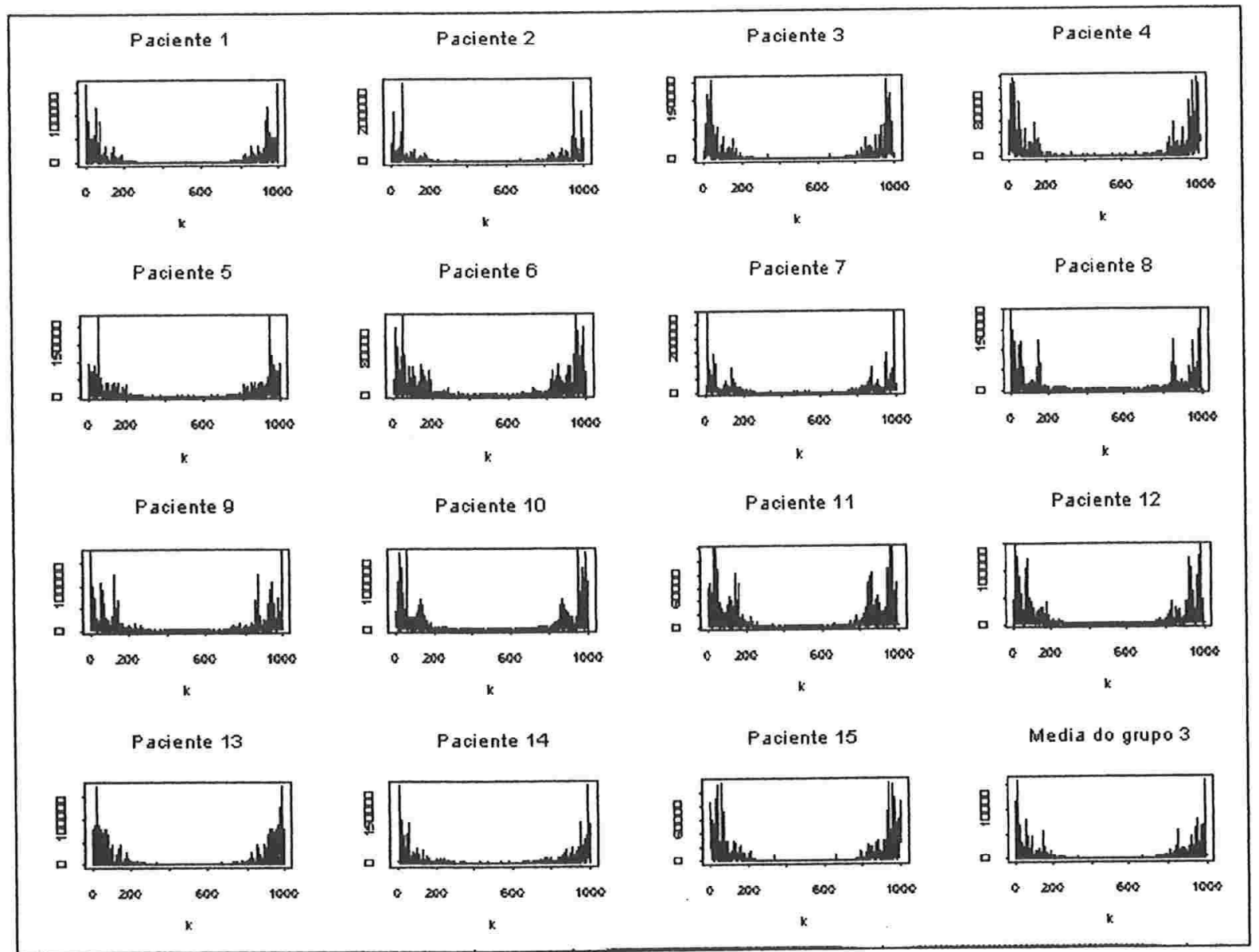

Figura 5.11: Periodograma do eletroencefalograma da região T3 (Temporal esquerda) de 15 pacientes com sinal alterado 


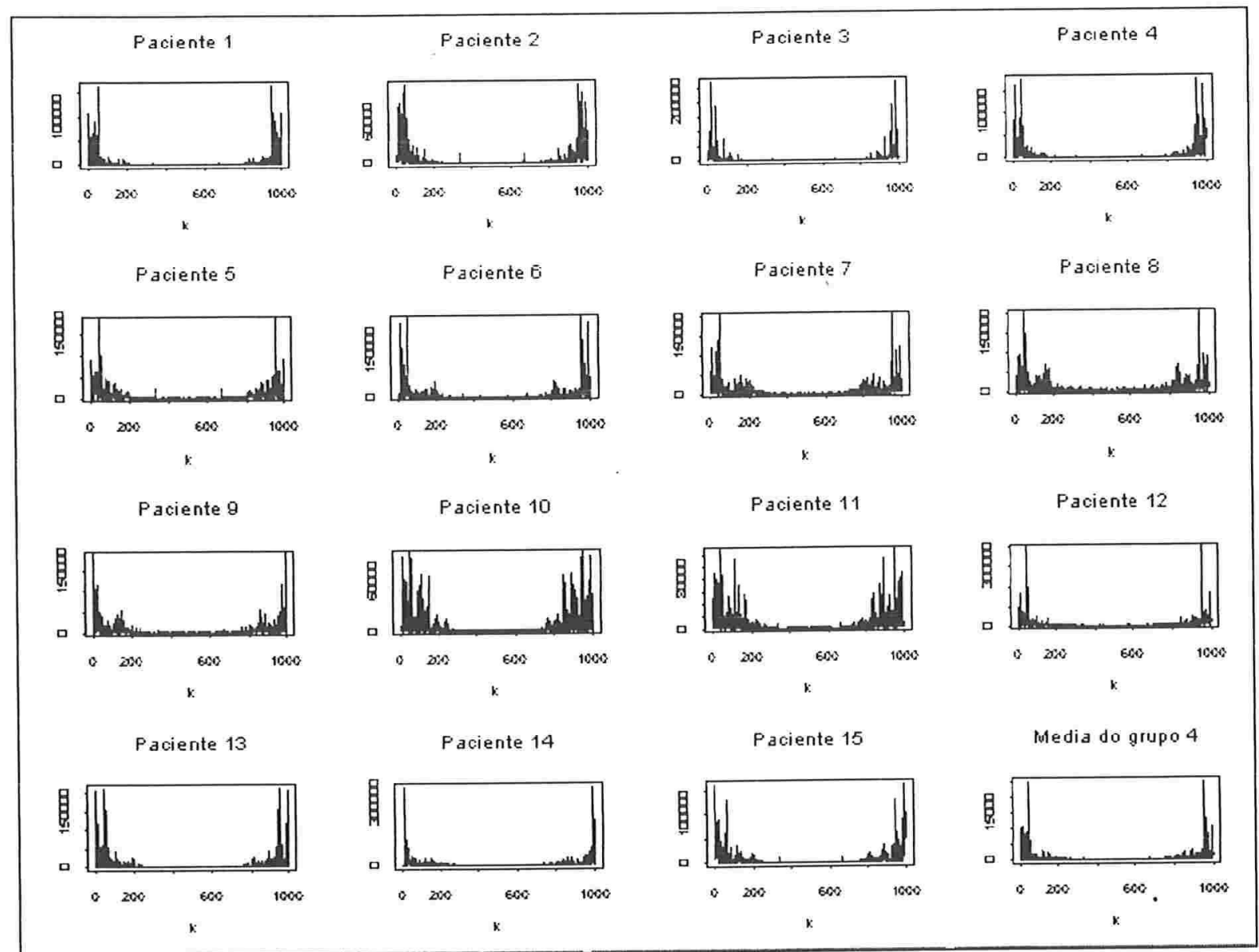

Figura 5.12: Periodograma do eletroencefalograma da região T4 (Temporal direita) de 15 pacientes com sinal normal

Apresentamos nas Figuras 5.13 a 5.16 , os estimadores espectrais autoregressivos para cada série. Para o cálculo do estimador espectral autoregressivo de cada grupo, escolhemos a maior ordem $\left(\mathrm{K}_{\mathrm{i}}\right)$ obtida dentre os modelos auto-regressivos de cada série (obtidos a partir do critério AIC de Akaike) e posteriormente reestimamos os parâmetros impondo um modelo $A R\left(K_{i}\right)$ para cada grupo. Em seguida, calculando-se a média dos parâmetros estimados em cada grupo e utilizando-se (2.40), determinamos o estimador espectral médio. 

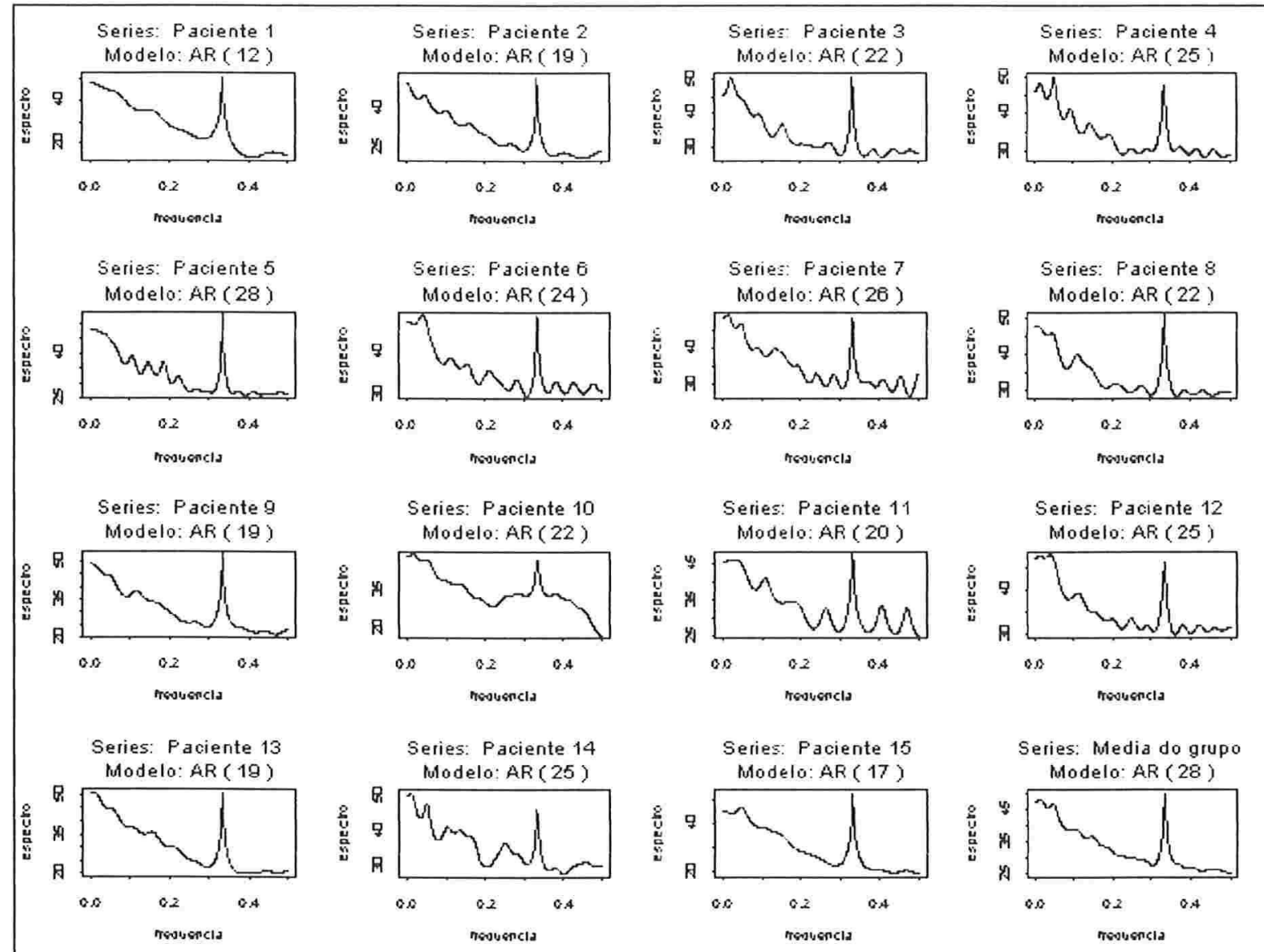

Figura 5.13: Estimador Espectral Auto-regressivo do eletroencefalograma da região F3 (Frontal - esquerda) de 15 pacientes com sinal alterado 


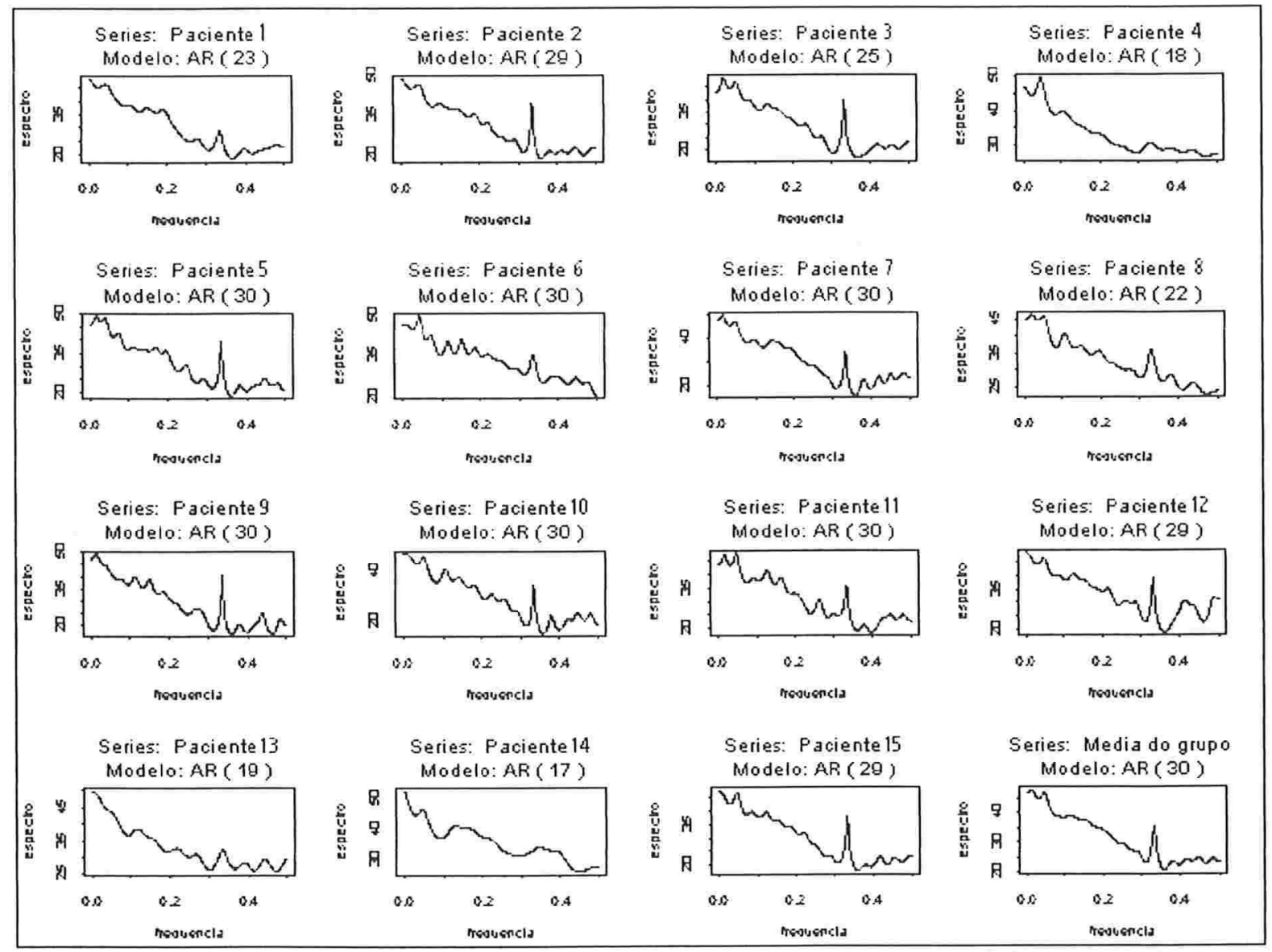

Figura 5.3: Estimador Espectral Auto-regressivo do eletroencefalograma da região F4 (Frontal - direita) de 15 pacientes com sinal normal 


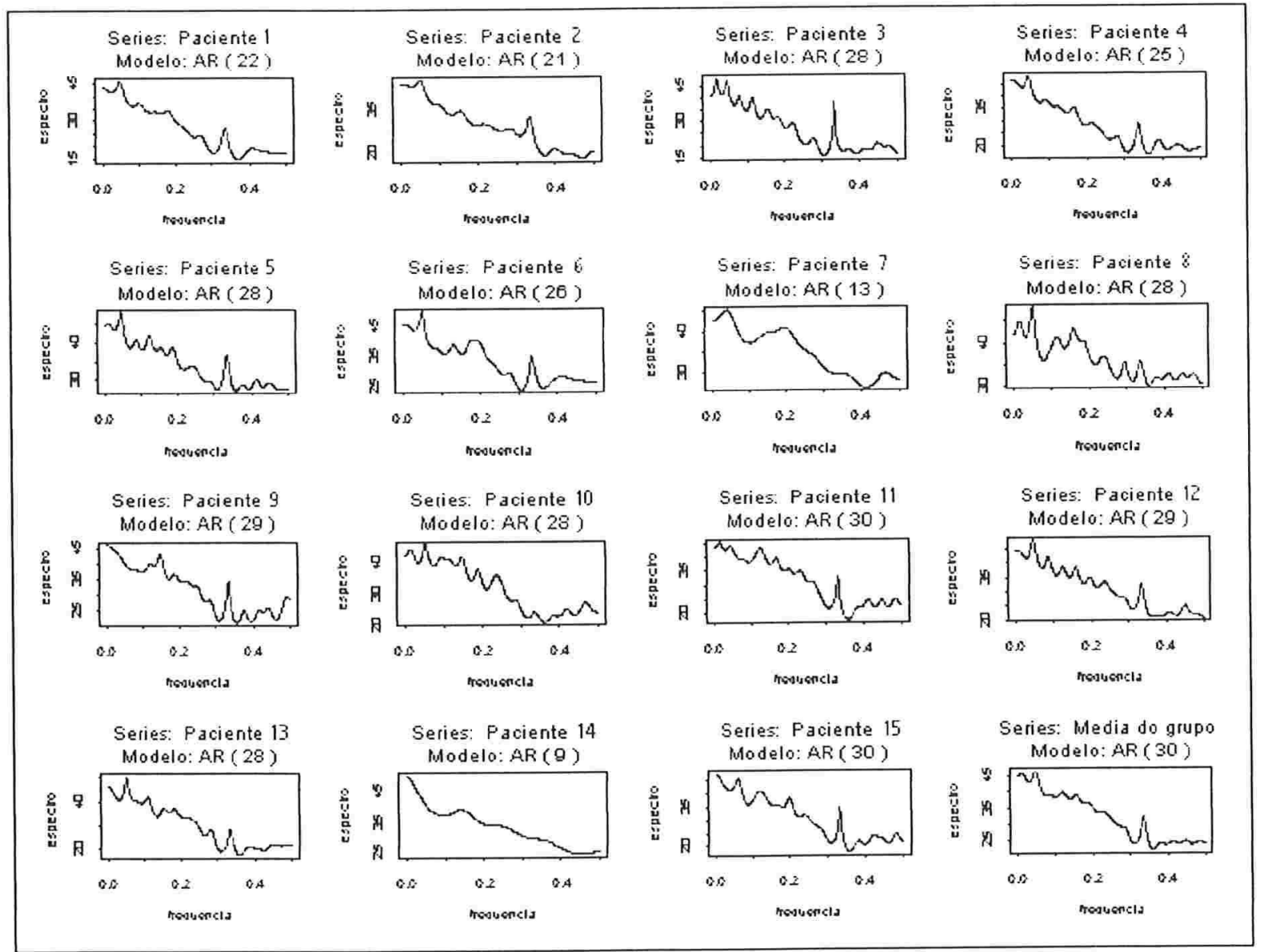

Figura 5.3: Estimador Espectral Auto-regressivo do eletroencefalograma da região T3 (Temporal - esquerda) de 15 pacientes com sinal alterado 


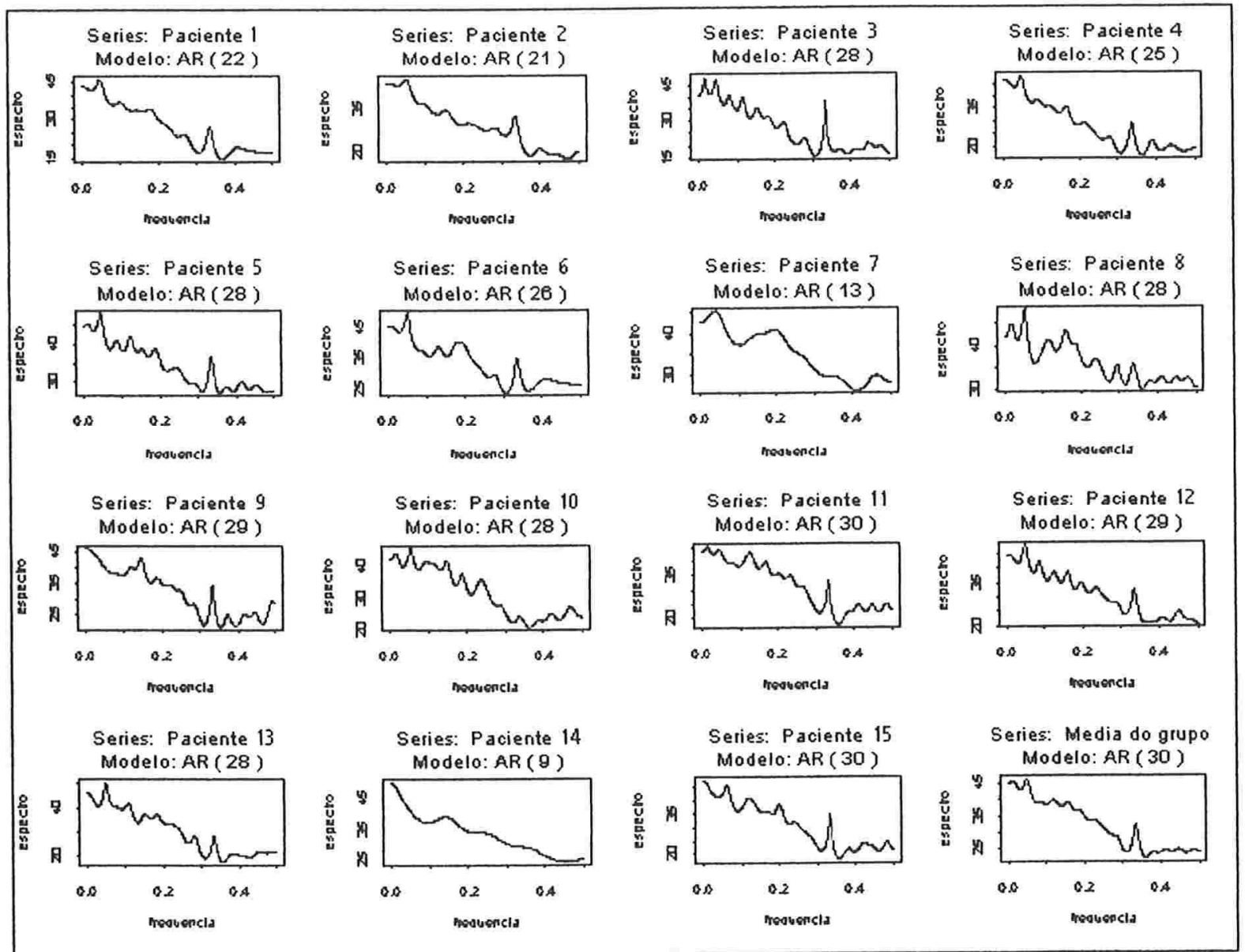

Figura 5.3: Estimador Espectral Auto-regressivo do eletroencefalograma da região T4 (Temporal - direita) de 15 pacientes com sinal normal

Utilizando-se (3.14) calculamos $\hat{h}_{i}(\mathbf{X})$. As Tabelas 5.4 a 5.7, representam as matrizes de confusão para o procedimento baseado-se no periodograma e no periodograma suavizado utilizando a janela de Daniell (ou retangular) com $L=10$, 50 e 100, respectivamente. Apresentamos, ainda, na Tabela 5.8 o procedimento utilizando o estimador espectral auto-regressivo. 
Tabela 5.4:Matriz de confusão para o modelo discriminante - Periodograma

\begin{tabular}{|c|c|c|c|c|c|}
\hline \multicolumn{2}{|c|}{} & \multicolumn{4}{|c|}{ Classificação Prevista } \\
\cline { 2 - 6 } \multicolumn{2}{|c|}{} & F3 & F4 & T3 & T4 \\
\hline \multirow{3}{*}{$\begin{array}{c}\text { Classificação } \\
\text { Real }\end{array}$} & F3 & 15 & 0 & 0 & 0 \\
\cline { 2 - 6 } & F4 & 0 & 15 & 0 & 0 \\
\cline { 2 - 6 } & T3 & 0 & 0 & 15 & 0 \\
\cline { 2 - 6 } & T4 & 0 & 3 & 0 & 12 \\
\hline
\end{tabular}

Tabela 5.5:Matriz de confusão para o modelo discriminante - Periodograma Suavizado $(L=10)$

\begin{tabular}{|c|c|c|c|c|c|}
\hline \multicolumn{2}{|c|}{} & \multicolumn{5}{|c|}{ Classificação Prevista } \\
\cline { 3 - 6 } & F3 & F4 & T3 & T4 \\
\hline \multirow{3}{*}{$\begin{array}{c}\text { Classificação } \\
\text { Real }\end{array}$} & F3 & 13 & 2 & 0 & 0 \\
\cline { 2 - 6 } & T3 & 0 & 15 & 0 & 0 \\
\cline { 2 - 6 } & T4 & 0 & 2 & 13 & 0 \\
\hline
\end{tabular}

Tabela 5.6: Matriz de confusão para o modelo discriminante - Periodograma Suavizado $(\mathrm{L}=50)$

\begin{tabular}{|c|c|c|c|c|c|}
\hline \multicolumn{2}{|c|}{} & \multicolumn{4}{|c|}{ Classificação Prevista } \\
\cline { 2 - 6 } \multicolumn{2}{|c|}{} & F3 & F4 & T3 & T4 \\
\hline \multirow{3}{*}{$\begin{array}{c}\text { Classificação } \\
\text { Real }\end{array}$} & F3 & 15 & 0 & 0 & 0 \\
\cline { 2 - 6 } & F4 & 0 & 15 & 0 & 0 \\
\cline { 2 - 6 } & T3 & 0 & 1 & 14 & 0 \\
\cline { 2 - 6 } & T4 & 0 & 4 & 0 & 11 \\
\hline
\end{tabular}


Tabela 5.7:Matriz de confusão para o modelo discriminante - Periodograma Suavizado ( $L=100$ )

\begin{tabular}{|c|c|c|c|c|c|}
\hline \multicolumn{2}{|c|}{} & \multicolumn{5}{|c|}{ Classificação Prevista } \\
\cline { 2 - 6 } \multicolumn{2}{|c|}{} & F3 & F4 & T3 & T4 \\
\hline \multirow{3}{*}{$\begin{array}{c}\text { Classificação } \\
\text { Real }\end{array}$} & F3 & 15 & 0 & 0 & 0 \\
\cline { 2 - 6 } & F4 & 3 & 12 & 0 & 0 \\
\cline { 2 - 6 } & T3 & 0 & 2 & 13 & 0 \\
\cline { 2 - 6 } & T4 & 0 & 4 & 1 & 10 \\
\hline
\end{tabular}

Tabela 5.8:Matriz de confusão para o modelo discriminante - Estimador Espectral Auto-regressivo

\begin{tabular}{|c|c|c|c|c|c|}
\hline \multicolumn{2}{|c|}{} & \multicolumn{5}{|c|}{ Classificação Prevista } \\
\cline { 3 - 6 } \multicolumn{1}{|c|}{} & F3 & F4 & T3 & T4 \\
\hline \multirow{3}{*}{$\begin{array}{c}\text { Classificação } \\
\text { Real }\end{array}$} & F3 & $\mathbf{5}$ & 10 & 0 & 0 \\
\cline { 2 - 6 } & F4 & 0 & 11 & 3 & 1 \\
\cline { 2 - 6 } & T3 & 0 & 3 & 12 & 0 \\
\cline { 2 - 6 } & T4 & 0 & 5 & 5 & 5 \\
\hline
\end{tabular}




\subsection{Conclusões}

Observando-se os resultados obtidos, notamos que os métodos paramétricos (análise no domínio do tempo e estimador espectral auto-regressivo) apresentaram resultados semelhantes (TAE $=0,45)$. Tal inadequabilidade destes métodos espelha, de certa forma, a dificuldade dos modelos auto-regressivos em ajustar séries complexas com múltiplas periodicidades. Entre os demais estimadores, verificamos que o estimador não suavizado apresentou a melhor performance $(\mathrm{TAE}=0,05)$. Dentre os estimadores suavizados, o melhor desempenho foi o obtido utilizando-se uma janela espectral de tamanho 50 (TAE= 0,$13 ; 0,08 ; 0,17$ para $L=10,50$ e 100, respectivamente). Além disso, se observarmos os tipos de erros cometidos, notaremos que no estimador não suavizado, estes correspondem a séries pertencentes a região T4, classificados como pertencentes a F4, entretanto, ambos normais. Desta forma, podemos concluir que, em séries mais simples, ou seja, aquelas na qual podemos ajustar modelos do tipo $\operatorname{ARMA}(p, q)$ e que não possuam várias periodicidades, o método de discriminação no domínio do tempo apresenta resultados muito superiores aos obtidos pelas técnicas no domínio da frequência, chegando em alguns casos ser superior em até 50\%. Entretanto, quando nos deparamos com séries com múltiplas periodicidades, os resultados invertem-se, sendo o estimador não suavizado o melhor método. A utilização dos estimadores espectrais autoregressivos, apesar de suas boas propriedades, no caso específico do problema de discriminação, não apresenta vantagens, visto apresentar os mesmos problemas da técnica no domínio do tempo e possuir o grau de acerto semelhante às técnicas não paramétricas no caso de modelos simples. A verificação deste desempenho no caso de outros tipos de modelos e no caso multivariado, são tópicos que deverão ser abordados posteriormente. 


\section{Bibliografia}

1. Akaike, H. (1969). Fitting autoregressive model for prediction. Annals of the Institute of Statistical Mathematics, 21: 243-247.

2. Ara, A. B. (1982). Estimadores Espectrais Auto-Regressivos. Dissertação de Mestrado. Instituto de Matemática e Estatística-IME, São Paulo, Dept. de Estatística.

3. Bloomfield, P. (1976). Fourier Analysis of Time Series: an Introduction. New York: John Wiley \& Sons.

4. Box, G. E. P., Jenkins, G. M., Reinsel, G. C. (1994). Time Series Analysis Forecasting and Control. New Jersey: Prentice Hall.

5. Brillinger, D. R. (1981) Time Series, Data Analysis and Theory. San Francisco:Holden Day, Inc.

6. Brillinger, D. R. and Krishnaiah, P. R. (1983). Time Series in Frequency Domain. Netherlands: Elsevier Science Publishers B.V.

7. Johnson, R. A. and Wichern, D. (1982). Applied Multivariate Statistical Analysis. New Jersey: Prentice Hall.

8. Morettin, P. A. e Toloi, C. M. C. (1987). Previsão de Séries Temporais. 2a. ed. São Paulo: Atual.

9. Parzen, E. (1969). Some recent advances in time series modeling. IEEE Transactions on Automatic Control, 19 (6): 723-730.

10. Priestley, M. B. (1981). Spectral Analysis and Time Series. San Diego: Academic Press. 
11. Rayner, J. N. (1971). An Introduction to Spectral Analysis. London:Pion Limited.

12. Rocha, J. G. C. (1983). Testes para Componentes Periódicas em Séries Temporais. Dissertação de Mestrado. Instituto de Matemática e Estatística-IME, São Paulo, Dept. de Estatística.

13. Shumway, R. H. (1988) Applied Statistical Time Series Analysis. Englewood Cliffs, N.J.: Prentice Hall.

14.Wei, W. W. S. (1990). Time Series Analysis. Addison-Wesley Publishing Company. 


\section{APÊNDICE A}

\section{Programas}

\section{A1- Análise Discriminante no Domínio do Tempo}

Disc.arima_function $(x, n, M, q)$

\{

\# ${ }^{\star \star \star *}$ Ajuste de modelos Autorregressivos para cada série ${ }^{* \star \star \star *}$ \#

\# Cálculo do número de séries evolvidas \#

n.series_sum(n)-n[[1]]

max.ordem_30

infinity_1e+30

parametro_vector("list",n[[1]])

\# Realizando ajuste para o primeiro grupo \#

M.fit_matrix(nrow=n[[2]], ncol=1)

for (i in 1:n[[2]]) M.fit[i]_ar( aic="T",order=max.ordem,M[,i] )\$order

\#Calculando o parâmetro para o modelo do 1o. grupo \#

\# Média dos parâmetros de cada série componente do grupo\#

parametro[[1]]\$ordem_max(M.fit)

parametro[[1]]\$coef_matrix $(0$, nrow= parametro[[1]]\$ $\$$ ordem, ncol=1)

lixo_matrix(nrow=max(M.fit), ncol=n[[2]]+1)

win.graph() 


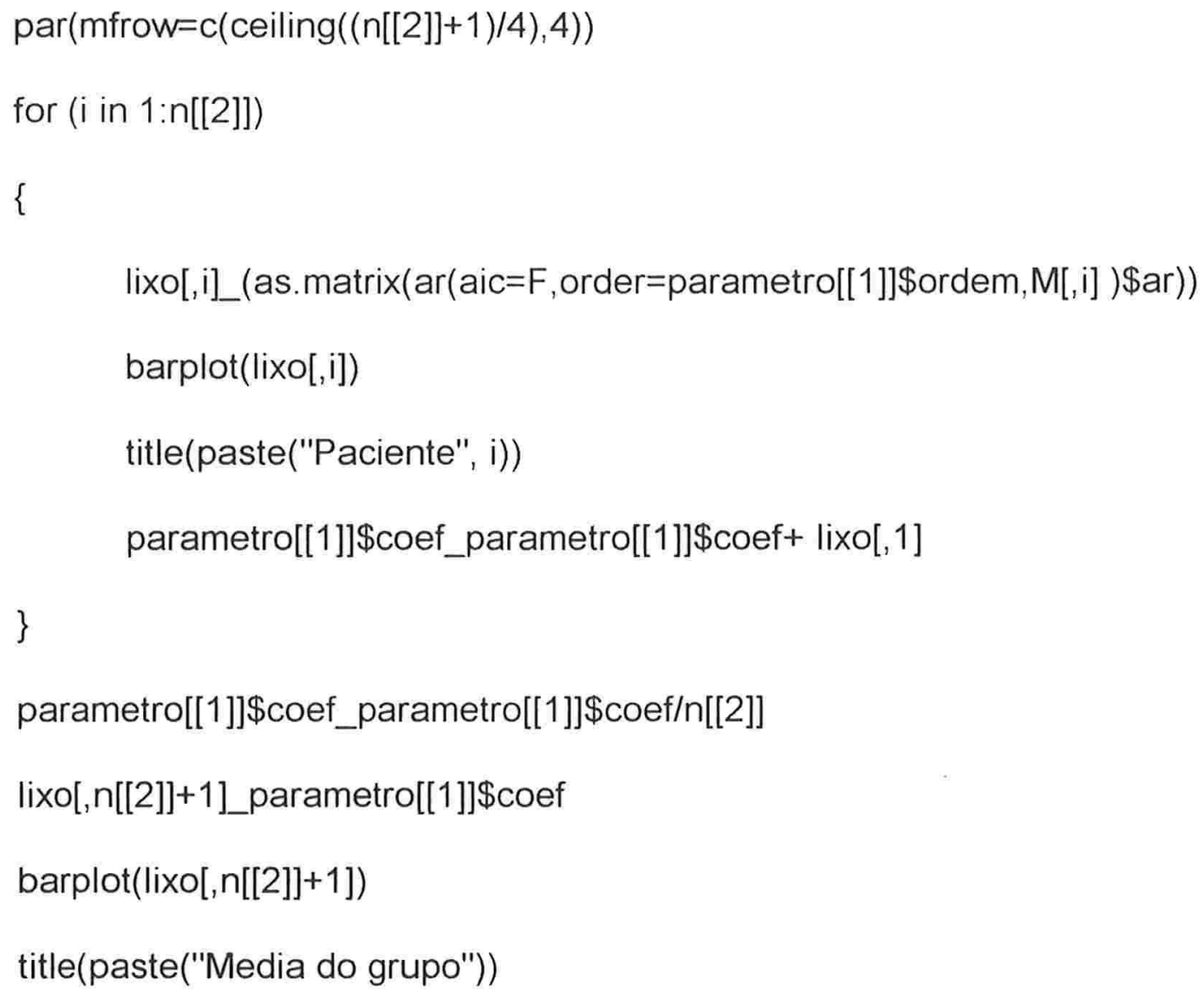




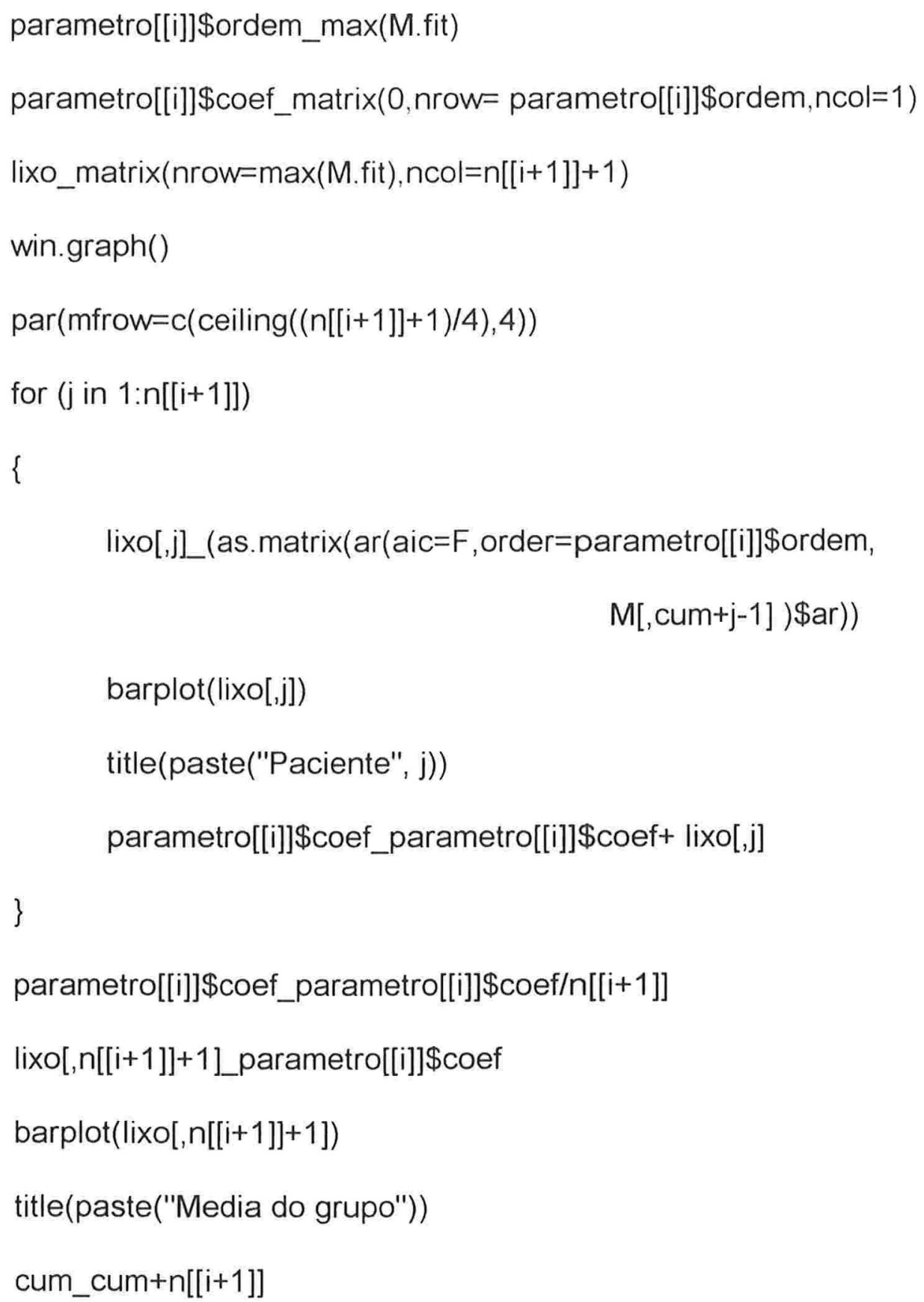


for $(i$ in $1: n \operatorname{col}(x))$

\{

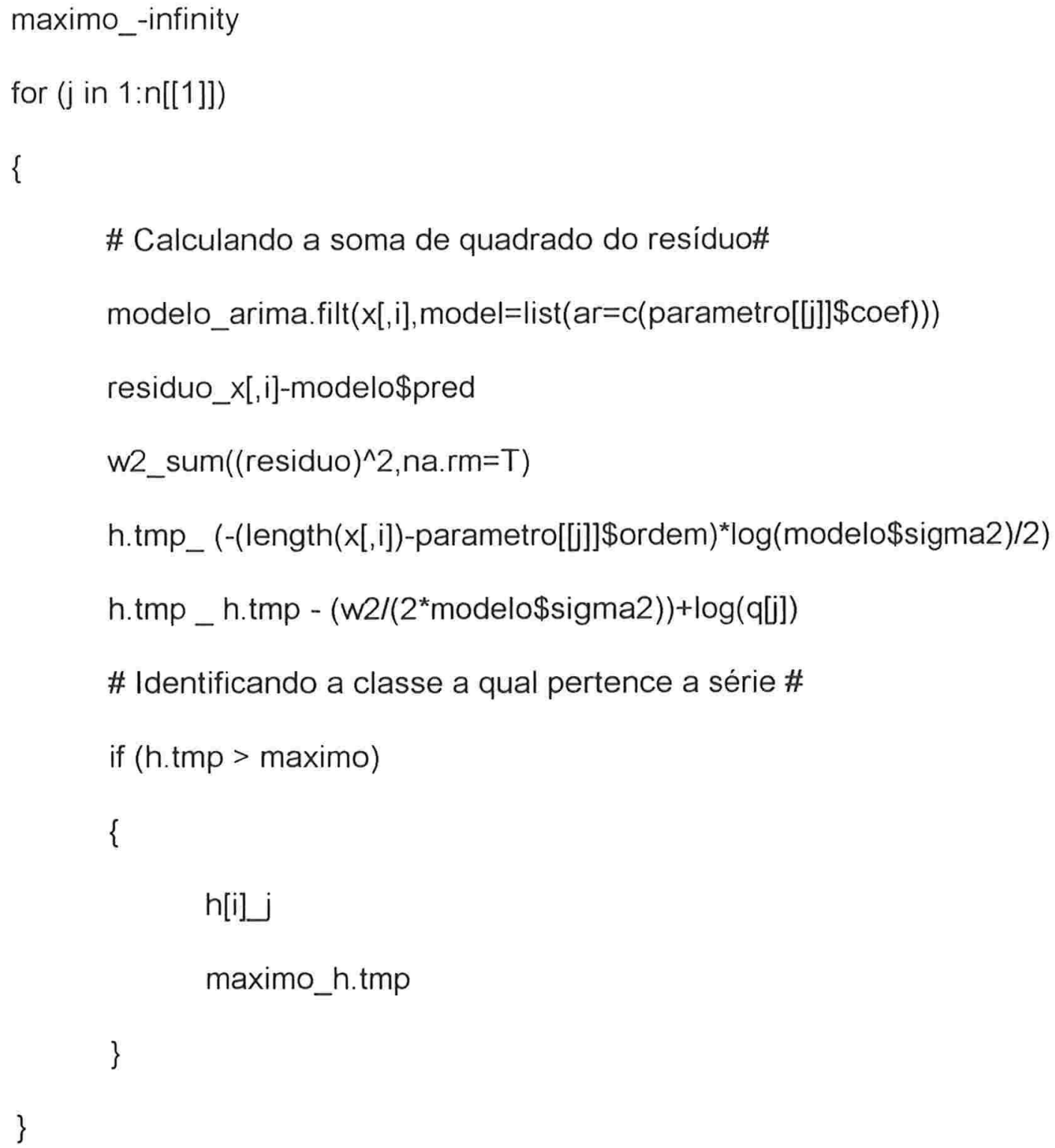


A2- Análise Discriminante no Domínio da Frequência -

Periodograma/Periodograma Suavizado

Disc.spc_function(x, n,M,q, span1)

\{

$\#^{* \star \star \star}$ Cálculo da Transformada de Fourier para cada série ${ }^{\star \star \star \star \star}$ \#

\# Cálculo do número de séries evolvidas \#

n.series_sum(n)-n[[1]]

max.ordem_30

infinity_1e+30

\# Inicializando Matrix p/ armazenar as Transf. de Fourier Médias de cada grupo\#

if (exists("dft")) rm(dft)

dft_matrix $(0$, nrowenrow(M),ncol=n[[1]])

if (exists("per")) rm(per)

per_matrix $(0, \operatorname{nrow}=\operatorname{nrow}(\mathrm{M}), \mathrm{ncol}=\mathrm{n}[[1]])$

\# Inicializando contador \#

cum_1

\# Realizando calculo da DFT para cada grupo \#

for (i in $1: n[[1]])$

\{

win.graph()

$\operatorname{par}(\operatorname{mfrow}=c(\operatorname{ceiling}((n[[i+1]]+1) / 4), 4))$

\#Periodograma e Transformada Média para os grupos \# 


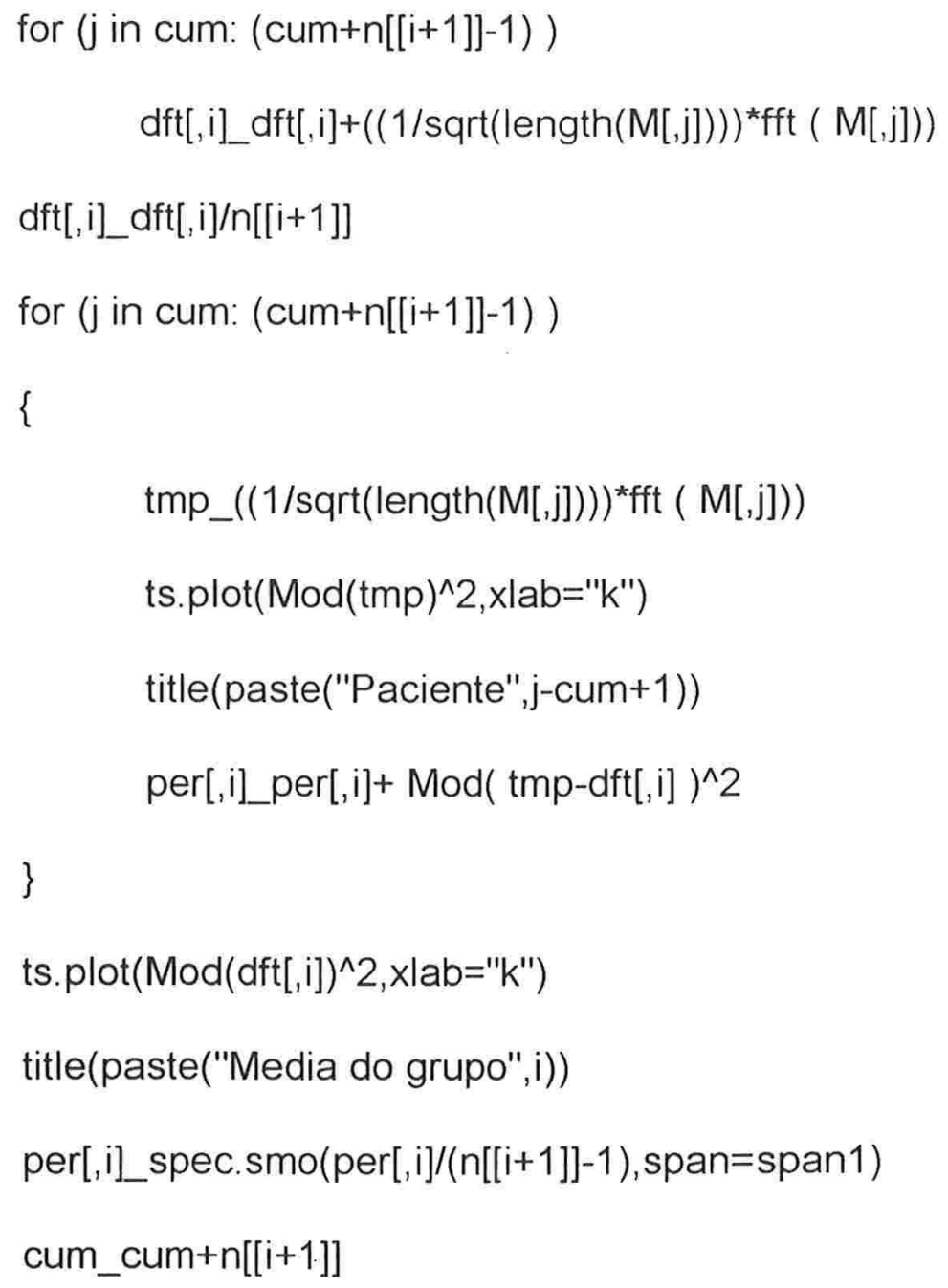


\{

\# Calculando a soma de quadrado do resíduo\#

h.tmp_(-1/2)*sum((Mod( ((1/sqrt(length $\left.(x[, i])))^{*} \mathrm{fft}(\mathrm{x}[, \mathrm{i}])\right)-$

$\left.\left.\operatorname{dft}[, j])^{\wedge} 2\right) / \operatorname{per}[, j]\right)$

h.tmp_h.tmp - (1/2)*sum(log(per[,j]))+log(q[j])

\# Identificando a classe a qual pertence a série \#

if ( $h . t m p>$ maximo)

\{

$h[i]$

maximo_h.tmp

\}

\}

\}

\# Construindo saída \#

h

\} 
A3- Análise Discriminante no Dominio da Frequência - Estimador Espectral Auto-regressivo

Disc.spcar_function(x, n,M,q)

\{

$\#^{\star \star \star \star \star}$ Ajuste de modelos Autorregressivos para cada série ${ }^{\star \star \star \star \star} \#$

\# Cálculo do número de séries evolvidas \#

n.series_sum(n)-n[[1]]

max.ordem_30

infinity_1e+30

parametro_vector("list",n[[1]])

\# Realizando ajuste para o primeiro grupo \#

M.fit_matrix(nrow=n[[2]],ncol=1)

win.graph()

$\operatorname{par}(\operatorname{mfrow}=c($ ceiling $((\mathrm{n}[[2]]+1) / 4), 4))$

for (i in $1: n[[2]])$

\{

tmp_ar( aic="T",order=max.ordem, M[,i] )

M.fit[i]_tmp\$order

spec.ar2(tmp, plot=T,k=i)

\}

\#Calculando o parâmetro para o modelo do 10. grupo \#

\# Média dos parâmetros de cada série componente do grupo\# 
parametro[[1]]\$ordem_max(M.fit)

for (i in $1: n[[2]])$

\{

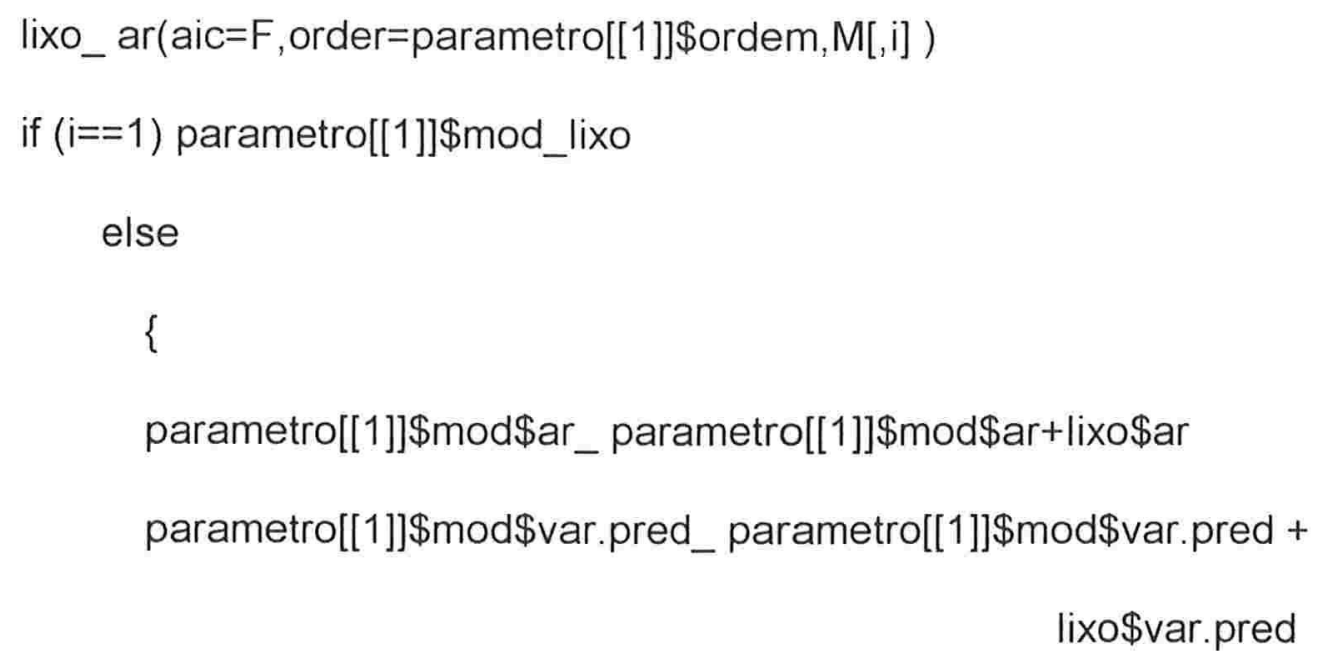

\# Inicializando contador \#

cum_n[[2]]+1

\# Realizando ajuste para os demais grupos e calculando funções discriminantes \# for (i in $2: n[[1]])$

\# Realizando ajuste para o demais grupos \#

M.fit_matrix(nrow=n[[i+1]],ncol=1)

win.graph() 


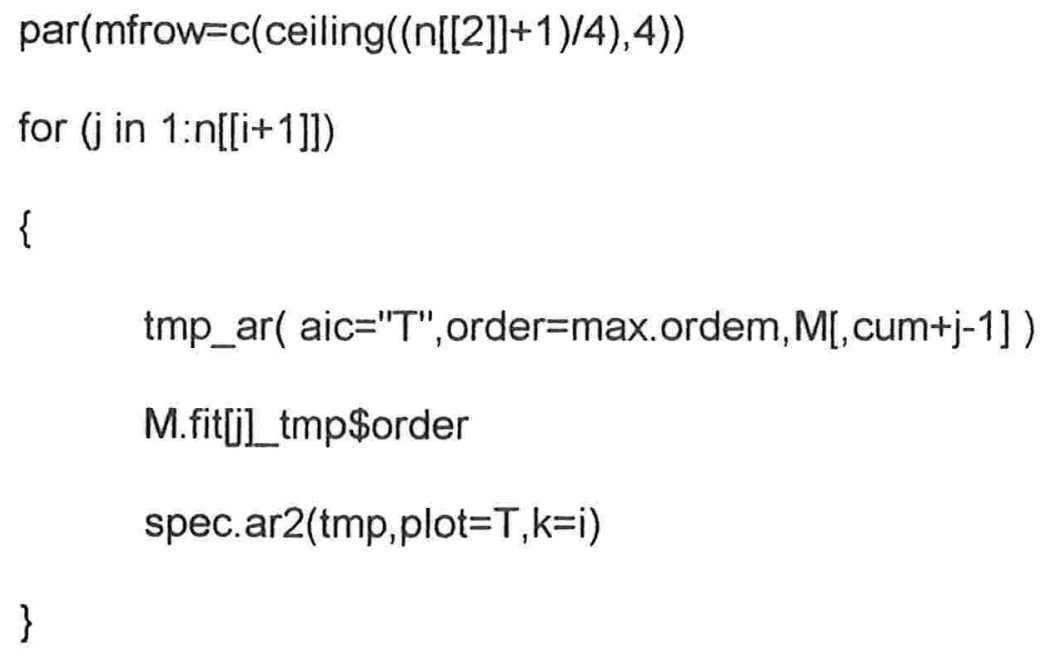


cum_cum $+n[[i+1]]$

\}

\# ${ }^{* \star * *}$ Cálculo da Transformada de Fourier para cada série ***** \#

\# Inicializando Matrix p/ armazenar as Transf. de Fourier Médias de cada grupo\#

if (exists("dft")) rm(dft)

dft_matrix $(0$, nrow=nrow $(M), n c o l=n[[1]])$

if (exists("per")) rm(per)

per_matrix $(0, \operatorname{nrow}=\operatorname{nrow}(\mathrm{M}), \mathrm{ncol}=\mathrm{n}[[1]])$

\# Inicializando contador \#

cum_1

\# Realizando calculo da DFT para cada grupo \#

for (i in 1:n[[1]])

\{

for ( $j$ in cum: (cum $+n[[i+1]]-1))$

$\left.\operatorname{dft}[, i] \_d f t[, i]+((1 / \text { sqrt(length }(M[, j])))^{\star} f f t(M[, j])\right)$

$\operatorname{dft}[, i] \_d f t[, i] / n[[i+1]]$

cum_cum $+n[[i+1]]$

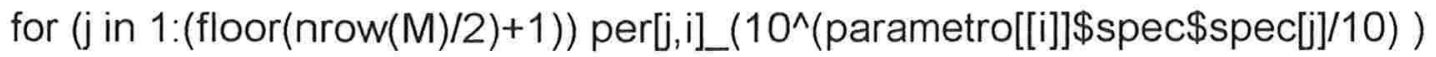

for $(j$ in 1: floor(nrow $(M) / 2)) \operatorname{per}[(\operatorname{nrow}(M)-j+1), i] \_\operatorname{per}[j, i]$

\} 
$\#^{\star \star \star \star *}$ Discriminação das séries contidas em $x^{\star \star \star \star \star *} \#$

\# Inicializando Matrix que armazenará os resultados \#

if (exists("h")) rm(h)

h_ matrix $(\operatorname{nrow}=\operatorname{ncol}(x), \operatorname{ncol}=1)$

for (i in 1:ncol(x))

\{

maximo_-infinity

for $(j$ in $1: n[[1]])$

\{

\# Calculando a soma de quadrado do resíduo\#

h.tmp_(-1/2)*sum((Mod( ((1/sqrt(length $\left.(x[, i])))^{*} f f t(x[, i])\right)-$

$\left.\left.\operatorname{dft}[, j])^{\wedge} 2\right) / \operatorname{per}[1: \operatorname{nrow}(M), j]\right)$

h.tmp _ h.tmp - (1/2)* sum(log(per[,j]))+log(q[j])

\# Identificando a classe a qual pertence a série \#

if (h.tmp > maximo)

\{

$h[i] \mathrm{j}$

maximo_h.tmp

\}

\}

\}

$\mathrm{h}$

\} 